\title{
LamaH | Large-Sample Data for Hydrology: Big data für die Hydrologie und Umweltwissenschaften
}

\author{
Christoph Klingler · Karsten Schulz · Mathew Herrnegger
}

\author{
Angenommen: 15. April 2021 / Online publiziert: 19. Mai 2021
}

(C) Der/die Autor(en) 2021

\begin{abstract}
Zusammenfassung „Big data“ ist zwar ein inflationär verwendetes Modewort, aber sehr umfangreiche Datensätze finden auch im Bereich der Hydrologie zunehmend Verwendung. Anhand „large-
\end{abstract}

\section{Anmerkungen Der vorliegende Artikel ist eine verkürzte Übersetzung des englischen Beitrags „LamaH | Large-Sample Data for Hydrology and Environmental Sciences for Central Europe“, welcher beim Journal ESSD eingereicht worden ist (Klingler et al. 2021). Dieses Projekt wurde zum Teil vom Österreichischen Fonds zur Förderung der wissenschaftlichen Forschung (FWF) finanziert (Projektnummer P 31213). Die gewässerkundlichen Dienste Bayern sowie Baden-Württemberg überprüfen rückwirkend die Abflussdaten und adaptieren diese auch gegebenenfalls. Es kann daher sinnvoll sein, aktuellere Abflussdaten von GKD (2020) bzw. LUBW (2020) zu beziehen. Der Haftungsausschluss (Disclaimer) bei Zenodo ist ebenfalls zu beachten.}

\footnotetext{
Datenverfügbarkeit LamaH kann kostenlos unter https:/ / doi.org/10.5281/zenodo. 4525244 bezogen werden. Der Datensatz ist grundsätzlich mit Einzugsgebietsabgrenzung A/B/C, Pegel, Gewässernetz, hydrol. Modell sowie Appendix in 7 Bereiche gegliedert. Die ersten 4 Bereiche beinhalten neben den verschiedenen Textfiles bezüglich der Attribute sowie Zeitreihen auch Shapefiles. Die Abbildung des Gewässernetzes erfolgt anhand von Shapefiles, welche zahlreiche Attribute beinhalten. Für das hydrologische Modell (Klingler et al. 2021) werden verschiedene Einund Ausgaben (z.B. Zeitreihen oder Auswertungen) bereitgestellt. Die gesamte Ordnerstruktur, ergänzende Informationen hinsichtlich der Zeitreihen sowie die notwendigen zusätzlichen Referenzen bei Verwendung von LamaH sind im Ordner „Info“ enthalten.
}

sample“ (große Stichprobe)-Untersuchungen können Einblicke in hydrologische Prozesse und den hydrologischen Kreislauf gewonnen werden, welche bei kleinräumigen Studien eventuell verwehrt bleiben würden. LamaH (Large-Sample Data for Hydrology) ist ein neuer Datensatz für "large-sample“ Untersuchungen im Zentrum von Europa. Er deckt das gesamte orographische Einzugsgebiet der oberen Donau bis zur Staatsgrenze Österreich/Slowakei sowie alle anderen Einzugsgebiete in Österreich inkl. deren Oberlieger in benachbarten Ländern ab. LamaH umfasst somit eine Fläche von rund $170.000 \mathrm{~km}^{2}$ in 9 verschiedenen Ländern und erstreckt sich dabei von Flachlandregionen mit kontinental geprägtem Klima bis hin zu hochalpinen Zonen mit ganzjähriger Schnee- und Eisbedeckung. Folglich ist eine große Diversität an verschiedenen Eigenschaften in den 859 beobachteten Einzugsgebieten vorhan-

Erforderliche zusätzliche Referenzen bei Verwendung von LamaH Wir ersuchen höflich um Beachtung, da die Zustimmung zum Zitieren der Primärquelle meist die Voraussetzung für die Weitergabe der Daten ist: BAFU (2020); CHMI (2020); GKD (2020); HZB (2020); LUBW (2020); BMLFUW (2013); Broxton et al. (2014); CORINE (2012); EEA (2019); ESDB (2004); Farr et al. (2007); Friedl und Sulla-Menashe (2019); Gleeson et al. (2014); HAO (2007); Hartmann und Moosdorf (2012); Hiederer (2013a, b); Linke et al. (2019); Muñoz Sabater et al. (2021); Muñoz Sabater (2019a); Myneni et al. (2015); Pelletier et al. (2016); Toth et al. (2017); Trabucco und Zomer (2019); Vermote (2015).

DI C. Klingler $(\varangle) \cdot$

Univ.-Prof. Dipl.-Geoökol. Dr. K. Schulz .

DI Dr. M. Herrnegger

Institut für Hydrologie

und Wasserwirtschaft,

Universität für Bodenkultur,

Muthgasse 18, 1190 Wien, Österreich

christoph.klingler@boku.ac.at den, welche anhand von über 60 verschiedenen statischen Attributen in den Kategorien Topographie, Klimatologie, Hydrologie, Landbedeckung, Vegetation, Boden sowie Geologie erfasst wird. LamaH umfasst zudem eine Sammlung an Abflusszeitreihen sowie verschiedenste meteorologische Zeitreihen. Alle Zeitreihen liegen in täglicher und stündlicher Auflösung vor, während alle meteorologischen sowie ein Großteil der Abflusszeitreihen eine Spanne von über 35 Jahren aufweisen. Die Abflusspegel sind ferner mit über 20 verschiedenen Attributen klassifiziert, wobei auch anthropogene Pegelbeeinflussungen sowie Indikatoren für Datenqualität und -vollständigkeit beschrieben werden. Die Struktur von LamaH ist grundsätzlich an jene der CAMELS-Datensätze angelehnt. Im Gegensatz dazu werden bei LamaH Daten jedoch nicht nur für unabhängige und damit teilweise sehr große Einzugsgebiete bereitgestellt, sondern auch für kleinere Zwischeneinzugsgebiete. Erstmals kann dadurch bei hydrologischen „large-sample“-Datensätzen ein großes zusammenhängendes hydrologisches Netzwerk zur Verfügung gestellt werden. Neben den Datengrundlagen, der Methodik der Datenaufbereitung und der räumlichen Verteilung ausgewählter Einzugsgebietseigenschaften werden auch etwaige Unsicherheiten bzw. Limitierungen diskutiert. Des Weiteren sind auch mögliche Anwendungsmöglichkeiten angeführt, schließlich sollte LamaH vor allem als Grundlage für weiterführende Untersuchungen dienen. LamaH kann kosten- und barrierefrei unter https://doi.org/10.5281/zenodo. 4525244 bezogen werden.

Schlüsselwörter Large-sample Hydrologie · Datensatz . Zentraleuropa $\cdot$ Einzugsgebietshydrologie - Abflusszeitreihen . Meteorologische Zeitreihen · Einzugsgebietseigenschaften · ERA5Land - CAMELS 
LamaH | Large-Sample Data for Hydrology: big data for hydrology and environmental sciences

Abstract Very large and comprehensive datasets are increasingly used in the field of hydrology. Large-sample studies provide insights into the hydrological cycle that might not be available with small-scale studies. LamaH (Large-Sample Data for Hydrology) is a new dataset for large-sample studies and comparative hydrology in Central Europe. It covers the entire catchment of the upper Danube to the state border Austria/Slovakia, as well as all other Austrian catchments including their foreign upstream areas. LamaH covers an area of about $170,000 \mathrm{~km}^{2}$ in 9 different countries, ranging from lowland regions characterized by a continental climate to high alpine zones dominated by snow and ice. Consequently, a wide diversity of properties is present in the individual catchments. We represent this variability in 859 gauged basins with over 60 static catchment attributes, covering topography, climatology, hydrology, land cover, vegetation, soil and geological properties. LamaH further contains a collection of runoff time series as well as meteorological time series. These time series are provided with daily and also hourly resolution. All meteorological and the majority of runoff time series cover a span of over 35 years, which enables long-term analyses, also with a high temporal resolution. The runoff time series are classified by over 20 different attributes including information about human impacts and indicators for data quality and completeness. The structure of LamaH is based on the well-known CAMELS datasets. In contrast, however, LamaH does not only consider independent basins, covering the full upstream area. Intermediate catchments are included as well, which allows, together with novel attributes, to consider the hydrological network and river topology in applications. We discuss not only the data basis and the methodology of data preparation, but also focus on possible limitations and uncertainties. Potential applications of LamaH are outlined as well, since it is intended to serve as a solid basis for further research. LamaH is available at https://doi.org/10.5281/zenodo. 4525244
Keywords Large-sample hydrology · Large-sample dataset . Central Europe · Catchment hydrology · Runoff time series - Meteorological time series - Catchment attributes - ERA5Land $\cdot$ CAMELS

\section{Einleitung}

Die Hydrologie und hydrologische Prozesse sind durch eine hohe zeiträumliche Variabilität gekennzeichnet. Bei der Abflussgenerierung in kleinräumigen, alpinen Einzugsgebieten mit steiler und komplexer Topographie dominieren andere Prozesse als bei Tieflandflüssen mit flacher Topographie. Der Wasserhaushalt in einem energielimitierten, humiden Einzugsgebiet in Europa gestaltet sich gänzlich anders als beispielsweise in einem wasserlimitierten Einzugsgebiet in trockenen (semi-) ariden Gebieten in Afrika oder Australien. Ein Wassertropfen, der über die russische Lena in das Arktische Meer fließt, hat eine gänzlich andere Biografie als ein Wassertropfen aus Ruanda in Zentralafrika, der nach über $6600 \mathrm{~km}$ über den Nil in das Mittelmeer mündet. Randbedingung und wesentliche Treiber für die Unterschiede sind die Einzugsgebietseigenschaften, die sich über topographische und hydroklimatische Attribute, Landbedeckung, Geologie und die Bodenverhältnisse beschreiben lassen.

Zur Vertiefung des Prozessverständnisses und damit auch für die Verbesserung der Modelle sowie zur Steigerung des Vertrauens der Vorhersagen für die Praxis ist es nötig, diese zeiträumliche Variabilität in unseren Überlegungen zu berücksichtigen. In den letzten Jahrzehnten gab es eine Reihe von internationalen Initiativen (z. B. Distributed Model Intercomparison Project - DMIP, Smith et al. 2004; Inter-Sectoral Impact Model Intercomparison Project ISI-MIP, Warszawski et al. 2014; Model Parameter Estimation Project-MOPEX, Duan et al. 2006; Hydrological Ensemble Prediction Experiment - HEPEX, Schaake et al. 2007), die das Ziel verfolgt haben, durch umfassende Modellvergleiche in unterschiedlichen Regionen der Welt Fortschritte in der Vorhersage von hydrologischen Variablen zu erzielen. Neuere Bestrebungen zielen darauf ab, homogene und konsistente Datensätze bereitzustellen, mit denen individuelle Personen und Arbeitsgruppen Modellentwicklungen und Modellvergleiche durchführen können. Dabei ist ein Trend zu immer umfangreicheren und vollständigeren Datensätzen erkennbar, der wie folgt begründet werden kann: 1) Mittels Fernerkundung ist es möglich geworden, die Beschaffenheit der Atmosphäre und Erdoberfläche einheitlich auf globaler Ebene zu erfassen. Einer breiteren Allgemeinheit sind vermutlich die Sentinel- (ESA 2021; Malenovsky et al. 2012), Landsat- (NASAa 2021; Irons et al. 2012) sowie MODISMissionen (NASAb 2021; Barnes et al. 2003) bekannt. 2) Auch neue Plattformen bzw. Applikationen zum Bezug und zur Verarbeitung dieser meist sehr datenlastigen Fernerkundungsprodukte erleichtern die Handhabung ungemein. $\mathrm{Zu}$ nennen sind hier unter anderem die Plattform „Google Earth Engine“ (GEEa 2021; GEEb 2021; Gorelik et al. 2017; Klingler et al. 2020), der „Copernicus Open Access Hub“ (COPa 2021) oder der „Copernicus Climate Data Store" (COPb 2021). 3) Parallel dazu gibt es zunehmenden Konsens darüber, dass das Verständnis über die mitunter äußerst komplexen Prozesse des hydrologischen Kreislaufes durch „largesample"-Untersuchungen vertieft werden kann (Gupta et al. 2014). „Largesample“-Hydrologie stützt sich dabei auf Informationen aus einer Vielzahl an unterschiedlichen Einzugsgebieten, um robuste Schlussfolgerungen ableiten $\mathrm{zu}$ können (Addor et al. 2019). Daher haben sich bereits mehrere Forschungsgruppen in verschiedenen Bereichen der Hydrologie mit „large-sample“-Studien beschäftigt (z.B. Berghuijs et al. 2014; Blöschl et al. 2019a; Döll et al. 2016; Gudmundsson et al. 2019; Luke et al. 2017; Kuentz et al. 2017; Singh et al. 2014; Van Lanen et al. 2013). 4) Schließlich haben datengetriebene Modelle oder "Machine Learning“Ansätze in jüngster Zeit große Aufmerksamkeit in der Hydrologie erlangt (Sit et al. 2020). Unabhängig davon, dass diese Entwicklungen kontrovers diskutiert werden (Nearing et al. 2020), basiert die Leistung bei der Zeitreihenvorhersage, auch in unbeobachteten Einzugsgebieten (z.B. Kratzert et al. 2019a), auf der Fähigkeit des maschinellen Lernens, Muster und $\mathrm{Zu}$ sammenhänge in Daten zu erkennen (Kratzert et al. 2019b). Diese „Machine Learning“-Ansätze sind jedoch stark von der Verfügbarkeit von Datensätzen mit einem großen Stichprobenumfang abhängig (z. B. Kratzert et al. 2019a, b, 2018). 


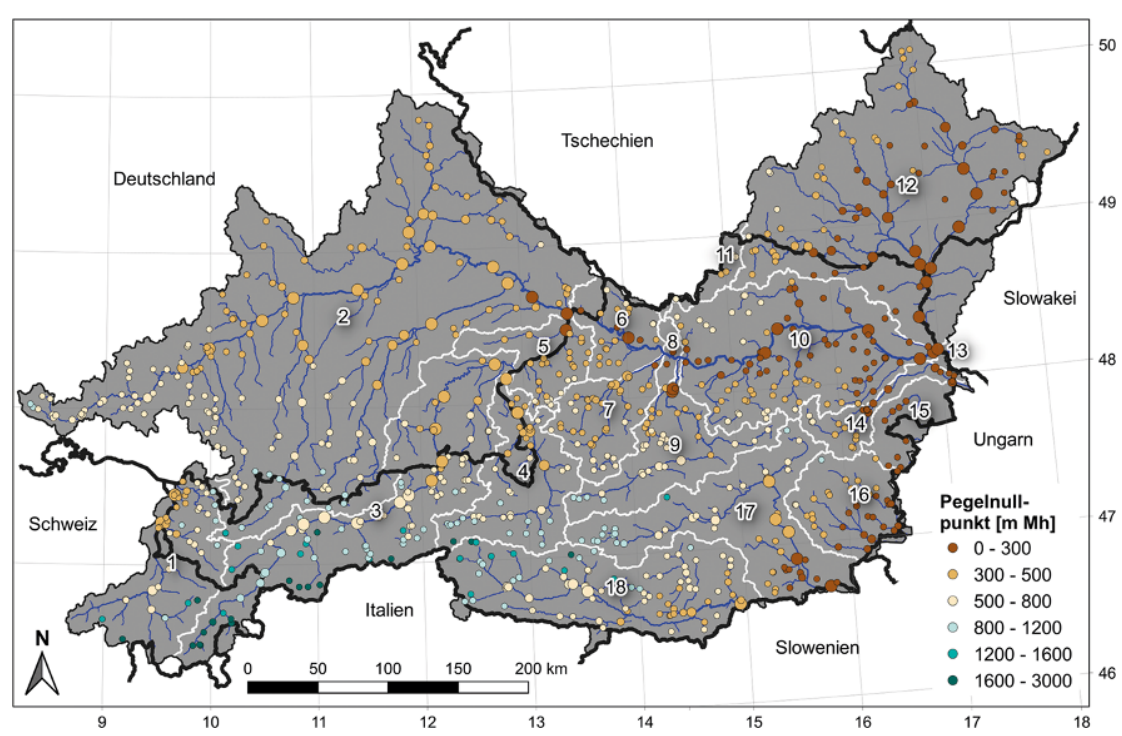

Abb. 1 Übersicht des in LamaH abgedeckten Gebiets (graue Farbe) sowie der Abfluss-Pegel (die Größe der Kreise ist proportional zur Einzugsgebietsfläche). Die Fläche kann in 18 verschiedene Flussgebiete unterteilt werden, welche durch die weißen Linien abgegrenzt sind. Die schwarzen Nummern sind dabei Abkürzungen für die einzelnen Flussgebiete, welche in Tab. 1 erläutert werden. Die Staatsgrenzen sind als dicke schwarze Linien dargestellt. Quelle des Gewässernetzes: HydroATLAS (Linke et al. 2019)

Aufgrund des mitunter sehr hohen Arbeitsaufwands und Umfangs von „large-sample“-Untersuchungen bietet es sich an, zwischen der Datenbasis sowie den darauf aufbauenden Untersuchungen $\mathrm{zu}$ differenzieren und diese getrennt zu publizieren. Dies eröffnet die Möglichkeit, die Datenbasis ausführlicher $\mathrm{zu}$ beschreiben und somit eine solide Grundlage für darauf aufbauende Studien zu schaffen. Eine Auswahl von bereits veröffentlichten „large-sample“-Datensätzen kann unter anderem der Tab. 1 in Gupta et al. (2014) entnommen werden. Weitere Datensätze für hydrologische Anwendungen sind unter anderem Global Runoff Reconstruction (Ghiggi et al. 2019), Global Streamflow Indices and Metadata Archive (Do et al. 2018; Gudmundsson et al. 2018), HydroATLAS (Linke et al. 2019), HydroSHEDS (Lehner et al. 2008) sowie die CAMELS (CAtchment Attributes and MEteorology for Large-sample Studies, Addor et al. 2017a) Kollektion. Die CAMELSDatensätze zeichnen sich durch eine konsistente Datenbearbeitung bzw. -struktur aus und es werden auch etwaige Limitierungen sowie Unsicherheiten diskutiert. Allerdings beinhaltet CAMELS nur Daten für einzelne, unabhängige Einzugsgebiete und nicht für ein zusammenhängendes Fluss- netzwerk (Addor et al. 2019). Der erste CAMELS-Datensatz wurde von Addor et al. (2017a) und Newman et al. (2015) für das zusammenhängende Territorium der Vereinigten Staaten veröffentlicht und deckt 671 Einzugsgebiete ab. Später folgten noch CAMELS-Datensätze für Chile (Alvarez-Garreton et al. 2018, 516 Einzugsgebiete), Brasilien (Chagas et al. 2020, 897 Einzugsgebiete) sowie Großbritannien (Coxon et al. 2020, 671 Einzugsgebiete). CAMELSDatensätze stellen dabei immer einen Verbund aus Abflusszeitreihen sowie auf das Pegeleinzugsgebiet aggregierte meteorologische Zeitreihen und statische Attribute dar.

LamaH (Large-Sample Data for Hydrology) ist ein neuer Datensatz für „large-sample“-Untersuchungen $\quad(859$ beobachtete Einzugsgebiete) in Zentraleuropa. Dieser beinhaltet neben Abflusszeitreihen auch meteorologische Zeitreihen sowie statische Einzugsgebietseigenschaften und basiert im Wesentlichen auf der Struktur der CAMELS-Datensätze. LamaH bietet darüber hinaus aber auch einige Neuerungen. So wird neben der bei den CAMELS-Datensätzen üblichen Einzugsgebietsabgrenzung, welche dem orographischen Einzugsgebiet der Pegel entspricht, bei LamaH auch eine Einzugsgebietsabgrenzung implementiert, welche die Zwischeneinzugsgebietsfläche (Differenzfläche) von benachbarten Pegeln abbildet. Mit zusätzlichen Attributen wie der Pegel-Topologie sowie der Fließlänge und dem Gefälle zwischen zwei angrenzenden Pegeln entsteht dabei eine Informationsbasis, welche das zusammenhängende hydrologische Netzwerk bzw. die Gewässertopologie spezifiziert. Dies eröffnet beispielsweise die Möglichkeit, die lokale Abflussbildung im Zwischeneinzugsgebiet getrennt vom Flussrouting zu modellieren. Eine weitere Neuerung ist die Auflösung der hydrometeorologischen Zeitreihen, welche bei LamaH im Gegensatz zu den CAMELS-Datensätzen neben der täglichen auch mit stündlicher Auflösung bereitgestellt werden. Zeitreihen mit stündlicher Auflösung sind beispielsweise entscheidend für ein zuverlässiges Ergebnis bei der Modellierung des Flussroutings oder von schnee- oder gletscherbeeinflussten Prozessen, welche ein systematisches Tagesmuster zeigen können.

Nach einer Beschreibung des Projektgebiets (Abschn. 2) sowie der verwendeten Einzugsgebietsabgrenzungen (Abschn. 3) wird die Aufbereitung der hydrometeorologischen Zeitreihen im Abschn. 4 erläutert. Abschn. 5 befasst sich mit statischen Einzugsgebietseigenschaften und zeigt deren räumliche Verteilung. Des Weiteren wird dort auch auf etwaige Unsicherheiten, Limitierungen sowie Einschränkungen der verwendeten Datenquellen hingewiesen. Zuletzt erfolgt im Abschn. 6 eine Zusammenfassung sowie eine Auflistung möglicher Anwendungsbereiche vom „large-sample“-Datensatz LamaH, der mitunter auch für Anwender aus anderen Bereichen der Umweltwissenschaften interessant sein könnte.

\section{Räumliche Ausdehnung}

LamaH umfasst eine Fläche von rund $170.000 \mathrm{~km}^{2}$ in 9 verschiedenen Ländern Zentraleuropas (Österreich, Deutschland, Tschechien, Schweiz, Slowakei, Italien, Liechtenstein, Slowenien und Ungarn; absteigend sortiert nach beitragender Fläche). Er deckt dabei die gesamte obere Donau bis zur Staatsgrenze Österreich/Slowakei sowie alle anderen Einzugsgebiete in Österreich inkl. deren Oberlieger in benachbarten Ländern ab. Den höchsten Punkt im abgedeckten Gebiet stellt der Piz Bernina mit $4049 \mathrm{~m}$ Mh dar, während der niedrigste Punkt mit ca. $130 \mathrm{~m} \mathrm{Mh}$ 
Tab. 1 Übersicht der 18 Flussgebiete

\begin{tabular}{|c|c|c|c|}
\hline $\mathrm{Nr}$ & Name des Flussgebietes & $\begin{array}{l}\text { Fläche in } \\
\text { LamaH } \\
{\left[\mathrm{km}^{2}\right]}\end{array}$ & Wichtige Quell- und Nebenflüsse \\
\hline 1 & Rhein & 7.610 & Vorderrhein, Hinterrhein, Plessur, Landquart, III, Frutz \\
\hline 2 & Donau oberhalb des Inns & 49.942 & $\begin{array}{l}\text { Iller, Brenz, Wörnitz, Lech, Altmühl, Naab, Regen, } \\
\text { Isar, IIz, Vils }\end{array}$ \\
\hline 3 & Inn oberhalb der Salzach & 15.249 & $\begin{array}{l}\text { Sanna, Ötztaler Ache, Sill, Ziller, Brandenberger Ache, } \\
\text { Brixentaler Ache, Mangfall, Alz }\end{array}$ \\
\hline 4 & Salzach & 6.684 & $\begin{array}{l}\text { Krimmler Ache, Fuscher Ache, Gasteiner Ache, Groß- } \\
\text { arlbach, Lammer, Saalach }\end{array}$ \\
\hline 5 & Inn unterhalb der Salzach & 4.060 & Mattig, Mühlheimer Ache, Rott, Pram \\
\hline 6 & Donau zwischen Inn und Traun & 2.870 & Erlau, Große Mühl, Innbach \\
\hline 7 & Traun & 3.851 & Ischl, Ager, Krems \\
\hline 8 & Donau zwischen Traun und Enns & 342 & Gusen \\
\hline 9 & Enns & 5.997 & Salza, Steyr \\
\hline 10 & $\begin{array}{l}\text { Donau zwischen Enns und } \\
\text { March }\end{array}$ & 15.492 & $\begin{array}{l}\text { Aist, Naarn, Ybbs, Erlauf, Melk, Pielach, Kamp, } \\
\text { Schwechat, Fischa }\end{array}$ \\
\hline 11 & Moldau & 763 & Maltsch \\
\hline 12 & March & 25.688 & Bečva, Thaya \\
\hline 13 & $\begin{array}{l}\text { Donau zwischen March und } \\
\text { Leitha }\end{array}$ & 1.073 & - \\
\hline 14 & Leitha $^{\text {a }}$ & 2.118 & Schwarza \\
\hline 15 & Rabnitz $^{\text {a }}$ & 777 & Einser-Kanal \\
\hline 16 & $\operatorname{Raab}^{\mathrm{a}}$ & 4.416 & Lafnitz, Pinka \\
\hline 17 & Mur ${ }^{a}$ & 9.908 & Taurach, Pöls, Liesing, Mürz, Kainach, Sulm \\
\hline 18 & Drau $^{\mathrm{a}}$ & 12.000 & Isel, Möll, Lieser, Gail, Gurk, Lavant \\
\hline
\end{tabular}

beim letzten österreichischen DonauPegel liegt. Der dominierende Fluss ist die Donau (ICPDR 2020; Prohaska et al. 2020), welche im äußersten Westen des Projektgebiets bei Donaueschingen entspringt (Abb. 1 , ca. $8,15^{\circ} \mathrm{E} / 48,10^{\circ} \mathrm{N}$ ). Die Einzugsgebiete der Hauptzubringer der Donau dienen der Unterteilung in 18 verschiedene Flussgebiete (Tab. 1). Eine Übersicht des von LamaH abgedeckten Bereichs, inkl. der Flussgebiete sowie der Abflusspegel mit der jeweiligen Pegelnullpunkthöhe, kann der Abb. 1 entnommen werden. Alle Flussgebiete außer Flussgebiet 1 (Rhein) und 11 (Moldau) sind Teil des Donaueinzugsgebiets. Gebiet 1 umfasst das Einzugsgebiet des Alpenrheins, also jenen Teil des Rheins von der Quelle bis zum Bodensee, während Gebiet 11 das österreichische Einzugsgebiet der Moldau (Elbe) abdeckt.

\section{Einzugsgebietsabgrenzungen}

Die Grundlage zur Erstellung der meteorologischen Zeitreihen sowie der Einzugsgebietseigenschaften waren meist globale Datensätze, aufbereitet entweder in Raster- oder Vektorform. LamaH ist so konzipiert, dass eine Einzugsgebietseigenschaft oder ein Zeitschritt einer meteorologischen Zeitreihe üblicherweise das Mittel des orographischen Einzugsgebiets repräsentiert. Daher war eine Aggregierung der räumlich verteilten Informationen der entsprechenden Ausgangsdatensätze erforderlich. Ausgangspunkt für die Erstellung der Aggregationsflächen waren Teileinzugsgebiete, die aus dem digitalen hydrologischen Atlas Österreich (HAO 2007) und darüber hinaus aus dem HydroATLAS (Linke et al. 2019; für Gebiete, die vom HAO nicht abgedeckt werden) entnommen wurden. Die Teileinzugsgebiete der beiden Datenquellen wurden miteinander vereinigt und bei Bedarf an die Pegelstandorte angepasst. Anschließend erfolgte getrennt für jeden Pegel die Vereinigung aller (kleineren) Teileinzugsgebiete, welche Teil des jeweiligen Einzugsgebiets sind. Jedem Pegel wird somit eine Aggregationsfläche zugeordnet, welche das jeweilige orographische Einzugsgebiet abbildet. Wir bezeichnen diese $\mathrm{Me}$ thode der Teileinzugsgebietsaggregation folglich und im Datensatz selbst als „Einzugsgebietsabgrenzung A“. Die Plausibilität der Einzugsgebietsabgrenzungen wurde an dieser Stelle überprüft, indem der Quotient aus der Aggregationsfläche und der offiziell,
z.B. in den Metadaten der Pegel, ausgewiesenen Einzugsgebietsfläche gebildet wurde (Attribut „area_ratio“" in Tab. 3 im Anhang; Standardabweichung 0,026 ; Spanne von 0,89 bis 1,34 ). Einzugsgebiete mit größerer Abweichung wurden manuell überprüft. Die mediane Einzugsgebietsgröße beträgt bei Anwendung von Einzugsgebietsabgrenzung A über alle 859 Einzugsgebiete $178 \mathrm{~km}^{2}$, mit einer Bandbreite von 3,9 bis $131.247 \mathrm{~km}^{2}$. Bei den bereits publizierten CAMELS-Datensätzen kam ausschließlich Einzugsgebietsabgrenzung A zur Anwendung. Der Vorteil dieser Einzugsgebietsabgrenzung ist die Unabhängigkeit der Einzugsgebiete untereinander, da das orographische Einzugsgebiet der jeweiligen Pegel widergespiegelt wird. Speziell bei Pegeln mit größeren Einzugsgebieten führt die Aggregierung bei Einzugsgebietsabgrenzung A aber zu einem nennenswerten Verlust an Information, da Variabilität sowie kleinräumige Charakteristika dadurch verloren gehen. Ein weiterer Nachteil ist die multiple, jedoch unterschiedlich gewichtete, Abbildung lokaler Merkmale in den Daten (siehe Überlappung in Abb. 2a). Daher wird Einzugsgebietsabgrenzung A durch eine Form der Abgrenzung (Einzugsgebietsabgrenzung B, auch 859 Einzugsgebiete) ergänzt, bei der die orographische Einzugsgebietsfläche des nächst flussauf liegenden Pegels (kann keiner, einer oder mehrere sein) von jener des aktuellen Pegels subtrahiert wird (Abb. 2b). Dadurch entstehen Zwischeneinzugsgebiete, welche Teil eines großen zusammenhängenden Flussnetzwerks werden. Die Abhängigkeit untereinander erfordert dabei eine Einzugsgebiets- bzw. Pegel-Hierarchie (Abb. 2b, Attribut „HIERARCHY“ in Tab. 3 im Anhang) sowie Information zur Upstream-Downstream-Beziehung („NEXTUPID“ bzw. „NEXTDOWNID“ in Tab. 3 im Anhang). Die mediane Einzugsgebietsgröße bei Anwendung von Einzugsgebietsabgrenzung $B$ beträgt $114 \mathrm{~km}^{2}$ mit einem Bereich von 1,3 bis $2.496 \mathrm{~km}^{2}$. Die dritte bei LamaH zur Verfügung gestellte Einzugsgebietsabgrenzung (im Text und im Datensatz weiters als Einzugsgebietsabgrenzung $\mathrm{C}$ bezeichnet) ist der Einzugsgebietsabgrenzung B recht ähnlich, jedoch werden hier nur Einzugsgebiete mit keiner oder geringer (anthropogener) Beeinflussung (454 Einzugsgebiete) einbezogen (Abb. 2c). Damit soll den Anwendern in nachfolgenden Studien eine zusam- 

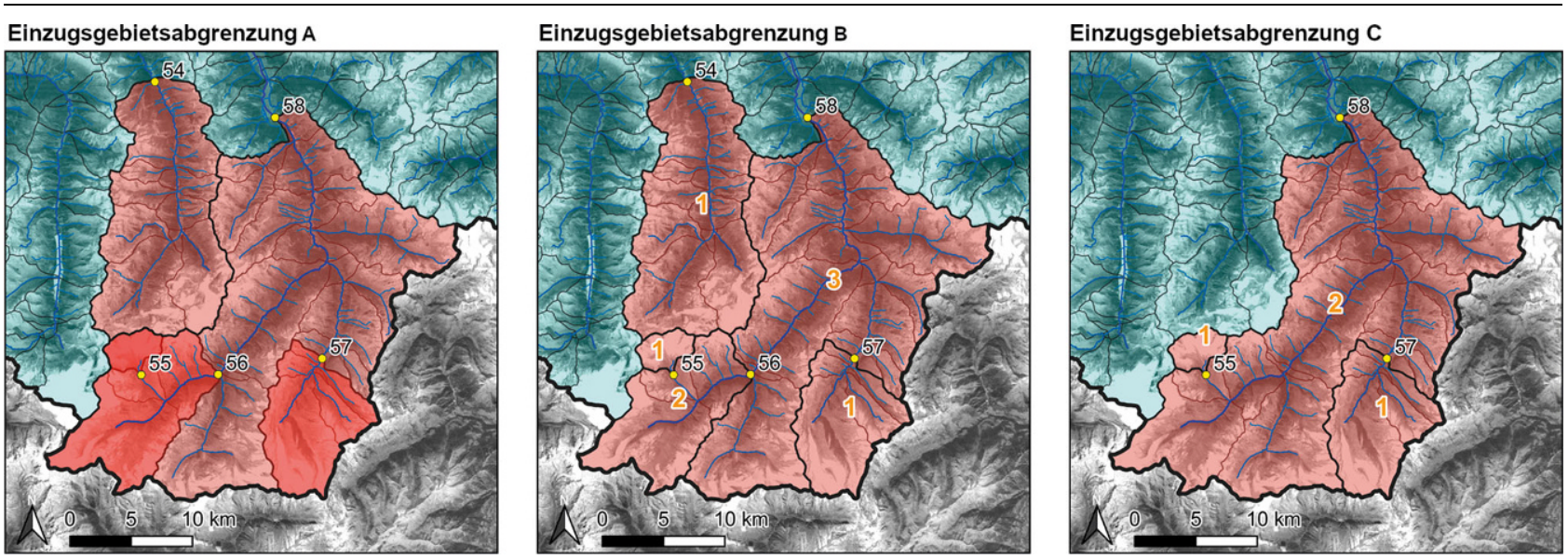

Abb. 2 Methoden der Einzugsgebietsabgrenzung an einem Beispiel erklärt: Die nicht-aggregierten Teileinzugsgebiete innerhalb des Projektgebiets sind mit einem blauen Farbton hinterlegt und von einer dünnen schwarzen Linie umrandet, während aggregierte Teileinzugsgebiete mit einem roten Farbton gekennzeichnet und einer mitteldicken schwarzen Linie umrandet sind. Die Grenze des Projektgebietes ist anhand der dicken schwarzen Linie dargestellt. Die Nummern mit schwarzer Schriftfarbe stellen die ID-Nummer des jeweiligen Pegels dar, während die orangen Nummern die Hierarchie kennzeichnen (Plot B und C). Einzugsgebietsabgrenzung $A$ (analog zu den bekannten CAMELS-Datensätzen): Die Aggregationsfläche entspricht dem orographischen Einzugsgebiet eines Pegels. Dabei ergeben sich Überlappungen mit den Aggregationsflächen der flussabwärts gelegenen Pegel. Im Plot A wird daher die Aggregationsfläche von Pegel 56 und 57 von jener des Pegels 58, sowie die Aggregationsfläche des Pegels 55 von jener der Pegel 56 und 58 überlappt (erkennbar an den unterschiedlichen roten Farbtönen). Einzugsgebietsabgrenzung B: Die Aggregationsflächen entsprechen bei dieser Methode der Differenzfläche (Zwischeneinzugsgebiete) zwischen dem orographischen Einzugsgebiet des jeweiligen Pegels sowie dem Einzugsgebiet der nächstflussaufwärts gelegenen Pegel. Konsequenterweise gibt es bei Einzugsgebietsabgrenzung B keine Überlappungen mehr, dafür wird aber eine Einzugsgebiets- und Pegelhierarchie notwendig. Im Plot B ist den Pegeln 54, 55 und 57 die Hierarchie 1 zugewiesen, da flussaufwärts kein Pegel vorhanden ist. Pegel 56 hat Hierarchie 2, da Pegel 55 flussaufwärts liegt. Hierarchie 3 erhält der Pegel 58, da mindestens ein flussaufwärts gelegener Pegel die Hierarchie 2 aufweist (Pegel56). Einzugsgebietsabgrenzung C:WieEinzugsgebietsabgrenzung B, wobeiabernurnichtoder gering beeinflusste Pegel (siehe Abschn. 5.8) berücksichtigt werden. Im Plot $C$ wird zur Veranschaulichung angenommen, dass Pegel 54 und 56 stark beeinflusst sind. Folglich werden diese beiden Pegel von der Einzugsgebietsabgrenzung ausgeschlossen und die Aggregationsfläche des Pegels 58 (nun Hierarchie 2) umfasst nun auch die Zwischeneinzugsgebietsfläche (Differenzfläche) des Pegels 56. Quelle des SatellitenHintergrundbildes: Google @ 2020 TerraMetrics, Kartendaten @ 2020, Quelle des Gewässernetzes: Land Tirol (2020)

menhängende Datenbasis von hydrologischen Mustern zur Verfügung gestellt werden, die den natürlichen Bedingungen nahekommen. Die (anthropogene) Beeinflussung der Einzugsgebiete bzw. Abflussmessung wird in Abschn. 5.8 näher erläutert.

\section{Hydrometeorologische Zeitreihen}

\subsection{Abflusszeitreihen}

LamaH enthält tägliche und stündliche Abflusszeitreihen für 882 verschiedene Pegelstandorte in 4 verschiedenen Ländern (Österreich, Deutschland, Schweiz und Tschechische Republik). Die Differenz zu den 859 Einzugsgebieten kann mit der Nichtberücksichtigung von 23 Pegeln, welche zum Großteil kein klar abgrenzbares Einzugsgebiet aufweisen (z. B. Pegel an künstlichen Kanälen oder unterhalb großer Karstquellen), bei der Einzugsgebietsabgrenzung erklärt werden (siehe Abschn. 5.8). Mit Daten für
609 Pegel stammt der Großteil vom Hydrographischen Zentralbüro Österreich (HZB 2020). Die gewässerkundlichen Landesdienste der deutschen Bundesländer Bayern (GKD 2020) und BadenWürttemberg (LUBW 2020) stellten 125 bzw. 61 Abflusszeitreihen zur Verfügung. 25 Abflusszeitreihen wurden vom hydrologischen Amt der Schweiz (BAFU 2020) bezogen, während Zeitreihen für 61 Pegel vom Tschechischen Hydrometeorologischen Institut (CHMI 2020) bereitgestellt wurden. Im Hinblick auf eine deutlich leichtere Verarbeitbarkeit wurden die Zeitreihenformate der verschiedenen Dienste vereinheitlicht. Attribute, welche die Abflusspegel sowie deren Zeitreihen spezifizieren, sind in Tab. $3 \mathrm{im}$ Anhang aufgelistet. Die Einheit der Abflusszeitreihen wurde mit $\mathrm{m}^{3} \mathrm{~s}^{-1}$ festgelegt, eine Umrechnung in die Abflusshöhe kann umgehend anhand der bereitgestellten Einzugsgebietsfläche durchgeführt werden.

Die kontinuierlichen Abflusszeitreihen sind überwiegend nur indirekt mittels sogenannter Wasserstands-Abfluss-Beziehungen vom gemessenen Wasserstand abgeleitet. So kann es beispielsweise nach Hochwasserereignissen mit starker Geschiebeführung aufgrund des geänderten Gerinneprofils $\mathrm{zu}$ einer ungenauen Ermittlung des Abflusses kommen. In der Regel wird jedoch versucht, diese Fehlerquelle durch eine regelmäßige Anpassung der Wasserstands-Abfluss-Beziehung $\mathrm{zu}$ minimieren. Zwar ist die Anpassungshäufigkeit dieser WasserstandsAbfluss-Beziehungen nicht öffentlich verfügbar, wo differenziert (Bayern und Tschechien) wurden aber nur Pegel von der höchsten Güteklasse in den Datensatz einbezogen. Oftmals stehen für Zeitreihen mit täglicher Auflösung längere Beobachtungszeiträume als für jene mit stündlicher Auflösung zur Verfügung. Daher können die Abflusszeitreihen meist getrennt nach zeitlicher Auflösung von den angeführten hydrographischen Ämtern bezogen werden. Wir haben jedoch in der Re- 


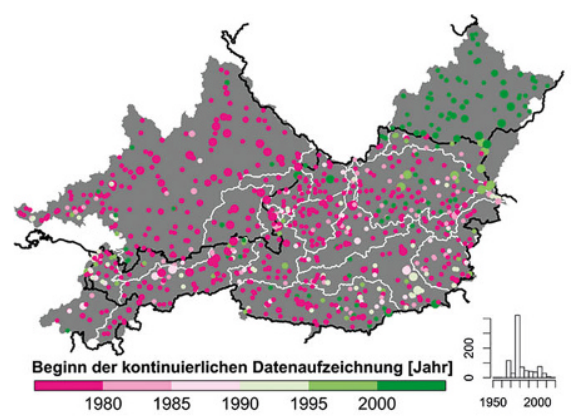

a

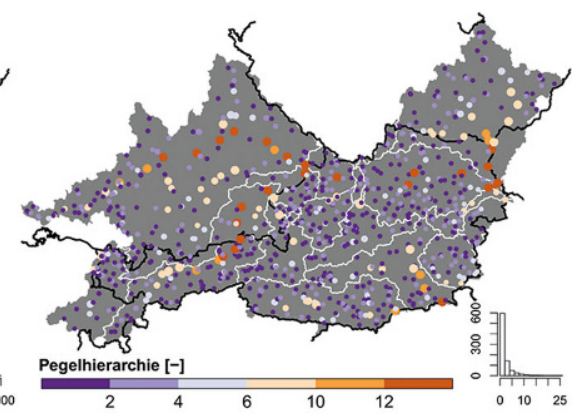

Abb. 3 Übersicht einer Auswahl der pegelbezogenen Attribute. Die Größe der Kreise ist dabei proportional zur Einzugsgebietsfläche. Das Histogramm zeigt die Verteilung der 859 Attributswerte an

gel nur die Zeitreihen mit stündlicher Auflösung angefragt und daraus die Tageszeitreihen abgeleitet. Anwendung fand dieser Ansatz bei Abflussreihen aus Österreich, Deutschland und der Schweiz, da dort auch die Zeitreihen mit stündlicher Auflösung meist recht lange Aufzeichnungszeiträume aufweisen. Abb. 3a zeigt, dass die meisten Pegel eine kontinuierliche (stündliche) Datenaufzeichnung seit den späten 1970er-Jahren haben (Histogramm in Abb. 3a). Im Gegensatz dazu wurden die Zeitreihen aus der Tschechischen Republik sowohl mit täglicher als auch mit stündlicher Auflösung angefragt, da die kontinuierliche Zeitreihe dort erst im Jahr 2005 beginnt. Die Abflusszeitreihen wurden im Datensatz auf den Zeitraum 1981 bis 2017 begrenzt, da seit 1981 die verwendeten meteorologischen ERA5Land-Forcings verfügbar sind und 2017 das letzte Jahr mit geprüften Abflussdaten des Hydrographischen Zentralbüros Österreichs zum Zeitpunkt der Anfrage war.

Obwohl der genaue Umfang der Datenüberprüfung durch die Mitarbeiter der verschiedenen hydrographischen und gewässerkundlichen Dienste nicht weiter spezifiziert ist, haben wir $\mathrm{zu}$ jedem Zeitschritt der Abflusszeitreihe ein Attribut hinzugefügt, welches den Prüfstatus beschreibt (Attribut „ckhs“ in Tab. 3 im Anhang). Die Abflussdaten aus Österreich, Tschechien sowie der Schweiz liegen im Zeitraum 1981 bis 2017 ausschließlich geprüft vor, während jene aus Bayern oft nur bis in die Jahre 2014, 2015 oder 2016 als geprüft gekennzeichnet sind. Abflussdaten aus Baden-Württemberg sind dagegen meist erst ab dem Jahr 2010 mit dem Status „geprüft“ versehen. Bei einigen Zeitreihen konnten nach Erhalt auch Lücken festgestellt werden. Um die Anzahl dieser Lücken zu reduzieren, wurden maximal 6 aufeinanderfolgende Lücken (also $6 \mathrm{~h}$ ) bei unserer Datenaufbereitung mittels linearer Interpolierung aufgefüllt. Darüber hinaus verbleibende Lücken wurden mit der Zahl -999 gekennzeichnet. Das Ausmaß der verbleibenden Lücken in den kontinuierlichen Abflusszeitreihen wird durch das Attribut „gaps_post“ in Tab. 3 im Anhang dokumentiert sowie in Abb. 3b dargestellt. Dabei zeigt sich, dass die Pegel mit sehr geringem Lückenanteil $(<0,1 \% 0)$ vor allem in Österreich, Tschechien und der Schweiz zu finden sind. Etwa $80 \%$ der 882 Pegel weisen nach der Verarbeitung keine Lücken in ihren kontinuierlichen Zeitreihen auf. Die Zeitschritte mit Lücken vor einer etwaigen Interpolation sind in separaten Dateien aufgelistet, die dem Datensatz der Vollständigkeit halber beigefügt sind. Die räumliche Verteilung der Pegelhierarchien, abgeleitet aus der Einzugsgebietsabgrenzung B, ist in Abb. 3c dargestellt. Ca. $50 \%$ aller Pegel weisen eine Hierarchie von 1 auf, haben also keinen anderen Pegel flussaufwärts. Die höchste Pegelhierarchie weist mit 26 der letzte Donaupegel auf österreichischem Territorium auf (ID 399).

\subsection{Meteorologische Zeitreihen}

Aufgrund des Umfangs des global abdeckenden ERA5-Land-Datensatzes (Muñoz Sabater et al. 2021) des ECMWF (European Centre for Medium-Range Weather Forecasts bzw. Europäisches Zentrum für mittelfristige Wettervorhersage) konnten lückenlose Zeitreihen für 15 hydrologisch relevante Variablen und 39 Jahre abgeleitet werden (Tab. 4 im Anhang). ERA5-Land ist ein Derivat der terrestrischen Komponenten der ERA5-Klimareanalyse (Hersbach et al. 2020), wobei jedoch einige
Weiterentwicklungen angewendet werden (Muñoz Sabater et al. 2021; Yang und Giusti 2020). In erster Linie sind dies ein Interpolationspaket für eine feinere zeiträumliche Auflösung, eine zusätzliche Seehöhenanpassung der meteorologischen Felder sowie effizientere Möglichkeiten für die Einspielung von Updates. ERA5-Land weist eine räumliche Auflösung von 0,1 Bogengrad (ca. $9 \times 11 \mathrm{~km}$ in den Breitengraden des Projektgebiets) im Vergleich zur Rastergröße von ERA5 mit 0,25 Bogengrad auf. Die zeitliche Auflösung von ERA5Land liegt bei $1 \mathrm{~h}$, während ERA5 nur eine 3-stündige Auflösung aufweist. ERA5-Land verwendet keine Datenassimilation (Anpassung an Beobachtungen), Beobachtungen sind aber indirekt über die assimilierten atmosphärischen Felder von ERA5 implementiert (Hennermann und Guillory 2020; Yang und Giusti 2020). Gemäß den Bestimmungen des ECMWF ist für ERA5-Land eine Unsicherheitsabschätzung vorgesehen (Muñoz Sabater 2019b; Muñoz Sabater et al. 2017), welche zum Zeitpunkt der Verfassung des Artikels (Januar 2021) aber noch nicht verfügbar war.

Die meteorologischen Zeitreihen wurden für alle 3 Formen der Einzugsgebietsabgrenzung (A/B/C) ermittelt. Dazu wurden die (Zwischen-)Einzugsgebiete der jeweiligen Einzugsgebietsabgrenzungen mit dem Raster von ERA5-Land verschnitten, um die Aggregation (arithm. Mittelung) der meteorologischen Variablen flächengewichtet durchführen zu können. Wie bereits in der Einleitung erwähnt, möchten wir auch auf mögliche Unsicherheiten der publizierten Daten hinweisen. Dazu wurden die Komponenten der Wasserbilanz für den Zeitraum 01.10.1989 bis 30.09.2009 ermittelt und in einer Grafik aufgetragen (Abb. 4a). Werte von Einzugsgebieten, die durch Wasserüber- 


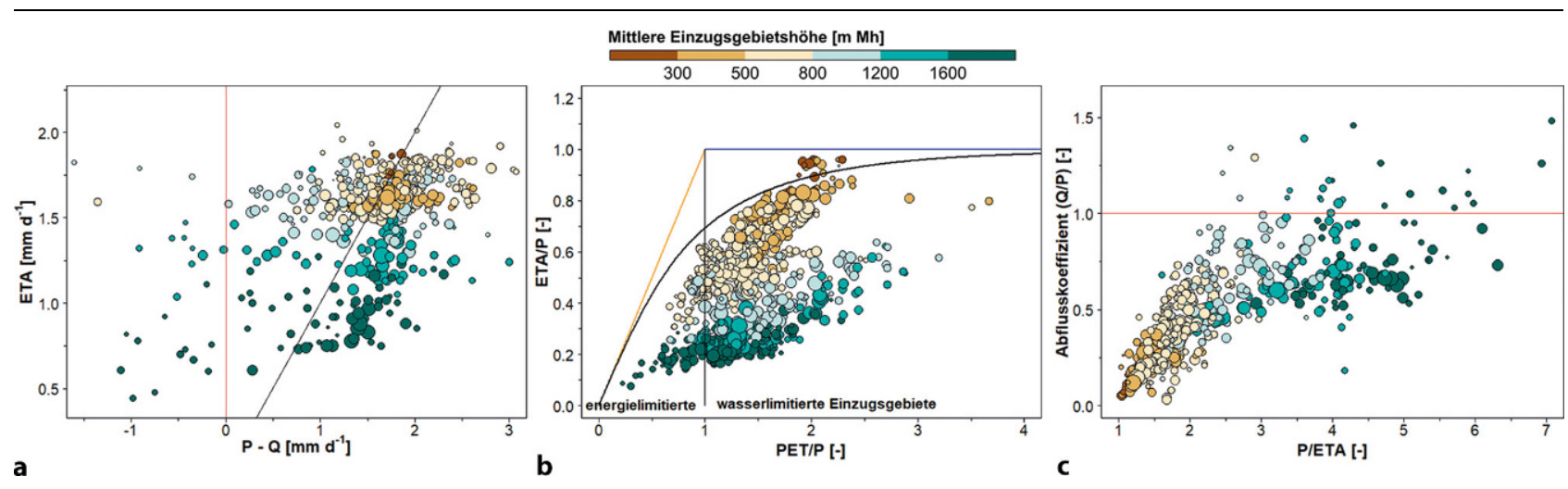

Abb. 4 Auswertungen zur Wasserbilanz für die hydrologischen Jahre 1990 bis 2009 sowie Einzugsgebietsabgrenzung A (Abb. $2 a)$. a Aktuelle Evapotranspiration (ETA) von ERA5-Land als eine Funktion der Differenz vom Niederschlag (P) von ERA5-Land und der gemessenen Abflusshöhe (Q). b Die Budyko-Kurve veranschaulicht, ob die Evapotranspiration eines Einzugsgebiets energielimitiert $(P E T / P<1)$ oder wasserlimitiert (PET/P > 1) ist. Plot c zeigt den Abflusskoeffizienten als eine Funktion des Quotienten vom Niederschlag und der aktuellen Evapotranspiration vonERA5-Land. InPlota, bund c werden Wertenurfürjene Pegel gezeigt, deren AbflussZeitreihe die Periode 01.10.1989 bis 30.09.2009 umfasst (717 Pegel). Weiters werden in den Plots a und c nur Werte für jene Pegel aufgenommen, welche nicht durch künstliche Wasserüberleitung oder -ableitung, größere Karstquellen oder starke Infiltration beeinflusst sind (594 Pegel, siehe Tab. 2). Die schräge schwarze Linie in a stellt die 1:1-Gerade dar, während die vertikale schwarze Linie in b die Grenze zwischen energie- und wasserlimitierten EZG bildet. Die roten Linien in a und c zeigen physikalische Limitierungen. Die schräge orange Linie in b indiziert das Energielimit, während die blaue horizontale Linie das Wasserlimit und die schwarze kurvige Linie die Budyko-Kurve darstellt. Die Größe des Kreises ist proportional zum Einzugsgebiet, dessen Farbgebung ist ein Indikator für die mittlere Seehöhe des Einzugsgebiets (siehe Legende)

leitung, -ableitung, große Karstquellen oder hohe Infiltration beeinflusst sind (siehe Abschn. 5.8), werden zwecks einer objektiveren Interpretation in Abb. 4a,c nicht dargestellt. Bei langfristigen Wasserbilanzen kann die künstliche Speicherung im Einzugsgebiet meist vernachlässigt werden. Die Differenz zwischen langjährigem mittlerem Niederschlag (P) und Abflusshöhe (Q) sollte bei den verbleibenden Einzugsgebieten gleich der aktuellen Evapotranspiration (ETA) sein, also auf der 1:1Linie in Abb. 4a liegen. Gründe für die doch recht starke Streuung um dieses Ideal (Pearson-Korrelation $\mathrm{R}=0,30$ ) liegen mitunter in einer unzureichenden Erfassung des Niederschlags oder der aktuellen Evapotranspiration durch ERA5-Land, einer ungenauen Erfassung des Abflusses (z.B. starker, nicht erfasster Grundwasserbegleitstrom oder Änderung des Flussprofils am Pegel und damit inadäquate WasserstandsAbfluss-Beziehung), einer deutlichen Abweichung des orographischen vom hydrographischen Einzugsgebiet (unterirdische Zuflüsse und Abflüsse, speziell in Karstgebieten) oder bei vorhandenen Gletschern in einer negativen Massenbilanz ebendieser (Lambrecht and Kuhn 2007; Kuhn 2004; Kobolschnig und Schöner 2011; Oerlemans et al. 1998; WGMS 2005). Auch die Verwendung anderer Niederschlagsdatensätze wie CHIRPS Daily v2 (Funk et al. 2015) $(\mathrm{R}$ zwischen $(\mathrm{P}-\mathrm{Q})$ und $\mathrm{ETA}=0,34)$ oder MSWEP v2.2 (Beck et al. 2017, 2019) $(\mathrm{R}=0,26)$ führt $\mathrm{zu}$ keiner wesentlich besser erfüllten langjährigen Wasserbilanz. Die mittleren Summen der aktuellen Evapotranspiration von ERA5Land sowie die Abhängigkeit von der Seehöhe scheinen im Vergleich zu anderen Studien jedoch recht plausibel zu sein (Abb. 4a; HAO 2007, Karte 3.3; Herrnegger et al. 2012, Abb. 20). Negative Differenzen von mittlerer Niederschlags- und Abflusshöhe (Abb. 4a) und damit Abflusskoeffizienten >1,0 (Abb. 4c, 32 von 594 Einzugsgebiete) sind hauptsächlich in höhergelegenen Regionen (Abb. 8d, evtl. negative Massenbilanz Gletscher) sowie Einzugsgebieten mit hohem Anteil an karbonatischem Sedimentgestein (Abb. 11c, Indikator für Karst) vorhanden. Da ERA5-Land indirekt über die assimilierten atmosphärischen Felder von ERA5 In-situ-Beobachtungsdaten einbezieht (Yang und Giusti 2020), könnte auch ein systematischer Messfehler einer verwendeten terrestrischen Station die Erklärung für einen $\mathrm{zu}$ geringen mittleren Niederschlag im betreffenden Gebiet sein (Herrnegger et al. 2018). Die einzelnen Komponenten der Wasserbilanz sind jedenfalls dem Datensatz einzugsgebietsweise beigefügt, da diese Auswertung bei der Erklärung etwai- ger Abweichungen bei einer späteren Modellierung sehr hilfreich sein kann.

Die Budyko-Kurve (Abb. 4b; Budyko 1974) beschreibt die Beziehung vom Verhältnis aktuelle Evapotranspiration (ETA)/Niederschlag (P) zum Verhältnis potenzielle Evapotranspiration (PET)/Niederschlag und indiziert, ob die Verdunstung eines Einzugsgebiets durch Energie oder Wasser limitiert ist. Idealerweise sollten alle Punkte in der Nähe der Budyko-Kurve liegen. Die Abweichung von diesem Idealfall ist in erster Linie durch eine deutlich $\mathrm{zu}$ hohe PET von ERA5-Land über den nahezu kompletten Höhenbereich zu erklären. So weisen beispielsweise $98 \%$ aller 859 Einzugsgebiete mittlere PETJahressummen von über $1000 \mathrm{~mm}$ auf. Da diese mittleren PET Jahressummen in den Breiten des Projektgebiets nicht realistisch sind (HAO 2007, Karte 3.2; Herrnegger et al. 2012, Abb. 17), wurde die potenzielle Evapotranspiration von ERA5-Land bei LamaH nicht inkludiert. Der Abflusskoeffizient (Q/P) als eine Funktion des Verhältnisses vom mittleren Niederschlag zur aktuellen Evapotranspiration (P/ETA) ist in Abb. 4c abgebildet, wobei die Seehöhenabhängigkeit klar ersichtlich ist. Abgesehen davon zeigen Einzugsgebiete mit geringerer mittlerer Seehöhe weniger Streuung als solche mit höherer. 

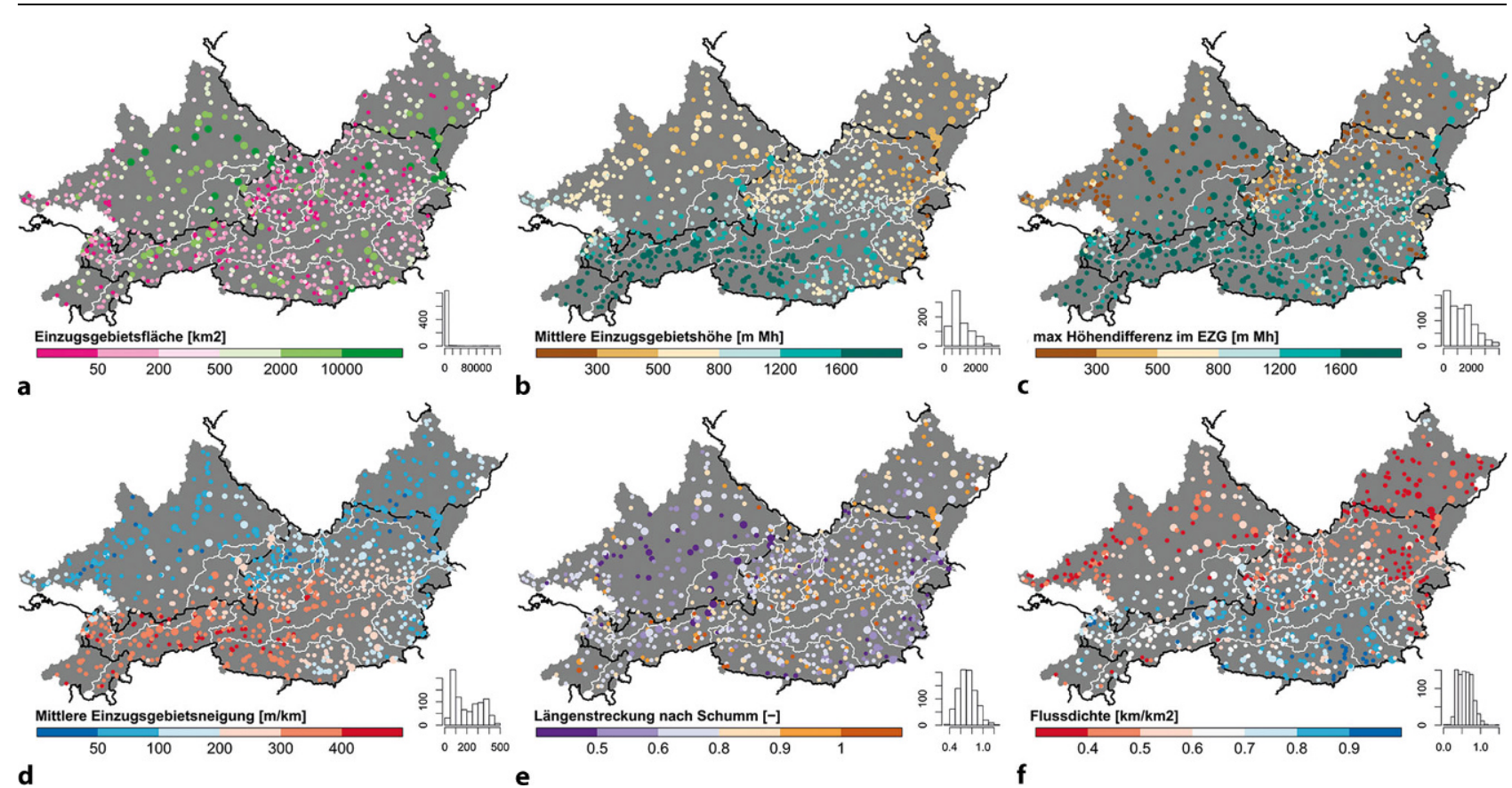

Abb. 5 Übersicht einer Auswahl der topographischen Attribute, welche die Eigenschaften des gesamten orographischen Einzugsgebiets darstellen (Einzugsgebietsabgrenzung A, Abb. 2a). Die Größe der Kreise ist dabei proportional zur Einzugsgebietsfläche. Das Histogramm zeigt die Verteilung der 859 Attributswerte an

\section{Einzugsgebietseigenschaften}

Die verschiedenen physio-geographischen Charakteristika eines Einzugsgebiets sowie dessen Wechselwirkungen spielen in Bezug auf Wasserspeicherung und -transport auf und unter der Erdoberfläche eine essenzielle Rolle (Blöschl et al. 2013). Das Spektrum der beeinflussenden Einzugsgebietseigenschaften reicht dabei unter anderem von den Bereichen Topographie, Klima, Hydrologie, Landbedeckung, Vegetation, Boden sowie Geologie bis hin zur Art und zum Grad der (anthropogenen) Beeinflussung des Abflussgeschehens in den Einzugsgebieten oder in der Pegelmessung. Einzugsgebietsattribute sind des Weiteren ebenfalls entscheidend, um Zusammenhänge zwischen verschiedenen Einzugsgebieten entlang mehrerer Gradienten zu bestimmen (Addor et al. 2017a; Falkenmark und Chapman 1989; Fan et al. 2019).

Für die Ableitung der verschiedenen Attribute werden meist frei verfügbare Datenätze mit globaler oder zumindest europäischer Abdeckung verwendet. Die Aggregierung der zeiträumlich verteilten Informationen dieser Ausgangsdatensätze erfolgt dabei jeweils für die 3 verschiedenen Einzugsgebietsabgrenzungen (A/B/C) nach Abschn. 3 und standardmäßig durch Berechnung des flächengewichteten arithmetischen Mittels (andernfalls indiziert). Die Datengrundlage von LamaH, Methoden der Aufbereitung, etwaige Unsicherheiten sowie die räumliche Verteilung der Einzugsgebietseigenschaften (Addor 2017b) werden in den nachfolgenden Unterkapiteln näher erläutert. Die einzelnen Attribute sind im Anhang unter Angabe einer genaueren Beschreibung, der Einheit sowie der Datenquellen tabellarisch angeführt.

\subsection{Topographische Attribute}

Wir haben diesbezüglich 10 Attribute berechnet, welche der Tab. 5 im Anhang entnommen werden können. Das Attribut „area_calc“ beschreibt die resultierende Aggregationsfläche, je nach angewendeter Form der Einzugsgebietsabgrenzung (siehe Abschn. 3). Bei Einzugsgebietsabgrenzung A sind ca. $34 \%$ aller 859 Einzugsgebiete (Aggregationsflächen) kleiner als $100 \mathrm{~km}^{2}$, $50 \%$ zwischen 100 und $1.000 \mathrm{~km}^{2}, 14 \%$ zwischen 1.000 und $10.000 \mathrm{~km}^{2}$ sowie rund $2,8 \%$ über $10.000 \mathrm{~km}^{2}$ groß. Große Einzugsgebiete weisen vor allem die Pegel an der Donau sowie jene an größeren Zubringern auf (Abb. 5a). Die Intention hinter der Anwendung mehrerer Einzugsgebietsabgrenzungen war unter anderem eine Verringerung der Aggregationsflächen und damit eine repräsentativere Abbildung lokaler Gegebenheiten und Erhaltung der natürlichen Variabilität. Bei Einzugsgebietsabgrenzung B weisen ca. $45 \%$ aller 859 Aggregationsflächen eine Fläche von $100 \mathrm{~km}^{2}, 52 \%$ zwischen 100 und $1.000 \mathrm{~km}^{2}$ und nur 2,3\% eine Fläche über $1.000 \mathrm{~km}^{2}$ auf.

Ein wesentlicher Faktor für hydrologische Prozesse ist die Seehöhe, da diese auch viele andere Einzugsgebietseigenschaften unter anderem in den Bereichen Klima, Landbedeckung, Vegetation oder Bodenentwicklung beeinflusst (Addor et al. 2017a). So wird neben der mittleren Seehöhe eines Einzugsgebiets (Abb. 5b, „elev_mean“ in Tab. 5 im Anhang) auch der Median („elev_med“), die Standardabweichung („elev_std“), die Spannweite (maximale - minimale Seehöhe im Einzugsgebiet, Abb. 5c, „elev_ran“) sowie die mittlere Neigung (Abb. 5d, „slope_mean“) vom SRTM-Datensatz der NASA (Farr et al. 2007) abgeleitet. SRTM weist eine Rastergröße von $30 \mathrm{~m}$ auf und bietet weltweit einen maximalen absoluten vertikalen Fehler von $16 \mathrm{~m}$ bei einem Konfidenzintervall von $90 \%$, wobei die Genauigkeit mit zunehmender Seehöhe und Neigung abnimmt (Farr et al. 2007). 
Die Neigung wurde von der Geländehöhe vom SRTM-Datensatz mit dem Algorithmus nach Horn (1981) abgeleitet. Große mittlere Einzugsgebietshöhen sowie -neigungen sind vor allem in den Ostalpen zu erkennen, welche sich vom Südwesten bis zum mittleren Osten des Projektgebietes erstrecken. Dieses Gebiet ist hauptsächlich vom flacheren Alpenvorland sowie Regionen mit älteren geologischen Zonen umgeben.

Des Weiteren haben auch die Form des Einzugsgebiets sowie des Gewässernetzes Einfluss auf das Abflussgeschehen. In Hochwassersituationen spielt speziell bei größeren Einzugsgebieten die Zugsrichtung des Niederschlags in Relation zur Längsachse des Einzugsgebiets eine wesentliche Rolle. Daher wird neben der Distanz der Längsachse („mvert_dist“) auch der Winkel zwischen Nordrichtung und der Längsachse („mvert_ang“) als Attribut spezifiziert. In Kombination mit den beiden Windkomponenten von ERA5Land („10m_wind_u“, „10m_wind_v“" Tab. 4 im Anhang) lässt sich damit beispielsweise, zumindest grob, eine Aussage über diese Relation treffen. Darüber hinaus ist die Form des Einzugsgebiets für den Anstieg der Hochwasserwelle relevant. Das Attribut der Längenstreckung nach Schumm (1956) (Abb. 5e, „elon_ratio“) ist ein Indikator in Bezug auf die „Rundheit“ (je runder, desto höher sind die Werte) des Einzugsgebiets. Unregelmäßigkeiten wie z. B. große Einkerbungen in der Form des Einzugsgebiets können die Aussagekraft dieses Attributs allerdings reduzieren. Die Fließgewässerdichte (Abb. 5f, „strm_dens“) ist eine Funktion mehrerer Eigenschaften (z. B. Klima, Relief, Bodeneigenschaften, Geologie, Vegetation, Landnutzung sowie Vergletscherung oder Verkarstung) und kann daher ein recht aussagekräftiger Indikator für den Vergleich von Einzugsgebieten sein (Olden und Poff 2003). Die EU-Hydro-River Network Database (EEA 2019) wurde für die Berechnung der Fließgewässerdichte herangezogen, da diese feinaufgelöst und über das Projektgebiet von LamaH konsistent ist.

\subsection{Klimatologische Attribute}

LamaH beinhaltet 12 verschiedene Attribute, welche Aspekte der klimatischen Eigenschaften widerspiegeln (Tab. 6 im Anhang). Diese Attribute wurden überwiegend aus den meteorologischen Zeitreihen von ERA5Land für den Zeitraum 01.10.1989 bis 30.09.2009 berechnet (Addor 2017b). Da die potenzielle Evapotranspiration von ERA5-Land deutlich zu hoch erscheint (Abschn. 4.2), wird als Alternative die Referenz-Evapotranspiration (ET0) von der Global Aridity Index and Potenzial Evapotranspiration (ET0) Climate Database v2 (Trabucco und Zomer 2019) verwendet, welche für den Zeitraum 1970 bis 2000 berechnet wurde. Die Referenz-Evapotranspiration ET0 beschreibt die Verdunstungsfähigkeit der Atmosphäre für definierte Vegetationseigenschaften. Die potenzielle Evapotranspiration (PET) kann aus ET0 unter Verwendung von Korrekturfaktoren für Vegetations- und Bodeneigenschaften abgeleitet werden (Allen et al. 1998; Hargreaves 1994).

Langzeitliche klimatische Charakteristika werden durch den mittleren Niederschlag (Abb. 6a, „p_mean“ in Tab. 6 im Anhang), die mittlere Referenz-Evapotranspiration (Abb. 6b, „et0_mean“), die mittlere aktuelle Evapotranspiration („eta mean“) sowie den Ariditätsindex (Abb. 6c, „arid_1“) beschrieben, der den Quotienten aus mittlerer ET0 und Niederschlag darstellt. Abb. 6a zeigt die Niederschlagsstaulagen entlang des Alpennordrands sowie die Seehöhenabhängigkeit des mittleren Niederschlags recht gut. Prinzipiell ist der Westen des Projektgebiets aufgrund der stärkeren Beeinflussung durch ozeanisches Klima von höheren mittleren Niederschlagssummen geprägt. Die mittlere tägliche Referenz-Evapotranspiration (Abb. 6b, Pearson-Korrelation $R=-0,79$ ), die Aridität (Abb. 6c; $R=-0,67$ ) sowie auch der Anteil des Niederschlags, der als Schnee fällt (Abb. 6g; $R=0,96$ ) weisen ähnliche räumliche Muster wie jenes der mittleren Seehöhe des Einzugsgebiets (Abb. 6b) auf. Rund 14\% aller Einzugsgebiete, welche ausschließlich im Osten des Projektgebiets liegen, weisen eine Aridität (ET0/P) größer 1 auf.

Attribute, welche die Saisonalität beschreiben, sind der Anteil des Niederschlags, der als Schnee fällt (Abb. $6 \mathrm{~g}$, „frac_snow“), sowie der SaisonalitätsIndex, welcher auf Sinuskurven beruht, um den Niederschlagszyklus über das Jahr $\mathrm{zu}$ beschreiben (Abb. 6d, „p_season“). Ein höherer positiver Saisonalitäts-Index indiziert im Mittel höhere Niederschlagssummen während der Sommermonate, während Werte um 0 eine ausgeglichene Niederschlagsverteilung über das Jahr andeuten.

Obwohl langzeitliche und saisonale Indizes die allgemeine Klimatologie gut beschreiben, geben sie weniger oder keinen Einblick in relativ kurzfristige Ereignisse wie Trockenheit oder Starkregen. Daher wurden auch Attribute berechnet, welche die Häufigkeit von Starkniederschlägen (Tage pro Jahr mit min. 5-fachem mittlerem täglichem Niederschlag; Abb. 6e, „hi_prec_fr“) und Trockentagen (Tage pro Jahr mit max. $1 \mathrm{~mm} \mathrm{~d}^{-1}$ Niederschlag; Abb. $6 \mathrm{~h}$, „lo_prec_fr"), dessen mittlere Dauer (Abb. 6f, „hi_prec_du“/Abb. 6i, „lo_prec_du“), sowie die wahrscheinlichste Saison des Auftretens beschreiben („hi_prec_ti“/,lo_prec_ti“). Grund für die vermehrte Häufigkeit von Starkniederschlagstagen im Südosten des Projektgebiets (Abb. 6e) ist vor allem die Kombination aus relativ niederschlagsreichen konvektiven Regenereignissen während der Sommermonate und verhältnismäßig geringen Niederschlagssummen während des restlichen Jahres (Abb. 6d). Sowohl bei der mittleren Häufigkeit von Trockentagen (Abb. 6h; $\mathrm{R}=-0,62$ ) als auch bei der Dauer ebendieser (Abb. 6i; $R=-0,57$ ) kann ein räumlicher Zusammenhang mit der mittleren Höhe des Einzugsgebiets (Abb. 5b) beobachtet werden. Die wahrscheinlichste Saison für Starkniederschläge ist für $89 \%$ aller 859 Einzugsgebiete der Sommer (Juni, Juli und August), während der Winter (Dezember, Januar und Februar) für $89 \%$ der Einzugsgebiete die häufigste Jahreszeit für Trockentage ist.

\subsection{Hydrologische Attribute}

Die Abflusszeitreihen der Pegel werden anhand 14 verschiedener Attribute charakterisiert (Tab. 7 im Anhang), welche für die Periode 01.10.1989 bis 30.09.2009 berechnet worden sind (Addor 2017b). Dabei wurden die Indizes für jene Pegel berechnet, welche den angeführten Untersuchungszeitraum abdecken (717 Pegel). Die Auswertungen für den gesamten Aufzeichnungszeitraum der einzelnen Pegel (erster 01.10. der jeweiligen Zeitreihe ab 1981 bis 30.09.2017) sind dem Datensatz zusätzlich beigefügt. Die hydrologischen Attribute können in solche eingeteilt werden, welche langzeitliche Charakteristika, Saisonalität sowie eher kurzfristige Situationen wie Hoch- und Niedrigwasser beschreiben. 

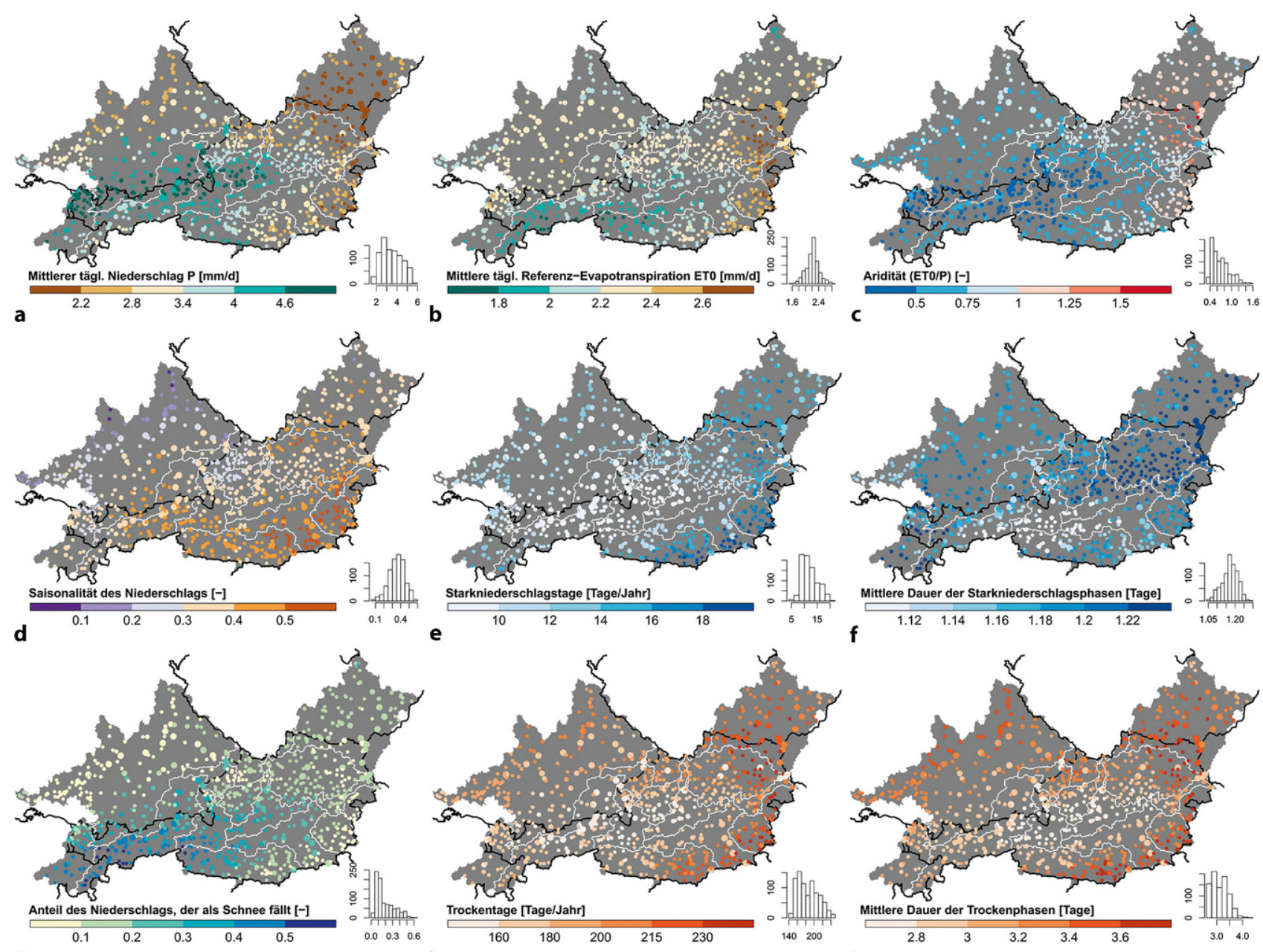
g

h

i

Abb. 6 Übersicht der klimatologischen Attribute, welche die Eigenschaften des gesamten orographischen Einzugsgebiets darstellen (Einzugsgebietsabgrenzung A, Abb. 2a). Die Größe der Kreise ist dabei proportional zur Einzugsgebietsfläche. Das Histogramm zeigt die Verteilung der 859 Attributswerte an

Die Aridität allein kann schon ein guter Hinweis für das Abflussaufkommen in einem Einzugsgebiet sein (Arora 2002; Blöschl et al. 2013; Budyko 1974). Dies zeigt sich auch durch die ähnliche räumliche Verteilung der mittleren Abflusshöhe (Abb. 7a, „q_mean“ in Tab. 7 im Anhang, $\mathrm{R}=-0,70)$, sowie des Abflusskoeffizienten (Abb. 7b, „runoff_ratio“, $\mathrm{R}=-0,65)$ im Vergleich $\mathrm{zu}$ jener der Aridität (Abb. 7c). Der Abflusskoeffizient (Q/P) ist derjenige Anteil des Niederschlags, der langfristig nach Abzug von Evapotranspiration, Grundwasserfluss oder Speicheränderung an der Oberfläche abfließt. Erklärungen für Abflusskoeffizienten $>1$ können dem Abschn. 4.2 entnommen werden. Das Verhältnis von Basisabfluss zu Abfluss kann ein wertvoller Indikator zur Einzugsgebietsklassifizierung sein (Sawicz et al. 2011; Fan 2015), und wird weiterfolgend als Basisabfluss-Index („,baseflow_index“) bezeichnet. $\mathrm{Zu}$ beachten ist jedoch, dass dieser Index stark von der angewendeten Methode der Separation des Hydrographen abhängig ist (Beck et al. 2013; Chapman 1999; Eckhardt 2008). Daher kamen mit dem Ladson-Filter (Ladson et al. 2013, Abb. 7e), sowie dem Ansatz nach Tallaksen und Van Lanen (2004) zwei verschiedene Typen zur Separation des Hydrographen zur Anwendung. Die Abfluss-Niederschlags-Elastizität („stream_elas“) beschreibt die Trägheit der Änderung des mittleren Abflusses bei einer Änderung des mittleren Niederschlags (Sankarasubramanian et al. 2001). So würde beispielsweise ein Wert von 3 eine Änderung des mittleren Abflusses von $3 \%$ bei einer Änderung des mittleren Niederschlags von $1 \%$ andeuten. Eine relativ hohe Abfluss-
Niederschlags-Elastizität ist vor allem im Osten des Projektgebiets vorhanden (Abb. 7f). Der Anteil der Tage ohne Abfluss (nicht gezeigt, „zero_q_freq“) kann ein Hinweis auf starke Infiltration (z.B. Donauversickerung; Hötzl 1996), künstliche Wasserentnahme oder abgeklungenem Basisabfluss sein.

Die Saisonalität des Abflussgeschehens ist unter anderem durch das Attribut „hfd_mean“ beschrieben, welches die Anzahl der Tage seit Beginn des hydrologischen Jahres (01. Oktober) bis zur halben mittleren Jahresabflussfracht angibt (Court 1962). Eine höhere Anzahl an Tagen in Abb. 7c kann primär durch die Niederschlagsspeicherung durch Schnee (Abb. 6g) und Gletscher (Abb. 8d) erklärt werden. Die Variabilität des Abflusses (Abb. 7d, „slope_fdc“) wird innerhalb LamaH durch die Steigung der Dauerlinie zwischen log- 

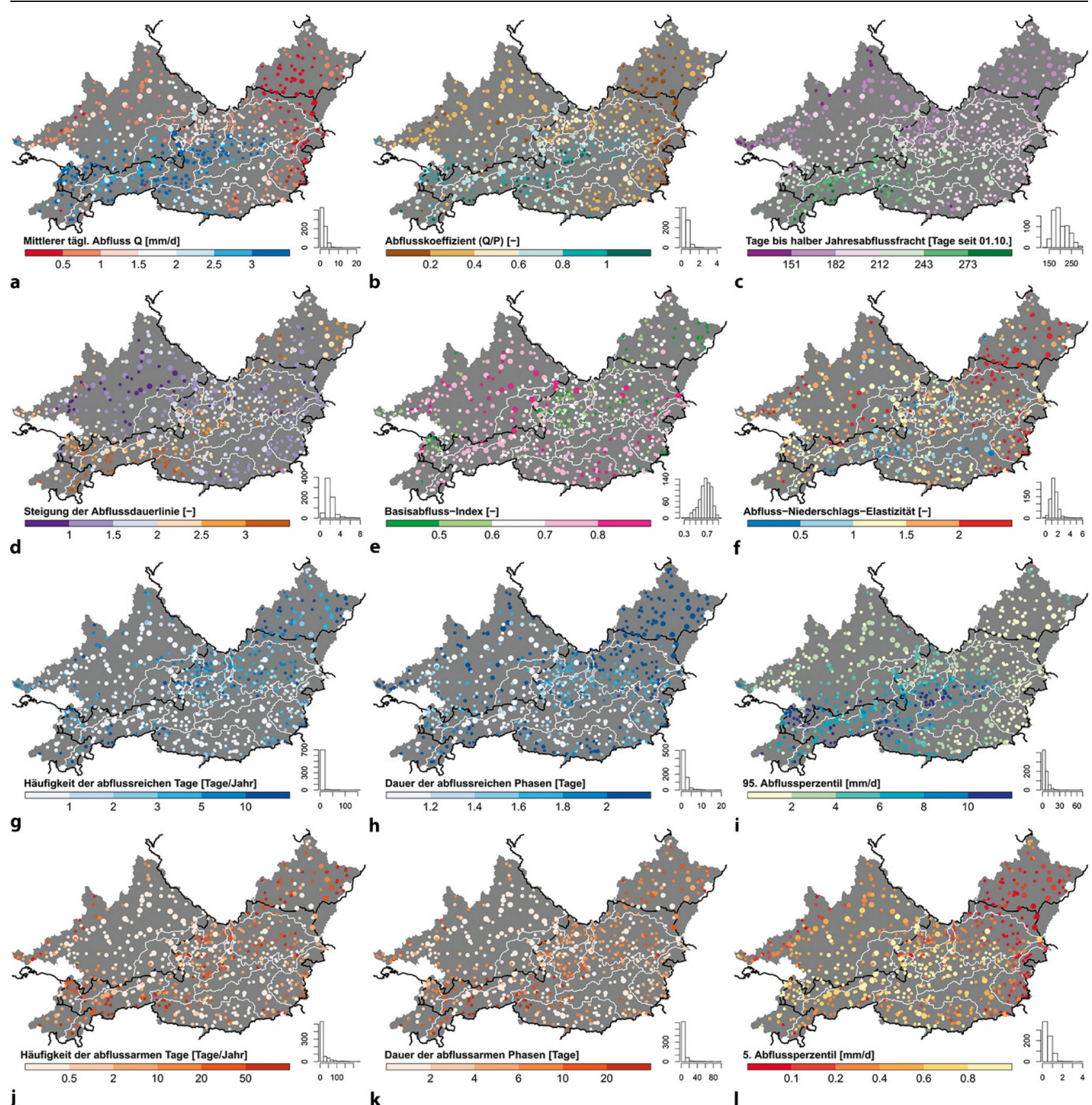
j

Abb. 7 Übersicht der hydrologischen Attribute. Es werden dabei nur jene Pegel in die Grafik aufgenommen, deren Zeitreihe die Periode 01.10.1989 bis30.09.2009 umfasst. Das Histogrammzeigt die Verteilung der 717 Attributswertean. Die Größe derKreise ist dabei proportional zur Einzugsgebietsfläche

transformierten 33. und 66. Abflussperzentil ausgedrückt (Sawicz et al. 2011). Hohe Werte sind dabei ein Indiz für eine hohe Abflussvariabilität über das Jahr, welche z.B. auf eine saisonale Wasserspeicherung in Form von Schnee (Abb. 6g) oder eine starke Reaktion des Abflusses auf Niederschlag zurückzuführen ist (Yokoo und Sivapalan 2011).

Außerordentliche Abflussereignisse wie Hoch- oder Niedrigwasser werden durch Indizes beschrieben, die die mittlere Häufigkeit (Abb. 7g, „high_q_freq“/ Abb. 7j, „low_q_freq“), Dauer (Abb. 7h, „high_q_dur"/Abb. 7k, „low_q_dur") und Magnitude abbilden. Der Schwellenwert für Hochwasser (>9-facher medianer Tagesabfluss) wird dabei nach Clausen und Biggs (2000) gewählt, jener für Niedrigwasser $(<0,2$-facher medianer Tagesabfluss) nach Olden und Poff (2003). Die Extremwasser-Magni- tuden werden durch das 95. (Hochwasser) sowie 5. (Niedrigwasser) Abflussperzentil ausgedrückt. Sowohl das Hochwasser-Q95 (Abb. 7i) als auch das Niedrigwasserperzentil $Q_{5}$ (Abb. 7l) weisen eine ähnliche räumliche Verteilung wie jene der Aridität (Abb. 6c; $\mathrm{R}=-0,62$ bzw. -0,63) auf. Die hydrologischen Indizes (Abb. 7) sind dabei räumlich weniger glatt als die klimatischen Indizes (Abb. 6) verteilt. Begründet werden 


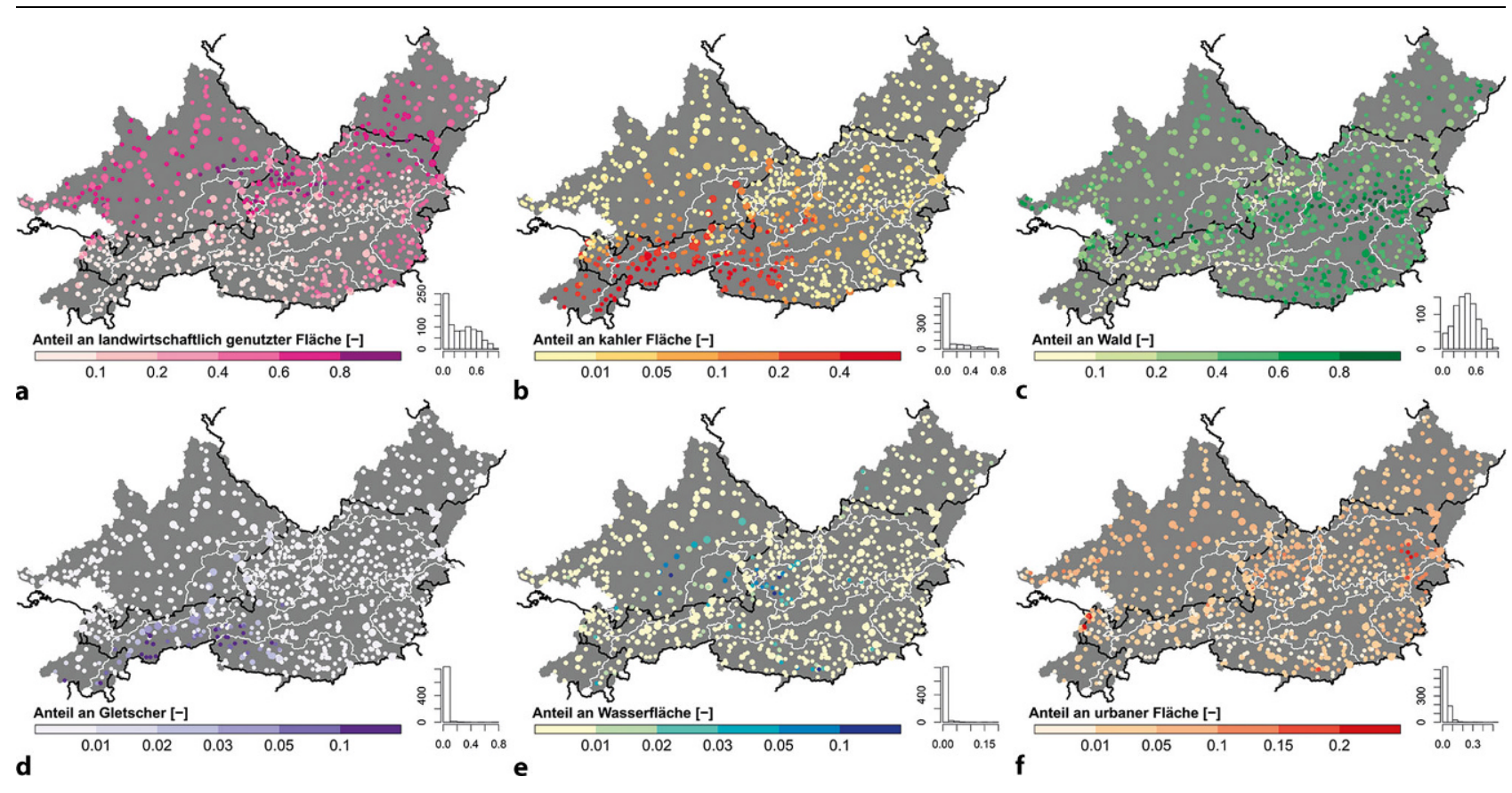

Abb. 8 Übersicht der Attribute zur Landnutzung, welche die Eigenschaften des gesamten orographischen Einzugsgebiets darstellen (Einzugsgebietsabgrenzung A, Abb. 2a). Die Größe der Kreise ist dabei proportional zur Einzugsgebietsfläche. Das Histogramm zeigt die Verteilung der 859 Attributswerte an

kann dies unter anderem mit der Beeinflussung der mitunter hochgradig nichtlinearen Abflussprozesse durch lokal heterogene Einzugsgebietseigenschaften oder mit Unsicherheiten bei der Abflussmessung (Addor et al. 2017a; Westerberg et al. 2016).

\subsection{Attribute zur Landbedeckung}

Alle Attribute, welche Bezug auf die Landbedeckung nehmen (Tab. 8 im Anhang), basieren auf dem Fernerkundungsdatensatz CORINE Land Cover 2012 mit einer räumlichen Auflösung von $100 \mathrm{~m}$ (CORINE 2012). CORINE ist eine Initiative der Europäischen Umweltagentur mit dem Ziel, die Landbedeckung des europäischen Territoriums sowie Änderungen davon mittels 6-jährigem Updatezyklus zu erfassen. Die grundlegenden technischen Spezifikationen, wie 44 Landklassen, 25 ha Mindestkartierungseinheit (MMU) für flächenhafte sowie $100 \mathrm{~m}$ Mindestbreite für lineare Strukturen, haben sich seit Beginn der Initiative nicht verändert und erleichtern somit Vergleiche über die Jahre (CORINE 2012). Anzumerken ist, dass kleinräumige Strukturen aufgrund der MMU von 25 ha nicht abgebildet werden. Weitere Einschränkungen könnten die Variabilität der Satellitenbildqualität und -inhalte, Schwie- rigkeiten bei der Einrichtung automatischer Konvertierungsprozesse und der Unterschied zwischen menschlicher Interpretationsfähigkeit sowie pixelbasierter Klassifizierung sein (Bossard et al. 2000). Die Zuverlässigkeit des Vorgängerdatensatzes CLC 2000 beträgt jedenfalls $87,0 \pm 0,8 \%$. Die geringste $\mathrm{Zu}$ verlässigkeit auf Klassenebene $(<70 \%)$ wurde dort für spärliche Vegetation (CLC-Klasse 333) angegeben (Büttner und Maucha 2006). Die vorherrschende Landnutzungsklasse „lc_dom“ wird durch die Mehrheit der einschließenden Raster-Zentroide bestimmt.

Wenig überraschend haben landwirtschaftlich genutzte Flächen (Abb. 8a "agr_fra“ in Tab. 8 im Anhang) vor allem in tiefer gelegenen Einzugsgebieten mit geringer mittlerer Neigung (Abb. 5d; $\mathrm{R}=-0,89$ ) hohe Flächenanteile. Eine diametrale Situation zeigt sich beim Anteil der kahlen Flächen (Abb. 8b, „bare_fra“), da die Vegetationsperiode in hohen Lagen sehr kurz ist und in diesen (alpinen) Gebieten eine hohe Geländeneigung gravitative Erosionsprozesse fördert. Bei der Berechnung des Waldanteils wurde, entsprechend den CAMELS-Datensätzen, nicht zwischen Laub- und Nadelwäldern differenziert. Der Waldanteil ist in der zentral-östlichen Region des Projektgebietes am höchsten (Abb. 8c, „forest_fra“), da dort
Landwirtschaft und Besiedlung weniger stark ausgeprägt sind und die Berge oft nicht höher als die Waldgrenze liegen. Einzugsgebiete mit einem relativ hohen Anteil an Gletschern (Abb. 8d, "glac_fra“) sind im Projektgebiet vor allem in den westlichen Ostalpen $\mathrm{zu}$ finden. Die Beeinflussung des hydrologischen Regimes durch Gletscher ist also vorranging in den Oberliegern der Flussgebiete Inn (Gebiet 3 in Abb. 1 sowie Tab. 1), Salzach (Gebiet 4) und Drau (Gebiet 18) bemerkbar. Hohe Anteile an Wasserfläche (Abb. 8e, „lake_fra“) können mit großen Seen, welche meist durch das Abklingen der letzten großen Eiszeit vor ca. 10.000 Jahren entstanden sind (v.a. im Alpenvorland), oder mit künstlichen Wasserreservoirs (v.a. in Tschechien) erklärt werden. Einzugsgebiete im Ballungsraum Wien (östlicher Teil des Flussgebiets 10) sowie im unteren Rheintal (nördlicher Teil des Flussgebiets 1) weisen recht hohe Anteile an urbaner Fläche auf (Abb. 8f, „urban_fra“). Die meisten Einzugsgebiete (ca. $74 \%$ ) haben jedoch einen Anteil an urbaner Fläche von weniger als $5 \%$.

\subsection{Attribute zur Vegetation}

Wir haben 6 verschiedene Einzugsgebietsattribute berechnet, die Vegetationsindizes in puncto Blattflächenindex 

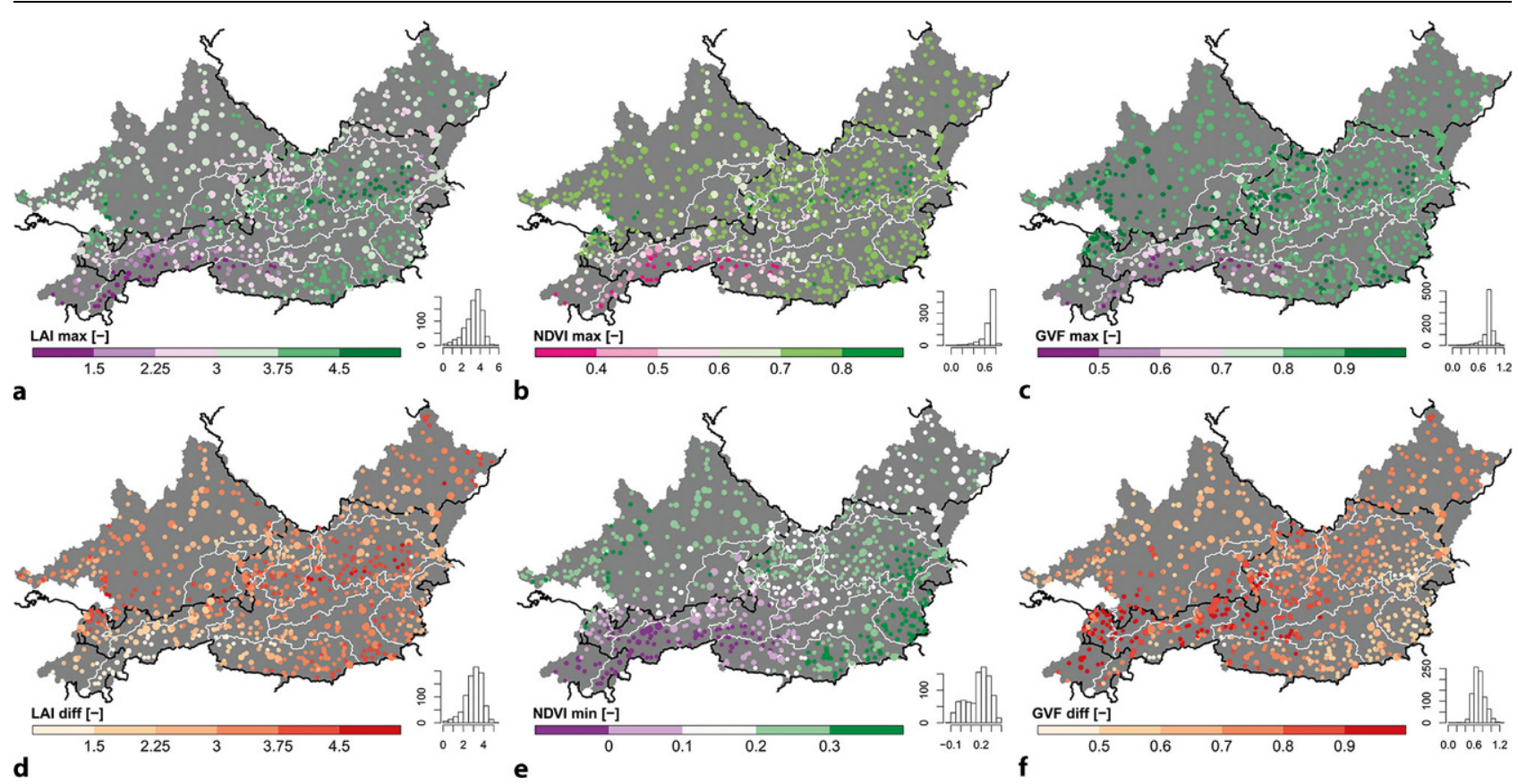

Abb. 9 Übersicht der Attribute zur Vegetation, welche die Eigenschaften des gesamten orographischen Einzugsgebiets darstellen (Einzugsgebietsabgrenzung A, Abb. 2a). Die Größe der Kreise ist dabei proportional zur Einzugsgebietsfläche. Das Histogramm zeigt die Verteilung der 859 Attributswerte an

(LAI - Leaf Area Index), Normalized Difference Vegetation Index (NDVI) und Green Vegetation Fraction (GVF) beschreiben (Tab. 9 im Anhang). Alle Vegetationsindizes basieren auf langfristigen Monatsmittelwerten, wobei entweder das Maximum, das Minimum oder die Differenz zwischen dem maximalen und dem minimalen Monatsmittelwert (basierend auf $12 \mathrm{Mo-}$ natsmittelwerten) verwendet wird. Die Prozessierung der zugrundeliegenden Fernerkundungsdatensätze erfolgte dabei mit der Plattform Google Earth Engine (GEEa 2021; GEEb 2021; Gorelick et al. 2017).

LAI repräsentiert die vertikale Vegetationsdichte und ist definiert als die Summe der einseitigen grünen Blattfläche pro Flächeneinheit bei Laubwäldern sowie die Hälfte der gesamten Nadelfläche pro Flächeneinheit bei Nadelwäldern. Die entsprechenden Attribute wurden vom Datensatz „MODIS MCD15A3H“ abgeleitet, welcher eine 4-Tages-Komposition mit 500 m Rasterauflösung ist (Myneni et al. 2015). Die maximalen und minimalen Monatsmittelwerte wurden dabei für den Zeitraum 01.08.2002 bis 01.01.2020 unter Anwendung eines Wolkenfilters berechnet. Das maximale Monatsmittel von LAI (Abb. 9a, „lai_max“ in Tab. 9 im Anhang) und auch die Differenz zwischen
Maximum und Minimum (Abb. 9d, "lai_diff“) weisen einen räumlichen Zusammenhang mit dem Waldanteil (Abb. 8c; $\mathrm{R}=0,76$ bzw. 0,75) auf. $\mathrm{LAI}_{\text {diff }}$ zeigt nahezu über das gesamte Projektgebiet nahezu idente Werte wie $\mathrm{LAI}_{\max }$. Speziell in Regionen, die von einem hohen Anteil an Nadelwald geprägt sind, sollte $\mathrm{LAI}_{\text {diff }}$ aufgrund der dauerhaften Begrünung kleiner als LAI $\max$ sein. Daher muss davon ausgegangen werden, dass das minimale Monatsmittel von LAI speziell in Regionen mit einem hohen Anteil an Nadelwald nicht korrekt erfasst wird. Schneebedeckung während der Wintermonate könnte eine mögliche Ursache für die nicht repräsentative Messung der minimalen LAIWerte sein.

Der NDVI wird aus der Rückstreuung von 2 verschiedenen Spektralbändern abgeleitet und häufig für die fernerkundungsbasierte Vegetationsüberwachung und -klassifikation (horizontale Dichte, Typ und physiologischer $\mathrm{Zu}$ stand) verwendet. Der maximale und minimale monatliche NDVI basiert auf dem Datensatz „MODIS MOD09Q1“ mit einer zeitlichen Auflösung von 8 Tagen sowie einer räumlichen Auflösung von $250 \mathrm{~m}$ (Vermote 2015). Die Berechnung erfolgte für den Zeitraum 01.04.2000 bis 01.01.2020, ebenfalls unter Filterung von wolkenbehafteten
Satellitenbildern. Zwischen dem maximalen monatlichen NDVI (Abb. 9b, „ndvi_max“) bzw. dem minimalen monatlichen NDVI (Abb. 9e, „ndvi_min“) und der mittleren Einzugsgebietshöhe (Abb. 5b) ist eine negative Korrelation $(\mathrm{R}=-0,78$ bzw. $-0,84)$ erkennbar.

Der GVF (Green Vegetation Fraction) beschreibt jenen Anteil des Bodens, der von grüner Vegetation bedeckt ist. Dieser kann dabei aus dem NDVI bei verschiedenen Gegebenheiten nach Formel (1) abgeleitet werden (Broxton et al. 2014):

$$
G V F=\frac{N D V I-N D V I_{s}}{N D V I_{c, v}-N D V I_{s}}
$$

wobei NDVI das (maximale oder minimale) Monatsmittel, $\mathrm{NDVI}_{\mathrm{s}}$ das jährliche Maximum von unbewachsenem Boden und $\mathrm{NDVI}_{\mathrm{c}, \mathrm{v}}$ das jährliche Maximum von bewachsener Bodenoberfläche in Abhängigkeit von der IGBPLandklasse darstellt (Tab. 1 in Broxton et al. 2014). $\mathrm{NDVI}_{\mathrm{s}}$ wurde dabei in Anlehnung an Broxton et al. (2014) mit dem Wert 0,09 festgelegt, während die räumliche Verteilung der IGBPLandklassen dem Datensatz „MODIS MCD12Q1“ des Jahres 2012 entnommen wurde (Friedl und Sulla-Menashe 2019). Da die Werte für $\mathrm{NDVI}_{\mathrm{s}}$ und $\mathrm{NDVI}_{\mathrm{c}, \mathrm{v}}$ für einen globalen Maßstab abgeleitet wurden und somit nicht immer 

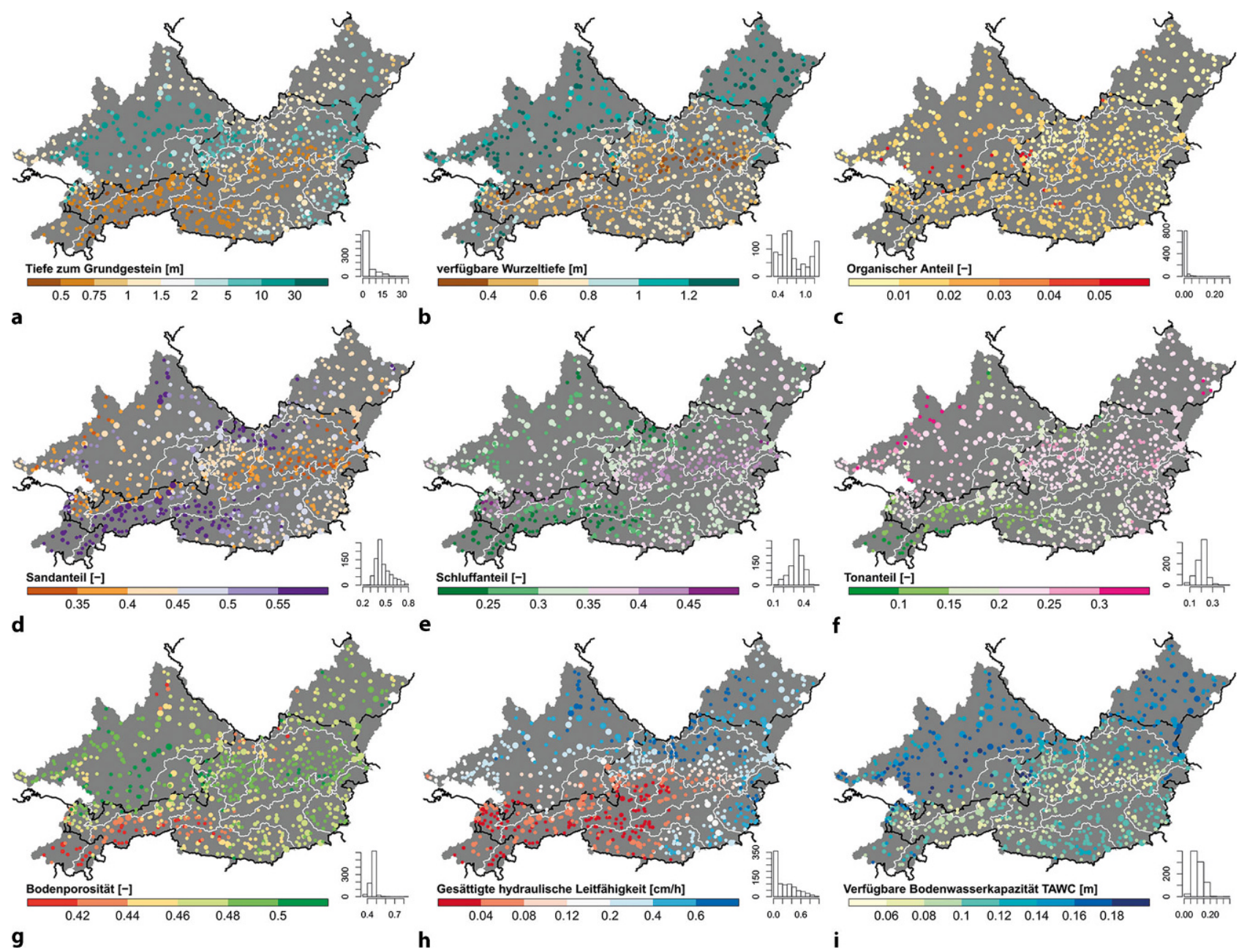

Abb. 10 Übersicht der Bodenattribute, welche die Eigenschaften des gesamten orographischen Einzugsgebiets darstellen (Einzugsgebietsabgrenzung A, Abb. 2a). Die Größe der Kreise ist dabei proportional zur Einzugsgebietsfläche. Das Histogramm zeigt die Verteilung der 859 Attributswerte an

den Bedingungen im Projektgebiet entsprechen, können die Werte für GVF auch außerhalb des Normbereiches zwischen 0 und 1 liegen. Um die Konsistenz zu wahren, wurde die GVF jedoch nicht auf den Normbereich beschränkt. Die räumliche Verteilung des maximalen Monatsmittels von GVF (Abb. 9c, "gvf_max“) weist ähnliche Muster wie jene der maximalen Monatsmittel von LAI $(R=0,79)$ sowie NDVI $(R=0,94)$ auf, während die Differenz aus maximalem und minimalem Monatsmittel von GVF (Abb. 9f, „gfv_diff“) in jenen Regionen mit einem hohen Schneeanteil (Abb. 6g) auch tendenziell höher ist.

\subsection{Bodenattribute}

LamaH beinhaltet 10 verschiedene Attribute zur Charakterisierung der Bodeneigenschaften (Tab. 10 im Anhang), wobei 8 vom Datensatz „European Soil Database Derived Data" (ESDD, Hiederer 2013a, b) abgeleitet sind. Der Datensatz mit einer Rastergröße von $1 \mathrm{~km}$ basiert wiederum auf der European Soil Database (ESD, Panagos et al. 2012; Panagos 2006), während der maximal verfügbare Bodenwassergehalt (TAWC) bei ESDD mittels Pedotransfer-Funktionen berechnet wurde (Hiederer 2013a). ESDD stellt Bodenattribute für eine Ober- und eine Unterbodenschicht mit der Grenze bei $30 \mathrm{~cm}$ Bodentiefe bereit. Daher wurden die Attribute aus diesen beiden Schichten zuerst nach der verfügbaren Wurzeltiefe „root_dep“ gewichtet aggregiert oder bei TAWC auch aufsummiert. Das Attribut, welches die Tiefe zum Festgestein beschreibt „bdrk_dep“, baut auf dem Layer „average soil and sedimentary-deposit thickness“ des Datensatzes „Global 1-km
Gridded Thickness of Soil, Regolith, and Sedimentary Deposit Layers" von Pelletier et al. (2016) auf. Dieser Datensatz weist eine räumliche Auflösung von 30 Bogensekunden (ca. $1 \mathrm{~km}$ ) auf und basiert auf landformspezifischen Modellen (für Hochland, Tiefland, Hang und Talboden) unter Berücksichtigung geomorphologischer Prinzipien, sowie unter Einbeziehung von Daten zur Topographie, zum Klima und zur Geologie. Eine Kalibrierung und Validierung erfolgte dabei anhand unabhängiger Bohrprofile (Pelletier et al. 2016). Der Datensatz „3D Soil Hydraulic Database of Europe“ (3DSHD, Toth et al. 2017) mit einer Rastergröße von $250 \mathrm{~m}$ diente als Ausgangsbasis für die Ableitung der gesättigten hydraulischen Bodenleitfähigkeit, welche mittels PedotransferFunktionen (Toth et al. 2015) aus den Bodenattributen Sand-, Schluff-, Ton- 


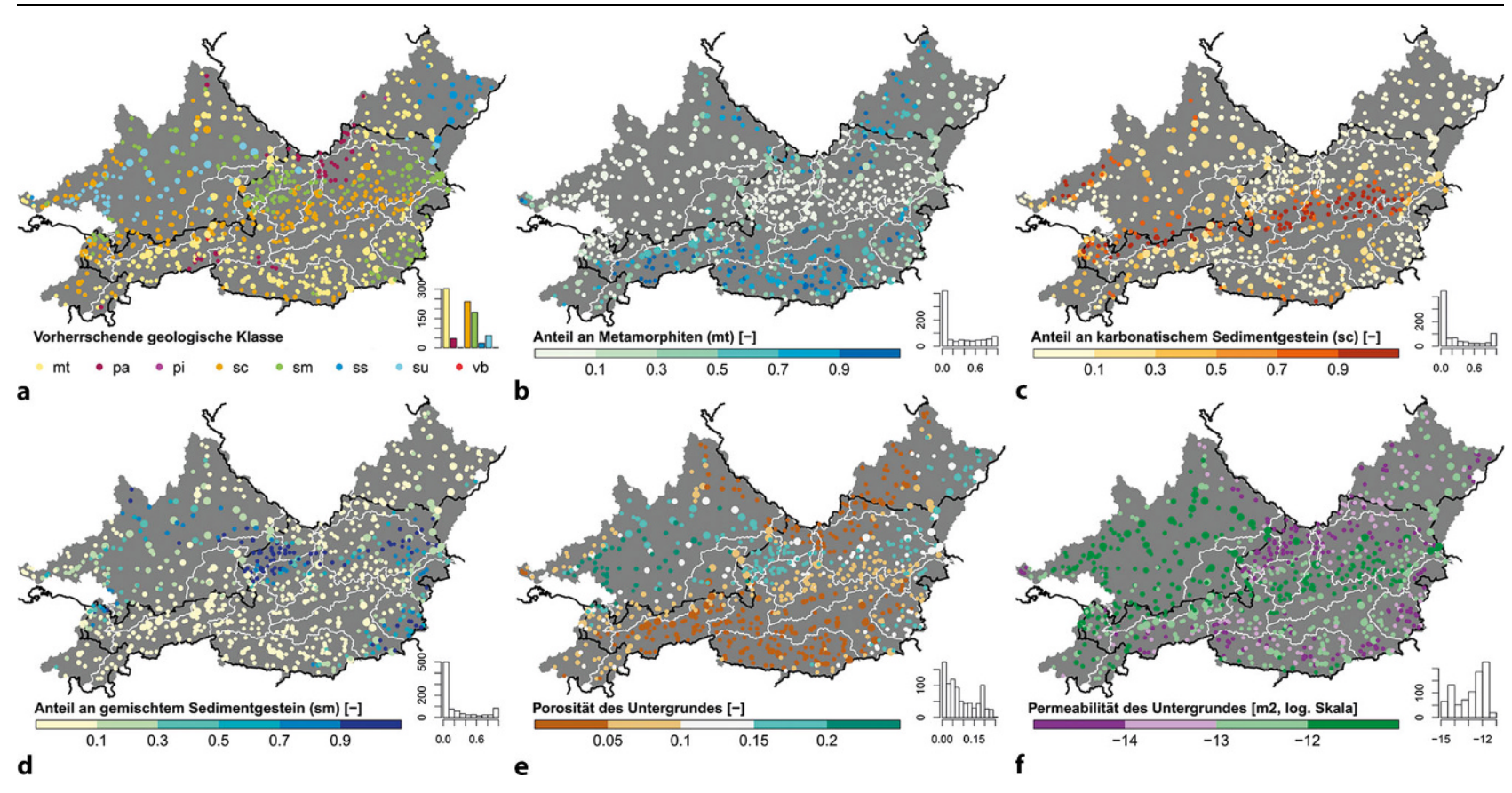

Abb. 11 Übersicht der geologischen Attribute, welche die Eigenschaften des gesamten orographischen Einzugsgebiets darstellen (Einzugsgebietsabgrenzung A, Abb. 2a). Die Größe der Kreise ist dabei proportional zur Einzugsgebietsfläche. Das Histogramm zeigt die Verteilung der 859 Attributswerte an. Klassen in a $m t$ Metamorphite, pa saure Plutonite, pi intermediäre Plutonite, $s c$ karbonatisches Sedimentgestein, sm gemischtes Sedimentgestein, ss siliklastisches Sedimentgestein, su unverfestigte Sedimente, $v b$ basische Vulkanite

anteil sowie organischem Kohlenstoffgehalt des Datensatzes „SoilGrids250m“ abgeleitet wurden. SoilGrids250m beruht wiederum auf maschinellen Lernverfahren unter Einbeziehung von Daten aus etwa 150.000 Bodenprofilen sowie Fernerkundungsdaten zu Klima, Vegetation, Geomorphologie und Lithologie (Hengl et al. 2017). Da innerhalb 3DSHD Daten für 7 verschiedene Bodenschichten zur Verfügung gestellt werden, erfolgte vor der flächenhaften Aggregierung eine tiefengewichtete harmonische Mittelung.

Die bereitgestellten Bodenattribute in LamaH beinhalten teilweise große Unsicherheiten und sollten aus mehreren Gründen mit Vorsicht betrachtet werden. Erstens basieren die Bodenattribute der ESD hauptsächlich auf extrapolierten Beobachtungen von Bodenprofilen sowie auf Expertenschätzungen (ESDB 2004). Speziell bei heterogenen Bodenverhältnissen und großen Abständen der Bodenprofile muss die Repräsentativität des Datensatzes in Frage gestellt werden. Daten aus Bodenprofilen sind auch bei 3DSHD (Hengl et al. 2017; Toth et al. 2017) und dem Datensatz von Pelletier et al. (2016) integriert, werden dort aber eher zur Kalibrierung und Validierung ver- wendet. Toth et al. (2017) weisen für 3DSHD erhöhte Unzuverlässigkeit ab $1000 \mathrm{~m}$ Seehöhe aus (ca. $24,2 \%$ des Projektgebiets liegen über $1000 \mathrm{~m} \mathrm{Mh}$ ). Weiters ist die Begrenzung der Bodentiefe bei $1,5 \mathrm{~m}$ bei ESDD sowie $2,0 \mathrm{~m}$ bei 3DSHD eine weitere Unsicherheitsquelle (Boer-Euser et al. 2016). Als letzter Punkt muss auch erwähnt werden, dass durch die Aggregierung auf Einzugsgebietsskala viel räumlich verteilte Information verloren geht.

Die Tiefe bis zum Grundgestein (Abb. 10a, „bedrk_dep“ in Tab. $10 \mathrm{im}$ Anhang) zeigt ähnliche räumliche Muster wie das mittlere Einzugsgebietsgefälle

(Abb. $5 d$; $R=-0,56$ ) sowie die mittlere Höhenlage (Abb. 5b; $R=-0,46$ ). Etwa $37 \%$ aller 859 Einzugsgebiete haben eine mittlere Tiefe bis zum Grundgestein von mehr als $1,5 \mathrm{~m}$. Diese Tiefe entspricht dem Maximum der wurzelverfügbaren Tiefe in ESDD (Abb. 10b, „root_dep“). Die für Wurzeln verfügbare Tiefe ist in Deutschland und der Tschechischen Republik tendenziell höher als in anderen Regionen. Es ist unklar, ob dies ein Hinweis auf unterschiedliche Messmethoden in den jeweiligen Ländern ist. Gering verfügbare Wurzeltiefen sind in Österreich gemäß
Abb. 10b vor allem dort vorhanden, wo der Anteil an karbonatischem Sedimentgestein (Abb. 11c) oder Gletschern (Abb. 8d) hoch ist. $40 \%$ aller Einzugsgebiete weisen einen mittleren organischen Bodengehalt unter $1 \%$ auf, wobei die höchsten org. Bodenanteile nach Aggregierung auf Einzugsgebietsebene im süddeutschen Raum vorhanden sind (Abb. 10c, „oc_fra“). Hinsichtlich der verschiedenen Korngrößenfraktionen sind Zusammenhänge mit dem Grundgestein erkennbar: 1) Ein hoher Sandanteil (Abb. 10d, „sand_fra“) ist besonders dort vorherrschend, wo auch der Anteil an metamorphem Grundgestein hoch ist (Abb. 11b; $R=0,47$ ). 2) Weiters ist der Anteil an Schluff (Abb. 10e, „silt_fra“) tendenziell hoch, wo ein hoher Anteil an karbonatischem Sedimentgestein (Abb. 11c; R=0,52) vorhanden ist. 3) Schließlich ist eine Zunahme des Tonanteils (Abb. 10f, "clay_fra“) mit zunehmendem Anteil an gemischtem Sedimentgestein (Abb. 11d, $R=0,47$ ) beobachtbar. Die Bodenporosität (Abb. 10g, „,soil_poros“) weist ähnliche räumliche Muster wie jene des Sandanteils auf $(R=-0,79)$, während die gesättigte hydraulische Leitfähigkeit (Abb. 10h, ,soil_condu“) mit abnehmender mittlerer Einzugsgebiets- 


\begin{tabular}{|c|c|c|c|}
\hline Attribut & Beschreibung & Einheit & Datengrundlage \\
\hline $\begin{array}{l}\text { typim- } \\
\text { pact }\end{array}$ & $\begin{array}{l}\text { Arten der Pegelbeeinflussung: } \\
\text { A - Wasserreservoir mit ganzjähriger Füllung } \\
\text { B - Hochwasserrückhaltebecken } \\
\text { C - See mit steuerbarem Auslass (z.B. Wehr) } \\
\text { D - See mit unbeeinflusstem Abfluss } \\
\text { E - Wasserentnahmen } \\
\text { F - Not oder Grundablass eines Wasserreservoirs } \\
\text { G - Extremwerte sind beeinflusst bzW. werden nur unzureichend } \\
\text { genau gemessen (z. B. Ausuferungen) } \\
\text { H - künstlicher Kanal } \\
\text { I - Wasserein- bzW. Überleitung (von anderem Gewässer oder } \\
\text { Einzugsgebiet) } \\
\text { J - Verkrautung am Pegel } \\
\text { K - Fischteiche } \\
\text { L - starke Infiltration } \\
\text { M - (Karst-) Quelle }\end{array}$ & - & $\begin{array}{l}\text { BAFU (2020), } \\
\text { CHMI (2020), } \\
\text { GKD (2020), } \\
\text { HZB (2020), } \\
\text { LUBW (2020) }\end{array}$ \\
\hline diur_art & $\begin{array}{l}\text { Unnatürliche systematische Schwankungen im Hydrographen } \\
\text { mit stündlicher Auflösung, binäre Klassifikation ( } 0 \text { - nein, } 1 \text { - ja) }\end{array}$ & - & Siehe oberhalb \\
\hline diur_glac & $\begin{array}{l}\text { Systematische Schwankungen im Hydrographen mit stündli- } \\
\text { cher Auflösung, welche auf Gletscher- oder Schneeschmelze } \\
\text { zurückzuführen sind, binäre Klassifikation }(0-\text { nein, } 1 \text { - ja) }\end{array}$ & - & Siehe oberhalb \\
\hline $\begin{array}{l}\text { degim- } \\
\text { pact }\end{array}$ & $\begin{array}{l}\text { Grade der Pegelbeeinflussung: } \\
\mathrm{u} \text { - unbeeinflusst } \\
\text { I- gering beeinflusst } \\
\mathrm{m} \text { - moderat beeinflusst } \\
\mathrm{s} \text { - stark beeinflusst } \\
\mathrm{x} \text { - nicht weiter berücksichtigt (hauptsächlich Pegel mit unkla- } \\
\text { rem Einzugsgebiet, z. B. künstliche Kanäle oder Karstquellen) }\end{array}$ & - & Siehe oberhalb \\
\hline
\end{tabular}

höhe (Abb. 5b, R=-0,63) tendenziell zunimmt. Die verfügbare Bodenwassertiefe (TAWC, „soil_tawc") wurde beim verwendeten Ausgangsdatensatz ESDD unter Einbeziehung des Wassergehalts bei Feldkapazität, des Kiesanteils sowie der wurzelverfügbaren Tiefe berechnet (Hiederer 2013a). Damit kann auch der deutliche Zusammenhang von TAWC (Abb. 10i) mit der wurzelverfügbaren Bodentiefe $(\mathrm{R}=0,94)$ erklärt werden.

\subsection{Geologische Attribute}

Für die Ableitung von 16 verschiedenen geologischen Attributen (Tab. $11 \mathrm{im}$ Anhang) wurden mit GLiM (Hartmann und Moosdorf 2012, Global Lithological Map) und GLHYMPS (Gleeson et al. 2014, Global Hydrogeology MaPS) zwei globale Datensätze herangezogen.

Die Anteile an verschiedenen geologischen Klassen basieren auf GLiM, welcher 92 regionale geologische Karten zusammenfasst und in vektorieller Form zur Verfügung stellt. GLiM bietet 3 Detailstufen an, wobei die 1 . Stufe die dominante lithologische Klasse indiziert, während die optionale 2. sowie 3. Stufe unter anderem das Gefüge des Gesteins sowie lokale Gegebenheiten näher spezifiziert (Hartmann und Moosdorf 2012). Bei LamaH wurde nur die 1. Stufe von GLiM herangezo- gen, welche 16 verschiedene Klassen beinhaltet. Die Klassen der 1. Stufe „Evaporite“, „keine Daten“ sowie „intermediäre Vulkanite" kommen innerhalb des Projektgebiets nicht vor, daher weist Tab. 11 nur Anteile für 13 verschiedene Klassen auf. Die 3 häufigsten dominanten geologischen Klassen (Abb. 11a, „gc_dom“ in Tab. 11 im Anhang) innerhalb des Projektgebiets und über alle 859 Einzugsgebiete sind Metamorphite ( $m t 35,1 \%$ ), karbonatisches Sedimentgestein ( $s c 27,4 \%$ ), sowie gemischte Sedimentgesteine ( $s m 21,2 \%$ ). Metamorphe Gesteine ( $\mathrm{mt}$ ) sind vor allem entlang der nördlichen Grenze des Projektgebiets (Böhmische Masse) sowie im südlicheren Projektgebiet (Zentrale Ostalpen) vorherrschend (Abb. 11b, „gc_mt_fra“) und inkludieren dabei hauptsächlich Schiefer, Gneis und Quarzit. Aus hydrologischer Sicht ist vor allem der Anteil an karbonatischem Sedimentgestein (sc) interessant, da ein hoher Anteil ein Indikator für Karstsysteme sein kann. Hohe Anteile finden sich vor allem entlang des Gürtels vom Südwesten zum zentralen Osten (Nördliche Kalkalpen), der zentralen Südgrenze (Südliche Kalkalpen) sowie der nordöstlichen Grenze (Schwäbische Alb) des Projektgebietes (Abb. 11c, „gc_sc_fra“). Die Flyschund Molassezone (Alpenvorland und zentrale Teile des deutschen Projektgebiets) ist grundsätzlich durch einen hohen Anteil an gemischten Sedimentgesteinen (sm) gekennzeichnet (Abb. 11d, „gc_sm_fra“).

Die Attribute bezüglich Permeabilität und Porosität des lithologischen Untergrunds wurden von GLHYMPS abgeleitet, wobei die hydraulischen Eigenschaften anhand der lithologischen Klassen von GLiM definiert worden sind. GLHYMPS ist dabei nur für Anwendungen auf regionaler Ebene gedacht (d.h. räumliche Auflösung ab $5 \mathrm{~km}$ ), da ab dieser Skala der Einfluss von lokalen Heterogenitäten wie Störungszonen vernachlässigt werden kann (Gleeson et al. 2014). Zudem wird die Permeabilität nur für gesättigte Bedingungen determiniert (Huscroft et al. 2018). Ein hoher Anteil an Metamorphiten (mt) oder Plutoniten (pa, pi) ist in Abb. 1la oft mit einer geringen Porosität (Abb. 11e, „geol_poros“) einhergehend. Dies ist aufgrund der Konnektivität der beiden Datensätze GLiM und GLHYMPS jedoch wenig überraschend. Im Gegensatz dazu weisen Regionen innerhalb der Flysch- und Molassezone eine relativ hohe Untergrundporosität auf. Eine relativ hohe Untergrundporosität muss nicht zwangsläufig mit einer hohen Untergrundpermeabilität („geol_perme“) einhergehen, daher ist das räumliche Muster in Abb. 11f inhomogener als in Abb. 1le. Begründet werden kann dies mit dem Gesteinsgefüge (abgeleitet aus der 2. Stufe von GLiM), welches unterschiedlich starke Auswirkungen auf Permeabilität und Porosität haben kann (Tab. 1 in Gleeson et al. 2014).

\subsection{Beeinflussung des \\ Abflussgeschehens in den Einzugsgebieten und in der Pegelmessung}

Um die Filterung und Evaluation der Abflusspegel hinsichtlich einer etwaigen (anthropogenen) Beeinflussung des Abflussgeschehens oder dessen Messung zu vereinfachen, werden 4 Attribute spezifiziert (Tab. 2). Die Art der Beeinflussung („typimpact“) wurde in erster Linie aus Pegel-Zusatzinformationen in den Metadaten seitens der betreffenden hydrographischen bzw. gewässerkundlichen Dienste (BAFU 2020; GKD 2020; HZB 2020; LUBW 2020) bestimmt. Für die Zuteilung wurden aber auch öffentlich zugängliche Informationen sowie manuelle Luft- 


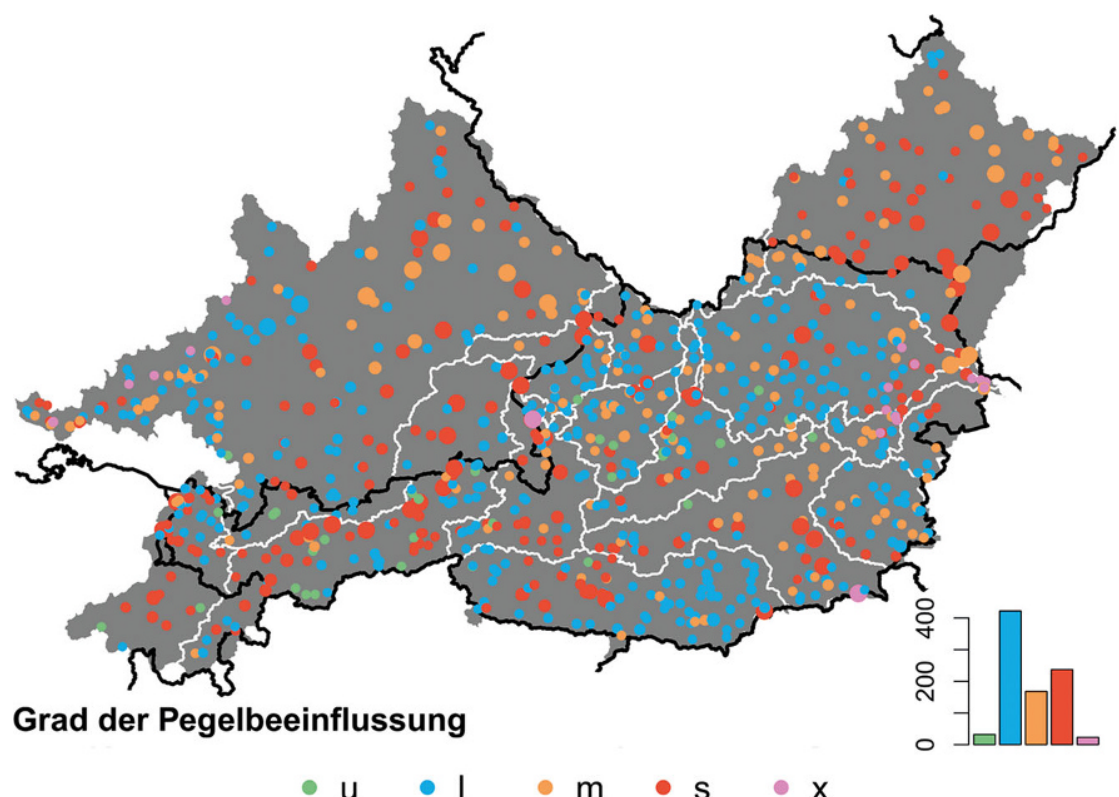

Abb. 12 Grad der Pegel- bzw. Einzugsgebietsbeeinflussung. Die Größe der Kreise ist dabei proportional zur Einzugsgebietsfläche. Das Histogramm zeigt die Verteilung der 882 Attributswerte an. Grade der Beeinflussung: $u$ unbeeinflusst, / gering beeinflusst, $m$ moderat beeinflusst, $s$ stark beeinflusst, $x$ nicht weiter berücksichtigt (hauptsächlich Pegel mit unklarem Einzugsgebiet, z. B. künstliche Kanäle oder Karstquellen)

bildauswertungen herangezogen. Die 13 verschiedenen Arten der Pegelbeeinflussung sind in der Beschreibung der Tab. 2 erläutert. Typische anthropogene Beeinflussungen innerhalb LamaH stellen große Wasserreservoirs dar, welche oft mit Wasserkraftwerken und einzugsgebietsübergreifenden Wassertransfers einhergehen. Folgende Beeinflussungsarten wurden nicht klassifiziert, da die notwendigen Informationen dazu nicht oder nur mit sehr großem Aufwand konsistent erhältlich sind: 1) Vereisung, speziell an kleineren Bächen im Winter; 2) veränderliche Gerinneprofile, die $\mathrm{zu}$ ungenauen Wasserstands-AbflussBeziehungen führen; 3) hoher Grundwasserbegleitstrom im Umfeld des Pegels sowie 4) unterirdische $\mathrm{Zu}$ - oder Abflüsse speziell in stark verkarstetem Gebiet.

Ergänzend wurden die Hydrographen mit stündlicher Auflösung der Monate Januar und Juli in den Jahren 1990, 2005 und 2017 visuell in Hinblick auf systematische Tagesschwankungen beurteilt („diur_art“/,diur_glac“). Dabei erfolgte weiters eine Unterteilung in künstlich (z.B. durch Speicheroder Laufwasserkraftwerke mit Schwellbetrieb, Kläranlagen) sowie natürlich (Schnee- oder Gletscherschmelze) verursachte Schwankungen (Tab. 2). Der Grad der Pegelbeeinflussung („deg- impact") wird anhand von 5 Klassen (Tab. 2) meist auf Basis der Beeinflussungsart sowie etwaiger systematischer Tagesschwankungen festgelegt. Ein geringer Grad $(l)$ wurde jenen Pegeln zugeteilt, welche von der Beeinflussungsart D (See mit unbeeinflusstem Abfluss) oder J (Verkrautung am Pegel) geprägt sind. Seen können speziell im Hochwasserfall als Retentionsraum wirken und damit eine Dämpfung sowie Verzögerung des Hochwasserscheitelwerts zur Folge haben. Ein moderater Grad $(m)$ wurde für die Beeinflussungsarten B (Hochwasserrückhaltebecken), C (See mit steuerbarem Auslass), F (Not- oder Grundablass eines Wasserreservoirs), G (Extremwerte sind beeinflusst bzw. werden nur unzureichend genau gemessen), K (Fischteiche) sowie $\mathrm{L}$ (hohe Infiltration) vergeben. Eine Ausnahme wurde dabei für 3 Pegel an der oberen Donau gemacht, welche in den Sommermonaten stark $(s)$ von Vollversickerung betroffen sein können (Hötzl 1996). Pegeln mit der Beeinflussungsart A (Wasserreservoir mit ganzjähriger Füllung) wurde in den meisten Fällen ein starker $(s)$ und wenigen Pegeln mit sehr großem Einzugsgebiet (ausgewählte Donaupegel) ein moderater $(m)$ Beeinflussungsgrad zugeordnet. Schließlich erfolgte eine ausmaßbasierte $\mathrm{Zu}$ weisung des Beeinflussungsgrads im
Falle von Beeinflussungsart E (Wasserentnahmen) oder I (Wassereinleitung). Im Hydrographischen Jahrbuch von Österreich sind anthropogene, einzugsgebietsübergreifende Wassertransfers über die Vergrößerung oder Verkleinerung der natürlichen Einzugsgebietsgröße eines Pegels deklariert (BMLFUW 2013). Mit dieser Information wurde für Pegel auf österreichischem Territorium eine relative anthropogene Veränderung der Einzugsgebietsgröße berechnet, die eine nachvollziehbare und damit objektive Einteilung erlaubt. Keine Beeinflussung $(u)$ wurde bei einer künstlichen Änderung der Einzugsgebietsgröße unter $1 \%$ zugeteilt, während im Falle einer Änderung zwischen 1 und $3 \%$ ein geringer Grad $(l)$ der Beeinflussung vermerkt wurde. Weiters erfolgte bis $\mathrm{zu}$ einer Änderung von $10 \%$ eine Zuteilung eines moderaten $(m)$, sowie bei über $10 \%$ die Zuteilung eines starken Beeinflussungsgrads $(s)$. Bei Pegeln außerhalb Österreichs gibt es keine Information bezüglich der künstlichen Einzugsgebietsgrößenänderung. Daher wurde dort, und auch bei österreichischen Pegeln welche von anderen Arten der Wasserentnahme (Flussverzweigungen, Ausleitungen oder Bewässerungsentnahmen) beeinflusst sind, der Beeinflussungsgrad anhand öffentlich zugänglicher Informationen sowie Luftbildanalysen ermittelt. Dabei wurde meist ein starker $(s)$, in ein paar Fällen (z. B. Entnahmen für Trinkwasser) auch ein moderater $(m)$ Beeinflussungsgrad zugeordnet. Systematische Tagesschwankungen künstlichen Ursprungs (z. B. Schwellbetrieb) wurden als „stark beeinflusst“ $(s)$ definiert („diur_art“ in Tab. 2). Ein Pegel bzw. Einzugsgebiet kann selbstverständlich auch von mehreren Beeinflussungsarten geprägt sein. In diesem Fall wird der höchste Beeinflussungsgrad angenommen. Falls ein Pegel in oder unterhalb besiedelten Gebiets liegt und sonst keiner anderen Beeinflussungsart unterliegt, kam pauschal ein geringer Grad $(l)$ der Beeinflussung zur Anwendung. Grund dafür ist eine mögliche Beeinflussung durch Wasserentnahmen oder Regenwasserkanäle, welche bei der Deklaration der Beeinflussungsart jedoch nicht berücksichtigt wurde. Wenn keine offensichtliche Beeinflussung vorliegt und der Pegel oberhalb von besiedeltem Gebiet liegt, wurde der Grad als unbeeinflusst $(u)$ deklariert. Abschließend ist der Beeinflussungsgrad $(x)$ für all jene Pegel definiert worden, welche 
1) kein eindeutig zuordenbares Einzugsgebiet haben (z.B. Pegel an künstlichem Gerinne (Beeinflussungsart $\mathrm{H}$ ) oder unterhalb Karstquelle), 2) mehrere Zeitreihen zugeordnet sind (z.B. mit oder ohne Berücksichtigung von Mühlkanälen) sowie 3) zu viele Lücken in der Zeitreihe (>50\%) aufweisen. Diesen Pegeln sind in weiterer Folge keine meteorologischen Zeitreihen oder Einzugsgebietseigenschaften aufgrund fehlender Einzugsgebietsabgrenzung zugewiesen worden.

Die räumliche- als auch die Häufigkeitsverteilung des Pegelbeeinflussungsgrads ist in Abb. 12 dargestellt. Von 882 Pegeln sind nach Kategorisierung $3,5 \%$ unbeeinflusst $(u), 48 \%$ gering $(l), 18,9 \%$ moderat $(m)$ sowie $27 \%$ stark beeinflusst $(s)$, während $2,6 \%$ der Klasse $(x)$ zugehörig sind. Gering beeinflusste Pegel $(l)$ sind vor allem im Nordwesten des deutschen Projektgebiets, im Norden des österreichischen Zentralraums (Flussgebiet 5, 6, 7, 8, 9, 10 in Abb. 1), aber auch im Osten (Mitte des Flussgebietsgebiets 16) sowie Süden Österreichs (Osten des Flussgebiets 18) vorherrschend. Stark beeinflusste Pegel $(s)$ sind dagegen vor allem dort vorhanden, wo große Wasserspeicher primär zur Energieerzeugung (vor allem im Alpenraum), aber auch zum saisonalen Wasserausgleich oder Hochwasserschutz (vor allem in Tschechien und im Norden des deutschen Projektgebiets) in Betrieb sind. $\mathrm{Zu}$ beachten ist, dass auch weit von großen Wasserspeichern stromab gelegene Pegel noch stark von diesen beeinflusst sein können.

\section{Zusammenfassung}

Hydrologische Untersuchungen sind meist stark von umfangreichen Datengrundlagen abhängig. Bei großräumigen oder länderübergreifenden Projekten ist und war es daher oft mühselig und zeitaufwendig, die benötigten Daten zu sammeln und anschließend die meist unterschiedlichen Formate, Definitionen und Konventionen zu homogenisieren. Dies liegt unter anderem an unterschiedlichen Organisationsformen der hydrographischen Behörden, Kommunikationsbarrieren und auch Misstrauen (wobei dieses, zumindest in Europa aufgrund der europäischen Integration, nennenswert abgenommen hat). Mit dem Datensatz LamaH wird eine einzigartige, homogene Datengrundlage für hydrologische, aber auch andere umweltwissenschaftliche Disziplinen bereitgestellt, mit dem die bisherigen Hemmnisse überwunden werden können. LamaH umfasst neben dem vollständigen Territorium Österreichs auch alle benachbarten Oberliegergebiete der durch Österreich fließenden Gewässer und stellt somit eine Initiative für einen umfassenden, länderübergreifenden hydrologischen Datensatz dar. LamaH beinhaltet neben Abflusszeitreihen auch 15 meteorologische Zeitreihen (tägliche und stündliche Auflösung) sowie über 60 Attribute für 859 Einzugsgebiete. Die 3 verschiedenen Einzugsgebietsabgrenzungen ermöglichen Untersuchungen für einzelne unabhängige Einzugsgebiete, aber auch innerhalb eines zusammenhängenden Flussnetzes unter Berücksichtigung von Zwischeneinzugsgebieten. Aufgrund der Vielzahl an einbezogenen Datenquellen beinhaltet LamaH klarerweise auch Defizite und Unsicherheiten, wobei aber versucht wurde diese zu erläutern.

Blöschl et al. (2019b) verweisen auf zahlreiche ungelöste Aufgaben in der Hydrologie, wie beispielsweise die Abflussprognose in unbeobachteten Einzugsgebieten (PUB). Für die Frage der reinen Zeitreihenvorhersage (z.B. Kratzert et al. 2019a, b, 2018) zeigen Methoden, welche auf maschinellen Lernverfahren („machine learning“) basieren, vielversprechende Ergebnisse. Hilfreich für die Anwendung dieser datengetriebenen Methoden sind jedoch einheitlich aufgebaute "large-sample“Datensätze, da einerseits die notwendige Vorarbeit drastisch reduziert und andererseits der Austausch bzw. die Vergleichbarkeit der Modellierungsergebnisse wesentlich erleichtert wird. In Anbetracht des Umfangs von LamaH hoffen wir, dass dieser Datensatz als solide Datenbasis für weitere Untersuchungen in verschiedenen Bereichen der Hydrologie und benachbarter Felder der Umweltwissenschaften dienen wird. Die hohe Variabilität in den Daten könnte in Kombination mit dem zusammenhängenden Flussnetzwerk sowie der hohen zeitlichen Auflösung der Zeitreihen ein verbessertes Prozessverständnis für Wassertransfer und -speicherung gewähren, sofern auch geeignete Methoden verwendet werden.

Danksagung Wir möchten den gewässerkundlichen und hydrographischen Diensten der (Bundes-)Länder
Baden-Württemberg, Bayern, Österreich, Schweiz sowie Tschechien für die Bereitstellung der Abflusszeitreihen herzlich danken. Dank gilt auch Frederik Kratzert, der den Datensatz vorab getestet hat und uns so einige Verbesserungsmöglichkeiten vorschlagen konnte. Die Datenverarbeitung wurde mit den frei verfügbaren Softwarepaketen R (R Core Team 2020), Python (Python Software Foundation 2020) und QGIS (QGIS Development Team 2020) durchgeführt. Besonderer Dank gebührt daher all jenen, die die zahlreichen Open-Source-Softwarepakete und -Erweiterungen entwickelt haben oder die ihre Erfahrungen diesbezüglich in den zahlreichen Online-Foren mit anderen teilen. LamaH baut auf frei verfügbaren Daten auf und wäre daher ohne die Institutionen, Arbeitsgruppen und Personen, welche an den verwendeten Datengrundlagen teilweise über Jahre gearbeitet haben, nicht möglich gewesen.

Funding Open access funding provided by University of Natural Resources and Life Sciences Vienna (BOKU).

Open Access Dieser Artikel wird unter der Creative Commons Namensnennung 4.0 International Lizenz veröffentlicht, welche die Nutzung, Vervielfältigung, Bearbeitung, Verbreitung und Wiedergabe in jeglichem Medium und Format erlaubt, sofern Sie den/die ursprünglichen Autor(en) und die Quelle ordnungsgemäß nennen, einen Link zur Creative Commons Lizenz beifügen und angeben, ob Änderungen vorgenommen wurden.

Die in diesem Artikel enthaltenen Bilder und sonstiges Drittmaterial unterliegen ebenfalls der genannten Creative Commons Lizenz, sofern sich aus der Abbildungslegende nichts anderes ergibt. Sofern das betreffende Material nicht unter der genannten Creative Commons Lizenz steht und die betreffende Handlung nicht nach gesetzlichen Vorschriften erlaubt ist, ist für die oben aufgeführten Weiterverwendungen des Materials die Einwilligung des jeweiligen Rechteinhabers einzuholen.

Weitere Details zur Lizenz entnehmen Sie bitte der Lizenzinformation auf http://creativecommons.org/licenses/ by/4.0/deed.de. 


\section{Anhang}

Tab. 3 Abflusspegel-bezogene Attribute

\begin{tabular}{|c|c|c|c|}
\hline Attribut & Beschreibung & Einheit & Datengrundlage \\
\hline ID & $\begin{array}{l}\text { ID Nummer, die gleiche ID ist auch dem zugehörigen Einzugsgebiet zugeord- } \\
\text { net, dieses Attribut ermöglicht also die Verlinkung zwischen Abflusszeitreihe, } \\
\text { meteorologischer Zeitreihen und den Einzugsgebietseigenschaften }\end{array}$ & - & - \\
\hline govnr & Offizielle Pegelnummer, definiert von der zugehörigen staatlichen Institution & - & $\begin{array}{l}\text { BAFU (2020), CHMI (2020), GKD (2020), } \\
\text { HZB (2020), LUBW (2020) }\end{array}$ \\
\hline name & Offizieller Pegelname & - & Siehe oberhalb \\
\hline river & Name des gemessenen Gewässers & - & Siehe oberhalb \\
\hline area_gov & Einzugsgebietsgröße, welche in den Metadaten der Pegel angegeben ist & $\mathrm{km}^{2}$ & Siehe oberhalb \\
\hline elev & Pegelnullpunkt, welcher in den Metadaten der Pegel angegeben ist & $\mathrm{m} \mathrm{Mh}$ & Siehe oberhalb \\
\hline Ion & Geogr. Länge im System LAEA Europe (EPSG 3035) & $\mathrm{m}$ & Siehe oberhalb \\
\hline lat & Geogr. Breite im System LAEA Europe (EPSG 3035) & $\mathrm{m}$ & Siehe oberhalb \\
\hline country & ISO 3166 ALPHA-3 Ländercode & - & Siehe oberhalb \\
\hline fedstate & $\begin{array}{l}\text { Abkürzung für das Bundesland, wasserwirtschaftliche Verwaltung basiert meist } \\
\text { auf Ebene der Bundesländer }{ }^{\mathrm{a}}\end{array}$ & - & Siehe oberhalb \\
\hline region & Flussgebiet $^{\mathrm{b}}$ & - & Siehe oberhalb \\
\hline obsbeg_day & Start der tägl. Abflusszeitreihen im Datensatz & Jahr & Siehe oberhalb \\
\hline obsbeg_hr & Beginn der kontinuierlichen (stündlichen) Abflussdatenaufzeichnung & Jahr & Siehe oberhalb \\
\hline obsend & $\begin{array}{l}\text { Ende der kontinuierlichen (stündlichen) Abflussdatenaufzeichnung, tägliche } \\
\text { Aufzeichnung kann darüber hinaus noch aufrecht sein, Nummer } 0 \text { weist auf eine } \\
\text { bestehende Aufzeichnung hin }\end{array}$ & Jahr & Siehe oberhalb \\
\hline gaps_post & $\begin{array}{l}\text { Anteil der Lücken in der vorhandenen stündlichen Abflusszeitreihe nach Bear- } \\
\text { beitung (lin. Interpolation, bis zu } 6 \text { h), Lücken sind mit -999 in den Zeitreihen } \\
\text { indiziert }\end{array}$ & $\%$ & Siehe oberhalb \\
\hline area_ratio & $\begin{array}{l}\text { Verhältnis zwischen der aggregierten Einzugsgebietsfläche mit Einzugsgebiets- } \\
\text { abgrenzung A „area_calc“ (Tab. 5) sowie der offiziell angeführten Einzugsge- } \\
\text { bietsfläche „area_gov“ }\end{array}$ & - & $\begin{array}{l}\text { BAFU (2020), CHMI (2020), GKD (2020), } \\
\text { HZB (2020), LUBW (2020), HAO (2007), } \\
\text { HydroATLAS (Linke et al. 2019) }\end{array}$ \\
\hline HIERARCHY & Pegelhierarchie $^{c}$ & - & Siehe oberhalb \\
\hline NEXTUPID & $\begin{array}{l}\text { ID des nächst flussaufwärts liegenden Pegels (kann kein, ein oder mehrere } \\
\text { Pegel sein), } 0 \text { indiziert keinen flussaufwärts liegenden Pegel }{ }^{\mathrm{C}}\end{array}$ & - & Siehe oberhalb \\
\hline NEXTDOWNID & $\begin{array}{l}\text { ID des nächst flussabwärts liegenden Pegels (max. } 1 \text { Pegel), } 0 \text { indiziert keinen } \\
\text { flussabwärts liegenden Pegel }^{\mathrm{C}}\end{array}$ & - & Siehe oberhalb \\
\hline dist_hup & $\begin{array}{l}\text { Horizontale Fließdistanz vom entferntesten Beginn des Gewässernetzes inner- } \\
\text { halb des Einzugsgebiets bis zum Pegel }{ }^{d}\end{array}$ & $\mathrm{~km}$ & $\begin{array}{l}\text { BAFU (2020), CHMI (2020), GKD (2020), } \\
\text { HZB (2020), LUBW (2020), EU-Hydro - } \\
\text { River Network Database (EEA 2019) }\end{array}$ \\
\hline dist_hdn & $\begin{array}{l}\text { Horizontale Fließdistanz vom aktuellen Pegel zum nächst flussabwärts liegenden } \\
\text { Pegel }^{c}\end{array}$ & $\mathrm{~km}$ & Siehe oberhalb \\
\hline elev_diff & $\begin{array}{l}\text { Höhendifferenz zwischen dem Pegelnullpunkt des aktuellen Pegels zu jenem } \\
\text { des nächst flussabwärts liegenden Pegels }{ }^{c}\end{array}$ & $\mathrm{~m}$ & Siehe oberhalb \\
\hline strm_slope & $\begin{array}{l}\text { Durchschnittliches Gefälle zwischen aktuellem Pegel und nächst flussabwärts } \\
\text { liegendem Pegel, Quotient zwischen „elev_diff“ und „dist_hdn“ c }\end{array}$ & $\mathrm{m} \mathrm{km}^{-1}$ & Siehe oberhalb \\
\hline ckhs & $\begin{array}{l}\text { Hinweis ob der Abflusswert eines Zeitschrittes von der entsprechenden Admi- } \\
\text { nistration geprüft wurde oder nicht, das genaue Ausmaß der Überprüfung wird } \\
\text { dabei nicht definiert }^{\mathrm{e}}\end{array}$ & $\begin{array}{l}0 \text { (Nein) } \\
\text { oder } 1 \text { (Ja) }\end{array}$ & $\begin{array}{l}\text { BAFU (2020), CHMI (2020), GKD (2020), } \\
\text { HZB (2020), LUBW (2020) }\end{array}$ \\
\hline \multicolumn{4}{|c|}{$\begin{array}{l}\text { a Abkürzungen für Attribut „fedstate“: Österreich (BLD Burgenland, CRN Kärnten, LAT Niederösterreich, SBG Salzburg, STY Steiermark, TYR Tirol, UAT Ober- } \\
\text { österreich, VBG Vorarlberg, VIE Wien); Deutschland (BAV Bayern, BWT Baden-Württemberg); Schweiz (GRI Graubünden, STG Sankt Gallen); Liechtenstein } \\
\text { (LIE Liechtenstein); Tschechische Republik (OLM OImütz, SBO Südböhmen, SMO Südmähren, VYS Hochland, ZLNZlin). } \\
\text { b Abkürzungen für Attribut „region“: } 1 \text { - Rhein, 2 - Donau oberhalb Inn, } 3 \text { - Inn oberhalb Salzach, } 4 \text { - Salzach, } 5 \text { - Inn unterhalb Salzach, } 6 \text { - Donau zwischen } \\
\text { Inn und Traun, } 7 \text { - Traun, } 8 \text { - Donau zwischen Traun und Enns, } 9 \text { - Enns, } 10 \text { - Donau zwischen Enns und March, } 11 \text { - Moldau, } 12 \text { - March, } 13 \text { - Donau } \\
\text { zwischen March und Leitha, } 14 \text { - Leitha, 15 - Rabnitz, } 16 \text { - Raab, } 17 \text { - Mur, } 18 \text { - Drau } \\
{ }^{c} \text { nur für Einzugsgebietsabgrenzung B und C (Abb. 2b und c) } \\
{ }^{d} \text { nur für Einzugsgebietsabgrenzung A (Abb. 2a) } \\
\text { e }_{\text {in den tägl. und stündl. Abflusszeitreihen einsehbar }}\end{array}$} \\
\hline
\end{tabular}




\begin{tabular}{|c|c|c|c|}
\hline Variable stündlich & Aggregation tägl & Beschreibung & Einheit \\
\hline DOY & Unverändert & Tag des Jahres & - \\
\hline HOD & Entfernt & Stunde des Tages & - \\
\hline $2 \mathrm{~m} \_$temp & Max, Mittel, Min & Lufttemperatur in $2 \mathrm{~m}$ Höhe & ${ }^{\circ} \mathrm{C}$ \\
\hline $2 m \_d p \_t e m p$ & Max, Mittel, Min & Taupunkttemperatur in $2 \mathrm{~m}$ Höhe & ${ }^{\circ} \mathrm{C}$ \\
\hline 10m_wind_u & Arith. Mittel & $\begin{array}{l}\text { Horizontale Geschwindigkeit der Luft, die sich in einer Höhe von } 10 \mathrm{~m} \text { über der Erdoberfläche in Rich- } \\
\text { tung Osten bewegt }\end{array}$ & $\mathrm{m} \mathrm{s}^{-1}$ \\
\hline 10m_wind_v & Arith. Mittel & $\begin{array}{l}\text { Horizontale Geschwindigkeit der Luft, die sich in einer Höhe von } 10 \mathrm{~m} \text { über der Erdoberfläche in Rich- } \\
\text { tung Norden bewegt }\end{array}$ & $\mathrm{m} \mathrm{s}^{-1}$ \\
\hline fcst_alb & Arith. Mittel & $\begin{array}{l}\text { Albedo, Anteil der solaren (kurzwelligen) Strahlung, der von der Erdoberfläche reflektiert wird (direkt } \\
\text { und diffus) }\end{array}$ & - \\
\hline lai_high_veg & Arith. Mittel & Hälfte der gesamten grünen Blattlläche pro Einheit der horizontalen Bodenfläche für hohe Vegetation & - \\
\hline lai_low_veg & Arith. Mittel & Hälfte der gesamten grünen Blattläche pro Einheit der horizontalen Bodenfläche für niedrige Vegetation & - \\
\hline swe & Arith. Mittel & Wasseräquivalent des Schnees & $\mathrm{mm}$ \\
\hline surf_net_solar_rad & $\begin{array}{l}\text { Max. Wert, Arith. } \\
\text { Mittel }\end{array}$ & $\begin{array}{l}\text { Auf der Erdoberfläche eintreffende kurzwellige Solarstrahlung (direkt und diffus) abzüglich der re- } \\
\text { flektierten Strahlung (bestimmt durch Albedo), positives Vorzeichen indiziert mehr Einstrahlung als } \\
\text { Abstrahlung }\end{array}$ & $W^{-2}$ \\
\hline surf_net_therm_rad & $\begin{array}{l}\text { Max. Wert, Arith. } \\
\text { Mittel }\end{array}$ & $\begin{array}{l}\text { Netto-Wärmestrahlung an der Erdoberfläche, positives Vorzeichen indiziert mehr Abstrahlung als Ein- } \\
\text { strahlung }\end{array}$ & $\mathrm{W} \mathrm{m}^{-2}$ \\
\hline surf_press & Arith. Mittel & Luftdruck & $\mathrm{Pa}$ \\
\hline total_et & Summe & Gesamte Evapotranspiration, positive Werte zeigen Evapotranspiration an, negative Werte Kondensation & $\mathrm{mm}$ \\
\hline prec & Summe & Gesamte Niederschlagsmenge (flüssig und gefroren) & $\mathrm{mm}$ \\
\hline volsw_123 & Arith. Mittel & Wasseranteil in der obersten Bodenschicht (0 bis $100 \mathrm{~cm}$ Tiefe) & $\mathrm{m}^{3} \mathrm{~m}^{-3}$ \\
\hline volsw_4 & Arith. Mittel & Wasseranteil in der unteren Bodenschicht (100 bis $289 \mathrm{~cm}$ Tiefe) & $\mathrm{m}^{3} \mathrm{~m}^{-3}$ \\
\hline
\end{tabular}

Tab. 5 Topographische Attribute

\begin{tabular}{|c|c|c|c|}
\hline Attribut & Beschreibung & Einheit & Datengrundlage \\
\hline area_calc & Aggregierte topographische Einzugsgebietsfläche & $\mathrm{km}^{2}$ & $\begin{array}{l}\text { HAO (2007), HydroATLAS (Linke et al. } \\
\text { 2019) }\end{array}$ \\
\hline elev_mean & Mittlere Einzugsgebietshöhe & $\mathrm{m} \mathrm{Mh}$ & $\begin{array}{l}\text { NASA JPL SRTMGL1 V3 Digital Elevation } \\
30 \mathrm{~m} \text { (Farr et al. 2007) }\end{array}$ \\
\hline elev_med & Mediane Einzugsgebietshöhe & $\mathrm{m} \mathrm{Mh}$ & Siehe oberhalb \\
\hline elev_std & Standardabweichung der Einzugsgebietshöhen & $\mathrm{m} \mathrm{Mh}$ & Siehe oberhalb \\
\hline elev_ran & Spannweite der Einzugsgebietshöhen (max. - min. Höhenwert) & $\mathrm{m} \mathrm{Mh}$ & Siehe oberhalb \\
\hline slope_mean & Mittlere Einzugsgebietsneigung; Horn (1981) & $\mathrm{m} \mathrm{km}^{-1}$ & Siehe oberhalb \\
\hline mvert_dist & $\begin{array}{l}\text { Horizontaler Abstand vom entferntesten Punkt des Einzugsgebietsrandes zum zugehöri- } \\
\text { gen Pegel (Länge des EZG) }\end{array}$ & $\mathrm{km}$ & $\begin{array}{l}\text { HAO (2007), HydroATLAS (Linke et al. } \\
\text { 2019) }\end{array}$ \\
\hline mvert_ang & $\begin{array}{l}\text { Winkel zwischen Nordrichtung und Verbindung vom entferntesten Punkt des Einzugs- } \\
\text { gebiets zum zugehörigen Pegel, z. B. Richtung von Norden (entferntester Punkt) nach } \\
\text { Süden (Pegel): } 180^{\circ} \text {, Richtung von Osten nach Westen: } 270^{\circ}\end{array}$ & Grad & $\begin{array}{l}\text { HAO (2007), HydroATLAS (Linke et al. } \\
\text { 2019) }\end{array}$ \\
\hline elon_ratio & $\begin{array}{l}\text { Längenstreckung } R_{\mathrm{e}} \text { nach Schumm (1956), Verhältnis des Durchmessers } D \text { eines äquiva- } \\
\text { lenten Kreises mit der Fläche } \\
\text { des Einzugsgebiets zur Länge } L \text { (mvert_dist), } R_{\mathrm{e}}=\frac{1}{L} \times \sqrt{\frac{4 \times A}{\pi}}=\frac{D}{L}\end{array}$ & - & $\begin{array}{l}\text { HAO (2007), HydroATLAS (Linke et al. } \\
\text { 2019) }\end{array}$ \\
\hline strm_dens & $\begin{array}{l}\text { Flussdichte } D_{F} \text {, Verhältnis der Flusslängen } L_{F} \text { zur Einzugsgebietsfläche A (area_calc) } \\
D_{F}=\frac{\sum L_{F}}{A}\end{array}$ & $\mathrm{~km} \mathrm{~km}^{-2}$ & $\begin{array}{l}\text { EU-Hydro - River Network Database } \\
\text { (EEA 2019) }\end{array}$ \\
\hline
\end{tabular}




\begin{tabular}{|c|c|c|c|}
\hline Attribut & Beschreibung & Einheit & Datengrundlage \\
\hline p_mean & Mittlere tägl. Niederschlagssumme & $\mathrm{mm} \mathrm{d}^{-1}$ & ERA5L (Muñoz Sabater 2019a) ${ }^{\text {a }}$ \\
\hline et0_mean & Mittlere tägl. Referenz-Evapotranspiration ET0 & $\mathrm{mm} \mathrm{d}^{-1}$ & $\begin{array}{l}\text { Global Aridity Index and Potenzial } \\
\text { Evapotranspiration (ETO) Climate } \\
\text { Database v2 (Trabucco und Zomer } \\
\text { 2019) }\end{array}$ \\
\hline eta_mean & Mittlere tägl. aktuelle Evapotranspiration & $\mathrm{mm} \mathrm{d}^{-1}$ & ERA5L (Muñoz Sabater 2019a) a \\
\hline arid_1 & $\begin{array}{l}\text { Aridität, Verhältnis zwischen mittlerer tägl. ET0 (Trabucco und Zomer 2019) und Nieder- } \\
\text { schlag (Muñoz Sabater 2019a) }\end{array}$ & - & $\begin{array}{l}\text { ERA5L (Muñoz Sabater 2019a) }{ }^{\text {a }} \text {, } \\
\text { Global Aridity Index and Potenzial } \\
\text { Evapotranspiration (ETO) Climate } \\
\text { Database v2 (Trabucco und Zomer } \\
\text { 2019) }\end{array}$ \\
\hline p_season & $\begin{array}{l}\text { Saisonalität und Zeitpunkt des Niederschlags (abgeschätzt mit Hilfe von Sinuskurven) zur } \\
\text { Darstellung der jährlichen Niederschlagszyklen, positive (negative) Werte zeigen an, dass } \\
\text { die Niederschlagssummen während der Sommer- (Winter-) Monate höher sind, Werte } \\
\text { nahe } 0 \text { indizieren gleichmäßige Niederschlagsverteilung über das Jahr; Gl. (14) in Woods } \\
\text { (2009) }\end{array}$ & - & Siehe oberhalb \\
\hline frac_snow & $\begin{array}{l}\text { Anteil des Niederschlags, der als Schnee fällt (Niederschlag an Tagen mit einer negativen } \\
\text { mittleren Tagestemperatur) }\end{array}$ & - & Siehe oberhalb \\
\hline hi_prec_fr & Häufigkeit der Starkniederschlagstage ( $\geq 5$-fache mittlere tägl. Niederschlagssumme) & Tage $\mathrm{yr}^{-1}$ & Siehe oberhalb \\
\hline hi_prec_du & $\begin{array}{l}\text { Mittlere Dauer der Starkniederschlagsperioden (Anzahl der zusammenhängenden Tage mit } \\
\geq 5 \text {-facher mittlerer tägl. Niederschlagssumme) }\end{array}$ & Tage & Siehe oberhalb \\
\hline hi_prec_ti & $\begin{array}{l}\text { Saison, in der die meisten niederschlagsreichen Tage ( } \geq 5 \text {-fache mittlere tägl. Nieder- } \\
\text { schlagssumme) auftreten }\end{array}$ & Saison ${ }^{\mathrm{c}}$ & Siehe oberhalb \\
\hline lo_prec_fr & Häufigkeit der Trockentage ( $<1 \mathrm{~mm} \mathrm{~d}^{-1}$ Niederschlag) & Tage $\mathrm{yr}^{-1}$ & Siehe oberhalb \\
\hline lo_prec_du & $\begin{array}{l}\text { Mittlere Dauer der Trockenphasen (Anzahl der zusammenhängenden Tage }<1 \mathrm{~mm} \mathrm{~d}^{-1} \\
\text { Niederschlag) }\end{array}$ & Tage & Siehe oberhalb \\
\hline lo_prec_ti & Saison, in der die meisten niederschlagsarmen Tage $\left(<1 \mathrm{~mm} \mathrm{~d}^{-1}\right.$ Niederschlag) auftreten & Saison ${ }^{c}$ & Siehe oberhalb \\
\hline
\end{tabular}

Tab. 7 Hydrologische Attribute

\begin{tabular}{|c|c|c|c|}
\hline Attribut & Beschreibung & Einheit & Datengrundlage \\
\hline q_mean & Mittlere tägl. Abflusshöhe & $\mathrm{mm} \mathrm{d}^{-1}$ & $\begin{array}{l}\text { BAFU (2020), CHMI (2020), } \\
\text { GKD (2020), HZB (2020), } \\
\text { LUBW (2020) }^{\text {a }}\end{array}$ \\
\hline runoff_ratio & Abflusskoeffizient, Quotient zwischen mittlerem tägl. Abfluss und Niederschlag & - & $\begin{array}{l}\text { BAFU (2020), CHMI (2020), } \\
\text { GKD (2020), HZB (2020), } \\
\text { LUBW (2020) }^{\text {a }} \text {, ERA5L } \\
{\text { (Muñoz Sabater } 2019 a)^{a}}^{\text {a }}\end{array}$ \\
\hline stream_elas & $\begin{array}{l}\text { Abfluss-Niederschlags-Elastizität, d. h. Sensitivität des Abflusses gegenüber Änderun- } \\
\text { gen des Niederschlags auf jährlicher Zeitskala unter Verwendung des mittlerern tägl. } \\
\text { Abflusses als Referenz; Gl. (7) in Sankarasubramanian et al. (2001), wobei das letzte } \\
\text { Element } \bar{P} / \bar{Q} \text { und nicht } \bar{Q} / \bar{P} \text { ist }\end{array}$ & - & $\begin{array}{l}\text { BAFU (2020), CHMI (2020), } \\
\text { GKD (2020), HZB (2020), } \\
\text { LUBW (2020) }^{\text {a }}\end{array}$ \\
\hline slope_fdc & $\begin{array}{l}\text { Steigung der Dauerlinie zwischen log-transformierten } \\
\text { 33. and 66. Abflussperzentil; Gl. (3) in Sawicz et al. (2011) }\end{array}$ & - & Siehe oberhalb \\
\hline baseflow_index_ladson & $\begin{array}{l}\text { Basisabfluss-Index, Quotient zwischen mittlerem tägl. Basisabfluss sowie Abfluss, } \\
\text { Ganglinienseparation wurde mit dem Ladson et al. (2013) Digitalfilter durchgeführt }\end{array}$ & - & Siehe oberhalb \\
\hline baseflow_index_lfstat & $\begin{array}{l}\text { Basisabfluss-Index, Quotient zwischen mittlerem tägl. Basisabfluss sowie Abfluss, } \\
\text { Ganglinienseparation wurde mit dem Package von Tallaksen und Van Lanen (2004) } \\
\text { durchgeführt }\end{array}$ & - & Siehe oberhalb \\
\hline hfd_mean & $\begin{array}{l}\text { Zeitpunkt der halben Jahresabflussfracht, d. h. Anzahl der Tage seit dem Beginn } \\
\text { des hydrologischen Jahres (01. Oktober) bis die mittlere halbe Jahresabflussfracht } \\
\text { erreicht wird; Court (1962) }\end{array}$ & $\begin{array}{l}\text { Tage seit } \\
\text { 01. Oktober }\end{array}$ & Siehe oberhalb \\
\hline Q5 & 5. Abflussperzentil (Niederwasser) & $\mathrm{mm} \mathrm{d}^{-1}$ & Siehe oberhalb \\
\hline Q95 & 95. Abflussperzentil (Hochwasser) & $\mathrm{mm} \mathrm{d}^{-1}$ & Siehe oberhalb \\
\hline high_q_freq & $\begin{array}{l}\text { Häufigkeit der Tage mit sehr hohem Abfluss (>9-facher medianer Tagesabfluss); } \\
\text { Clausen und Biggs (2000), Tab. } 2 \text { in Westerberg und McMillan (2015) }\end{array}$ & Tage $\mathrm{yr}^{-1}$ & Siehe oberhalb \\
\hline high_q_dur & $\begin{array}{l}\text { Dauer der Phasen mit sehr hohem Abfluss (Anzahl der zusammenhängenden Tage } \\
\text { mit > 9-fachem medianen Tagesabfluss); Clausen und Biggs (2000), Tab. } 2 \text { in Wester- } \\
\text { berg und McMillan (2015) }\end{array}$ & Tage & Siehe oberhalb \\
\hline
\end{tabular}




\begin{tabular}{|c|c|c|c|}
\hline Attribut & Beschreibung & Einheit & Datengrundlage \\
\hline low_q_freq & $\begin{array}{l}\text { Häufigkeit der Tage mit sehr geringem Abfluss ( }<0,2-\text { facher mittlerer Tagesabfluss); } \\
\text { Olden und Poff (2003), Tab. } 2 \text { in Westerberg und McMillan (2015) }\end{array}$ & Tage $\mathrm{yr}^{-1}$ & Siehe oberhalb \\
\hline low_q_dur & $\begin{array}{l}\text { Dauer der Phasen mit sehr geringem Abfluss (Anzahl der zusammenhängenden } \\
\text { Tage mit }<0,2 \text {-fachem mittleren Tagesabfluss); Olden und Poff (2003), Tab. } 2 \text { in } \\
\text { Westerberg und McMillan (2015) }\end{array}$ & Tage & Siehe oberhalb \\
\hline zero_q_freq & Anteil der Tage ohne Abfluss & $\%$ & Siehe oberhalb \\
\hline
\end{tabular}

Tab. 8 Attribute zur Landbedeckung

\begin{tabular}{|c|c|c|c|}
\hline Attribut & Beschreibung & Einheit & Datengrundlage \\
\hline IC_dom & 3-Ziffern Code für die vorherrschende Landnutzungsklasse (CLC - Corine Land Cover) ${ }^{\text {a }}$ & - & CORINE (2012) \\
\hline agr_fra & Anteil der landwirtschaftlich genutzten Fläche (alle CLC Klassen, welche mit der Ziffer 2 beginnen) ${ }^{a}$ & - & Siehe oberhalb \\
\hline bare_fra & Anteil an kahler Fläche (CLC Klassen 332, 333) ${ }^{a}$ & - & Siehe oberhalb \\
\hline forest_fra & Anteil an Wald (CLC Klassen $311,312,313)^{a}$ & - & Siehe oberhalb \\
\hline glac_fra & Anteil an Gletscher (CLC Klasse 335) ${ }^{\text {a }}$ & - & Siehe oberhalb \\
\hline lake_fra & Anteil an natürlichen oder künstlichen Wasserkörpern mit ganzjähriger Wasserfüllung (CLC Klasse 512) a & - & Siehe oberhalb \\
\hline urban_fra & $\begin{array}{l}\text { Anteil der hauptsächlich von Gebäuden belegten Flächen einschließlich der Infrastruktur (CLC Klassen 111, } \\
112,121,122,123,124)^{\text {a }}\end{array}$ & - & Siehe oberhalb \\
\hline
\end{tabular}

Tab. 9 Attribute zur Vegetation

\begin{tabular}{|c|c|c|c|}
\hline Attribut & Beschreibung & Einheit & Datengrundlage \\
\hline lai_max & $\begin{array}{l}\text { Maximaler Monatsmittelwert des einseitigen Blattlächenindex (basierend auf } \\
12 \text { monatlichen Mitteln) }\end{array}$ & - & M0DIS MCD15A3H (Myneni et al. 2015) \\
\hline lai_diff & $\begin{array}{l}\text { Differenz zwischen maximalem und minimalem Monatsmittelwert des einseiti- } \\
\text { gen Blatttlächenindex (basierend auf } 12 \text { monatlichen Mitteln) }\end{array}$ & - & Siehe oberhalb \\
\hline ndvi_max & Maximaler Monatsmittelwert des NDVI (basierend auf 12 monatlichen Mitteln) & - & M0DIS M0D09Q1 (Vermote 2015) \\
\hline ndvi_min & Minimaler Monatsmittelwert des NDVI (basierend auf 12 monatlichen Mitteln) & - & Siehe oberhalb \\
\hline gvf_max & $\begin{array}{l}\text { Maximaler Monatsmittelwert der GVF (green vegetation fraction) (basierend auf } \\
12 \text { monatlichen Mitteln) }\end{array}$ & - & $\begin{array}{l}\text { MODIS MOD09Q1 (Vermote 2015), MODIS } \\
\text { MCD12Q1 (Friedl und Sulla-Menashe 2019) }\end{array}$ \\
\hline gvf_diff & $\begin{array}{l}\text { Differenz zwischen maximalem und minimalem Monatsmittelwert der GVF } \\
\text { (green vegetation fraction) (basierend auf } 12 \text { monatlichen Mitteln) }\end{array}$ & - & Siehe oberhalb \\
\hline
\end{tabular}

Tab. 10 Bodenattribute

\begin{tabular}{|c|c|c|c|}
\hline Attribut & Beschreibung & Einheit & Datengrundlage \\
\hline bedrk_dep & Tiefe zum Grundgestein, max. Tiefe des Ausgangsdatensatzes ist $50 \mathrm{~m}$ & $\mathrm{~m}$ & Pelletier et al. (2016) \\
\hline root_dep & Verfügbare Wurzeltiefe, max. Tiefe des Ausgangsdatensatzes ist $1,5 \mathrm{~m}^{\text {a }}$ & $\mathrm{m}$ & $\begin{array}{l}\text { European Soil Database Derived data (Hiederer } \\
\text { 2013a, b) }\end{array}$ \\
\hline soil_poros & Bodenporosität ${ }^{a, b}$ & - & Siehe oberhalb \\
\hline soil_condu & $\begin{array}{l}\text { Gesättigte hydraulische Leitfähigkeit, max. Tiefe des Ausgangsdatensatzes } \\
\text { ist } 2 \mathrm{~m}^{\mathrm{a}, \mathrm{b}}\end{array}$ & $\mathrm{Cm} \mathrm{hr}^{-1}$ & 3D Soil Hydraulic Database of Europe (Toth et al. 2017) \\
\hline soil_tawc & $\begin{array}{l}\text { Verfügbare Bodenwasserkapazität TAWC (Anteil zwischen Feldkapazität } \\
\text { und permanentem Welkepunkt) }{ }^{\mathrm{a}, \mathrm{b}}\end{array}$ & $\mathrm{m}$ & $\begin{array}{l}\text { European Soil Database Derived data (Hiederer } \\
\text { 2013a, b) }\end{array}$ \\
\hline sand_fra & Sandanteil (vom Bodenmaterial $<2 \mathrm{~mm}$ ) a,b & - & Siehe oberhalb \\
\hline silt_fra & Schluffanteil (vom Bodenmaterial $<2 \mathrm{~mm}$ ) a,b & - & Siehe oberhalb \\
\hline clay_fra & Tonanteil (vom Bodenmaterial $<2 \mathrm{~mm})^{\mathrm{a}, \mathrm{b}}$ & - & Siehe oberhalb \\
\hline grav_fra & Anteil an Kies ${ }^{a, b}$ & - & Siehe oberhalb \\
\hline oc_fra & Anteil an organischem Material ${ }^{a, b}$ & - & Siehe oberhalb \\
\hline
\end{tabular}




\begin{tabular}{|c|c|c|c|}
\hline Attribut & Beschreibung & Einheit & Datengrundlage \\
\hline gc_dom & Vorherrschende geologische Klasse & - & GLiM (Hartmann und Moosdorf 2012) \\
\hline gc_ig_fra & Anteil der Klasse „Eis und Gletscher“ (ig) & - & Siehe oberhalb \\
\hline gc_mt_fra & Anteil „Metamorphite“ (mt) & - & Siehe oberhalb \\
\hline gc_pa_fra & Anteil „saure Plutonite“ (pa) & - & Siehe oberhalb \\
\hline gc_pb_fra & Anteil „,basische Plutonite“ (pb) & - & Siehe oberhalb \\
\hline gc_pi_fra & Anteil „intermediäre Plutonite“ (pi) & - & Siehe oberhalb \\
\hline gc_py_fra & Anteil „Pyroklastika“ (py) & - & Siehe oberhalb \\
\hline gc_sc_fra & Anteil „karbonatisches Sedimentgestein“ (sc) & - & Siehe oberhalb \\
\hline gc_sm_fra & Anteil „gemischtes Sedimentgestein“ (sm) & - & Siehe oberhalb \\
\hline gc_ss_fra & Anteil „siliklastisches Sedimentgestein“(ss) & - & Siehe oberhalb \\
\hline gc_su_fra & Anteil „unverfestigte Sedimente“ (su) & - & Siehe oberhalb \\
\hline gc_va_fra & Anteil „saure Vulkanite“ (va) & - & Siehe oberhalb \\
\hline gc_vb_fra & Anteil „basische Vulkanite“ (vb) & - & Siehe oberhalb \\
\hline gc_wb_fra & Anteil „Wasserkörper“ (wb) & - & Siehe oberhalb \\
\hline geol_perme & Untergrund-Permeabilität (auf Basis log10), Berücksichtigung von Permafrost & - & GLHYMPS (Gleeson et al. 2014) \\
\hline geol_poros & Untergrund-Porosität & - & Siehe oberhalb \\
\hline
\end{tabular}

\section{Literatur}

Addor, N., Do, H. X., Alvarez-Garreton, C., Coxon, G., Fowler, K., Mendoza, P. A. (2019): Largesample hydrology: recent progress, guidelines for new datasets and grand challenges, Hydrolog. Sci. J., 65, S. 712-725, https://doi.org/10 1080/02626667.2019.1683182

Addor, N., Newman, A. J., Mizukami, N., Clark, M. (2017a): The CAMELS data set: catchmen attributes and meteorology for large-sample studies, Hydrol. Earth Syst. Sci., 21, S. 5293-5313, https://doi.org/10.5194/hess-21-5293-2017 Addor, N. (2017b): R-Skripts für die Reproduktion der klimatischen und hydrologischen Indizes, sowie zur Erstellung der Karten, GitHub, verfügbar unter: https://github.com/naddor/camels (letzter Zugriff: 02.03.2020)

Allen, R. G., Pereira, L. S., Raes, D., Smith, M. (1998): Crop Evapotranspiration. Guidelines for Computing Crop Water Requirements, FAO Irrigation and Drainage Paper 56, Food and Agriculture Organization (FAO) of the United Nations, Rom, ISBN 92-5-104219-5, 300pp

Alvarez-Garreton, C., Mendoza, P. A., Boisier, J. P., Addor, N., Galleguillos, M., ZambranoBigiarini, M., Lara, A., Puelma, C., Cortes, G. Garreaud, R., McPhee, J., Ayala, A. (2018): The CAMELS-CL dataset: catchment attributes and meteorology for large sample studies-Chile dataset, Hydrol. Earth Syst. Sci., 22, S. 5817-5846, https://doi.org/10.5194/hess-22-5817-2018 Arora, V. K. (2002): The use of the aridity index to assess climate change effect on annual runoff, Journal of Hydrol., 265, S. 164-177, https://doi. org/10.1016/S0022-1694(02)00101-4 BAFU (2020): Bundesamt für Umwelt - Abteilung Hydrologie, Bern, Schweiz (erhalten am: 23.09.2020)

Barnes, W. L., Xiong, X., Salomonson, V. V. (2003): Status of terra MODIS and aqua modis, Adv. in Space Res., 32(11), S. 2099-2106 https://doi.org/10.1016/S0273-1177(03)90529-1

Beck, H. E., Van Dijk, A. I. J. M., Miralles, D. G., De Jeu, R. A. M., Bruijnzeel, L. A., McVicar, T. R. Schellekens, J. (2013): Global patterns in base flow index and recession based on streamflow observations from 3394 catchments, Water Resour. Res., 49, S. 7843-7863, https://doi.org/10 1002/2013WR013918
Beck, H. E., Vergopolan, N., Pan, M., Levizzani, V., van Dijk, A. I. J. M., Weedon, G. P., Brocca, L., Pappenberger, F., Huffman, G. J., Wood, E. F. (2017): Global-scale evaluation of 22 precipitation datasets using gauge observations and hydrological modeling, Hydrol. Earth Syst. Sci. 21, S. 6201-6217, https://doi.org/10.5194/hess21-6201-2017

Beck, H. E., Wood, E. F., Pan, M., Fisher, C. K., Miralles, D. G., van Dijk, A. I. J. M., McVicar, T R., Adler, R. F. (2019): MSWEP V2 Global 3-Hourly 0.1 Precipitation: Methodology and Quantitative Assessment, B. Am. Meteorol. Soc., 100(3), S. 473-500, https://doi.org/10.1175/BAMS-D-170138.1

Berghuijs, W. R., Sivapalan, M., Woods, R. A. Savenije, H. H. G. (2014): Patterns of similarity of seasonal water balances: A window into streamflow variability over a range of time scales, Water Resour. Res., 50, S. 5638-5661, https://doi. org/10.1002/2014WR015692

Blöschl, G., Hall, J., Viglione, A., Perdigão, R. A P., Parajka, J., et al. (2019a): Changing climate both increases and decreases European rive floods, Nature, 573, S. 108-111, https://doi.org/ 10.1038/s41586-019-1495-6

Blöschl, G., Bierkens, M. F. P., Chambel, A. Cudennec, C., Destouni, G., et al. (2019b): Twenty-three unsolved problems in hydrology (UPH) - a community perspective, Hydrolog Sci. J., 64, S. 1141-1158, https://doi.org/10.1080/ 02626667.2019.1620507

Blöschl, G., Sivapalan, M., Savenije, H., Wagener, T., Viglione, A. (Eds.) (2013): Runoff prediction in ungauged basins: synthesis across processes, places and scales, Cambridge University Press, Cambridge, ISBN 9781107028180, 490pp BMLFUW (2013): Hydrographisches Jahrbuch von Österreich 2013, Bundesministerium für Landwirtschaft, Regionen und Tourismus - Hydrographisches Zentralbüro, Wien, Österreich, verfügbar unter: https://www.bmlrt.gv.at/dam/ jcr:bb100102-eac3-4b51-8d8d-f40b94e5d546/

Jahrbuch_2013_Datenteil.pdf (letzter Zugriff: 16.12.2020)

Boer-Euser, T, de, McMillan, H. K, Hrachowitz, M. Winsemius, H. C., Savenije, H. H. G. (2016) Influence of soil and climate on root zone stora- ge capacity, Water Resour. Res., 52, S. 2009-2024, https://doi.org/10.1002/2015WR018115 Bossard, M., Feranec, J., Otahel, J. (2000): CORINE land cover technical guide-Addendum 2000, Technical report No 40, European Environment Agency, Kopenhagen, Dänemark, 105pp Broxton, P. D., Zeng, X., Scheftic, W., Troch P. A. (2014): A MODIS-Based Global 1-km Maximum Green Vegetation Fraction Dataset, Journal of applied Meteorology and Climatology, 53, S. 1996-2004, https://doi.org/10.1175/JAMC-D13-0356.1

Budyko, M. I. (1974): Climate and Life; Academic Press: New York, NY, USA

Büttner, G., Maucha, G. (2006): The thematic accuracy of Corine land cover 2000-Assessmen using LUCAS (land use/cover area frame statistical survey), Technical report No 7/2006, European Environment Agency, Kopenhagen, Dänemark, ISBN 92-9167-844-9, 90pp

Chagas, V. B. P., Chaffe, P. L. B., Addor, N., Fan, F M., Fleischmann A. S., Paiva, R. C. D, Siqueira V. A. (2020): CAMELS-BR: hydrometeorological time series and landscape attributes for 897 catchments in Brazil, Earth Syst. Sci. Data, 12, S. 2075-2096, https://doi.org/10.5194/essd-122075-2020

Chapman, T. (1999): A comparison of algorithms for stream flow recession and baseflow separation, Hydrol. Processes, 13(5), S. 701-714. https://doi.org/10.1002/(SICI) 1099- 1085(19990 415) 13:5<701::AID-HYP774>3.0.CO;2-2

CHMI (2020): Tschechisches Hydrometeorologisches Institut, Brünn, Tschechische Republik (erhalten am: 14.12.2020)

Clausen, B., Biggs, B. J. F. (2000): Flow variables for ecological studies in temperate streams: groupings based on covariance, J. Hydrol., 237, S. 184-197, https://doi.org/10.1016/S00221694(00)00306-1

COPa (2021): Europäische Weltraumorganisation \& Europäische Kommission, Copernicus Programm, Copernicus Open Access Hub, verfügbar unter: https://scihub.copernicus.eu/ (letzter Zugriff: 22.02.2021)

COPb (2021): Europäische Weltraumorganisation \& Europäische Kommission, Copernicus Programm, Copernicus Climate Data Store, verfüg- 
bar unter: https://cds.climate.copernicus.eu/\#!/ home (letzter Zugriff: 22.02.2021) CORINE (2012): CORINE Land Cover 2012, Europäische Umweltagentur, Kopenhagen, Dänemark, verfügbar unter: https://land.copernicus. eu/pan-european/corine-land-cover (letzter Zugriff: 02.03.2020)

Court, A. (1962): Measures of streamflow timing, J. Geophys. Res., 67, S. 4335-4339, https://doi. org/10.1029/JZ067i011p04335

Coxon, G., Addor, N., Bloomfield, J. P., Freer, J., Fry, M., Hannaford, J., Howden, N. J. K., Lane, R., Lewis, M., Robinson, E. L., Wagener, T. Woods, R. (2020): CAMELS-GB: Hydrometeorological time series and landscape attributes for 671 catchments in Great Britain, Earth Syst. Sci Data, 12, S. 2459-2483, https://doi.org/10.5194/ essd-12-2459-2020

Do, H. X., Gudmundsson, L., Leonard, $M$., Westra, S. (2018): The Global Streamflow Indices and Metadata Archive (GSIM) —Part 1: The production of a daily streamflow archive and metadata, Earth Syst. Sci. Data, 10, S. 765-785 https://doi.org/10.5194/essd-10-765-2018

Döll, P., Douville, H., Güntner, A., Schmied, $H$. M., Wada, Y. (2016): Modelling freshwater resources at the global scale: challenges and prospects, Surv. Geophys., 37, S. 195-221, https:// doi.org/10.1007/s10712-015-9343-1

Duan, Q., Schaake, J., Andréassian, V., Franks, S., Goteti, G., Gupta, H. V., Gusev, Y. M., Habets, F., Hall, A., Hay, L., Hogue, T., Huang, M., Leavesley, G., Liang, X., Nasonova, O. N., Noilhan, J., Oudin, L., Sorooshian, S., Wagener, T., Wood, E. F. (2006): Model Parameter Estimation Experiment (MOPEX): An overview of science strategy and major results from the second and third workshops, Journal of Hydrology, 320, S. 3-17, https://doi.org/10.1016/j.jhydrol.2005.07.031

Eckhardt, K. (2008): A comparison of baseflow indices, which were calculated with seven different baseflow separation methods, J. of Hydrol., 352, S. 168-173, https://doi.org/10.1016/j. jhydrol.2008.01.005

EEA (2019): EU-Hydro - River Network Database, Version 1.2, Europäische Umweltagentur Copernicus Programm, verfügbar unter: https:// land.copernicus.eu/imagery-in-situ/eu-hydro/ eu-hydro-river-network-database (letzter $\mathrm{Zu}$ griff: 22.10 .2020$)$

ESA (2021): Europäische Weltraumorganisation, Copernicus Programm, Sentinel Mission, Sentinel Online, verfügbar unter: https://sentinels. copernicus.eu/web/sentinel/home (letzter $\mathrm{Zu}$ griff: 22.02.2021)

ESDB (2004): The European Soil Database distribution version 2.0, European Commission and the European Soil Bureau Network, CDROM, EUR 19945 EN

Falkenmark, M., Chapman, T. (1989): Comparative hydrology: An ecological approach to land and water resources, UNESCO, Paris

Fan, Y. (2015): Groundwater in the Earth's critical zone: Relevance to large-scale patterns and processes: Groundwater at large scales, Wate Resour. Res., 51, S. 3052-3069, https://doi.org/ 10.1002/2015WR017037

Fan, Y., Clark, M., Lawrence, D. M., Swenson, S., Band, L. E., et al. (2019): Hillslope Hydrology in Global Change Research and Earth System Modeling, Water Resour. Res., 55, S. 1737-1772, https://doi.org/10.1029/2018WR023903

Farr, T. G., Rosen, P. A., Caro, E., Crippen, R. Duren, R., Hensley, S., Kobrick, M., Paller, M. Rodriguez, E., Roth, L., Seal, D., Shaffer, S., Shimada, J., Umland, J., Werner, M., Oskin, M. Burbank, D., Alsdorf, D. E. (2007): The shuttle radar topography mission: Reviews of Geophysics, 45(2), RG2004, https://doi.org/10.1029/ 2005RG000183

Friedl, M., Sulla-Menashe, D. (2019): MCD12Q MODIS/Terra+Aqua Land Cover Type Yearly L3
Global 500m SIN Grid V006 [Data set], NASA EOSDIS Land Processes DAAC, verfügbar unter https://doi.org/10.5067/MODIS/MCD12Q1.006 (letzter Zugriff: 12.03.2020)

Funk, C., Peterson, P., Landsfeld, M., Pedreros, D., Verdin, J., Shukla, S., Husak, G., Rowland, J., Harrison, L., Hoell, A., Michaelsen, J. (2015): The climate hazards infrared precipitation with stations-a new environmental record for monitoring extremes, Scientific Data, 2, Article Number: 150066 (2015), https://doi.org/10. 1038/sdata.2015.66

GEEa (2021); Google Earth Engine Platform, verfügbar unter: https://earthengine.google. com/platform/ (letzter Zugriff: 22.02.2021) GEEb (2021): Google Earth Engine Datenkatalog, verfügbar unter: https://developers.google. com/earth-engine/datasets (letzter Zugriff 22.01.2021)

Ghiggi, G., Humphrey, V., Seneviratne, S. I., Gudmundsson, L. (2019): GRUN: an observation-based global gridded runoff dataset from 1902to 2014, Earth Syst. Sci. Data, 11, S 1655-1674, https://doi.org/10.5194/essd-11$1655-2019$

GKD (2020): Bayerisches Landesamt für Umwelt - Gewässerkundlicher Dienst, München, Deutschland, verfügbar unter: https://www.gkd. bayern.de/de/fluesse/abfluss/tabellen (heruntergeladen am: 15.09.2020)

Gleeson, T., Moosdorf, N., Hartmann, J., van Beek, L. P. H. (2014): A glimpse beneath earth's surface: GLobal HYdrogeology MaPS (GLHYMPS) of permeability and porosity, Geophys. Res. Lett., 41, S. 3891-3898, https://doi.org/10.1002/ 2014GL059856, verfügbar unter: https:// dataverse.scholarsportal.info/dataset.xhtml? persistentId=doi:10.5683/SP2/DLGXYO (letzter Zugriff: 12.03.2020)

Gorelick, N., Hancher, M., Dixon, M., Ilyushchenko, S., Thau, D., Moore, R. (2017): Google Earth Engine: Planetary-scale geospatial analysis for everyone, Remote Sensing of Environment, 202, S. 18-27, https://doi.org/10.1016/j.rse.2017. 06.031

Gudmundsson, L., Do, H. X., Leonard, M. Westra, S. (2018): The Global Streamflow Indices and Metadata Archive (GSIM) - Part 2: Quality control, time-series indices and homogeneity assessment, Earth Syst. Sci. Data, 10, S 787-804, https://doi.org/10.5194/essd-10-7872018

Gudmundsson, L., Leonard, M., Do, H. X., Westra, S., and Seneviratne, S. I. (2019): Observed Trends in Global Indicators of Mean and Extreme Streamflow, Geophys. Res. Lett., 46 S. 756-766, https://doi.org/10.1029/ 2018GL079725

Gupta, H. V., Perrin, C., Blöschl, G., Montanari, A., Kumar, R., Clark, M., Andréassian, V. (2014): Large-sample hydrology: a need to balance depth with breadth, Hydrol. Earth Syst. Sci., 18, S. 463-477, https://doi.org/10.5194/ hess-18-463-2014

HAO (2007): Hydrologischer Atlas Österreichs (digHAO), 3. Ausgabe, Bundesministerium fü Landwirtschaft, Regionen und Tourismus - Hydrographisches Zentralbüro, Wien, Österreich Hargreaves, G. H. (1994): Defining and Using Reference Evapotranspiration, Journal of Irrigation and Drainage Engineering, 120(6), S. 1132-1139, https://doi.org/10.1061/(ASCE) 0733-9437(1994)120:6(1132)

Hartmann, J., Moosdorf, N. (2012): The new global lithological map database GLiM: A representation of rock properties at the Earth surface, Geochem. Geophy. Geosy., 13, S. 1-37, https:// doi.org/10.1029/2012GC004370

Hengl, T., Mendes de Jesus, J., Heuvelink, G. B. M., Ruiperez Gonzalez, M., Kilibarda, M. Blagoti'c, A., Shangguan, W., Wright, M. N. Geng, X., Bauer-Marschallinger, B., Guevara,
M.A, Vargas, R, MacMillan, R. A, Batjes, N. H., Leenaars, J. G. B., Ribeiro, E., Wheeler, I. Mantel, S., Kempen, B. (2017): SoilGrids250m: Global gridded soil information based on machine learning, edited by: Bond-Lamberty, B. PLoS ONE, 12, e0169748, https://doi.org/10 1371/journal.pone.0169748

Hennermann, K., Guillory, A. (2020): ERA5 uncertainty estimation, CDS dataset documentation, European Centre for Medium-Range Weather Forecasts (ECMWF), verfügbar unter: https://confluence.ecmwf.int/display/CKB/ ERA5\%3A+uncertainty+estimation (letzter $\mathrm{Zu}$ griff: 30.11 .2020 )

Herrnegger, M., Nachtnebel, H. P., Haiden, T. (2012): Evapotranspiration in high alpine catchments-an important part of the water balance!, Hydrol. Res., 43(4), S. 460-475, https:// doi.org/10.2166/nh.2012.132

Herrnegger, M., Senoner, T., Nachtnebel, H. P. (2018): Adjustment of spatio-temporal precipitation patterns in a high Alpine environment, J. Hydrol., 556, S. 913-921, https://doi.org/10. 1016/j.jhydrol.2016.04.068

Hersbach, H., Bell, B., Berrisford, P, Hirahara, S., Horányi, A., Muñoz-Sabater, J., et al. (2020): The ERA5 global reanalysis, Quarterly Journal of the Royal Meteorol. Soc., 146(730), S. 1999-2049, https://doi.org/10.1002/qj.3803

Hiederer, R. (2013a): Mapping Soil Properties for Europe-Spatial Representation of Soil Database Attributes, Luxemburg: Publications Office of the European Union, EUR26082EN Scientific and Technical Research series, ISSN 1831-9424, https://doi.org/10.2788/94128

Hiederer, R. (2013b): Mapping Soil Typologies-Spatial Decision Support Applied to European Soil Database, Luxemburg: Publications Office of the European Union, EUR25932EN Scientific and Technical Research series, ISSN 18319424, https://doi.org/10.2788/8728

Horn, B. K. P. (1981): Hill shading and the reflectance map, Proceedings of the IEEE, 69(1), S. 14-47, https://doi.org/10.1109/PROC.1981. 11918

Hötzl, H. (1996): Origin of the Danube-Aach system, Environmental Geology, 27, S. 87-96, https://doi.org/10.1007/BF01061676

Huscroft, J., Gleeson, T., Hartmann, J., Bör ker, J. (2018): Compiling and Mapping Global Permeability of the Unconsolidated and Consolidated Earth: GLobal HYdrogeology MaPS 2.0 (GLHYMPS 2.0), Geophys. Res. Lett., 45, S. 1897-1904, https://doi.org/10.1002/ 2017GL075860

HZB (2020): Bundesministerium für Landwirtschaft, Regionen und Tourismus - Hydrographisches Zentralbüro, Wien, Österreich (erhalten am: 08.09.2020

ICPDR (2020): Danube Basin Facts \& Figures, verfügbar unter: https://www.icpdr.org/ flowpaper/viewer/default/files/nodes/

documents/icpdr facts figures.pdf (letzter $\mathrm{Zu}$ griff: 21.09 .2020$)$

Irons, J. R., Dwyer, J. L., Barsi, J. A. (2012): The next Landsat satellite: The Landsat Data Continuity Mission, Remote Sensing of Environment, 122, S. 11-21, https://doi.org/10.1016/j.rse.2011. 08.026

Klingler, C., Bernhardt, M., Wesemann, J., Schulz, K., Herrnegger, M., (2020): Lokale hydrologische Modellierung mit globalen, alternativen Datensätzen, Hydrologie und Wasserbewirtschaftung, 64(4), S. 166-187, https://doi. org/10.5675/HyWa_2020.4_1

Klingler, C., Schulz, K., Herrnegger, M. (2021): LamaH | Large-Sample Data for Hydrology and Environmental Sciences for Central Europe, Earth Syst. Sci. Data Discuss. [preprint], in review

Kobolschnig, G. R., Schöner, W. (2011): The relevance of glacier melt in the water cycle of the 
Alps: the example of Austria, Hydrol. Earth Syst Sci., 15, S. 2039-2048, https://doi.org/10.5194/ hess-15-2039-2011

Kratzert, F., Klotz, D., Brenner, C., Schulz, K. Herrnegger, M. (2018): Rainfall-runoff modelling using Long Short-Term Memory (LSTM) networks, Hydrol. Earth Syst. Sci., 22, S. 6005-6022, https://www.hydrol-earth-syst-sci.net/22/6005/ 2018

Kratzert, F., Klotz, D., Herrnegger, M., Sampson, A. K., Hochreiter, S., Nearing, G. (2019a): Toward improved predictions in ungauged basins: Exploiting the power of machine learning Water Resources Research, 55, S. 11344-11354, https://doi.org/10.1029/2019WR026065

Kratzert, F., Klotz, D., Shalev, G., Klambauer, G., Hochreiter, S., Nearing, G. (2019b): Towards learning universal, regional, and local hydrological behaviors via machine learning applied to large-sample datasets, Hydrol. Earth Syst. Sci. 23, S. 5089-5110, https://doi.org/10.5194/hess23-5089-2019

Kuentz, A., Arheimer, B., Hundecha, Y., Wagener, T. (2017): Understanding hydrologic variability across Europe through catchment classification, Hydrol. Earth Syst. Sci., 21, S. 2863-2879, https://doi.org/10.5194/hess-21-2863-2017 Kuhn, M. (2004): The Reaction of Austrian Glaciers and their Runoff to Changes in Temperature and Precipitation Levels, Österreichische Wasser- und Abfallwirtschaft, 56(1-2), S. 1-7 Ladson, A., Brown, R., Neal, B., Nathan, R. (2013): A standard approach to baseflow separation using the Lyne and Hollick filter, Australian Journal of Water Resources, 17, S. 25-34, https:// doi.org/10.7158/13241583.2013.11465417 Lambrecht, A., Kuhn, M. (2007): Glacier changes in the Austrian Alps during the last three decades, derived from the new Austrian glacier inventory, Ann. Glaciol., 46, S. 177-184, https:// doi.org/10.3189/17275640778287134 Land Tirol (2020): Katalog Gewässernetz Tirol, Landesregierung Tirol, Innsbruck, verfügbar unter: https://www.data.gv.at/katalog (heruntergeladen am: 17.10.2020)

Lehner, B., Verdin, K., Jarvis, A. (2008): New global hydrography derived from spaceborne elevation data, Eos, Transactions, AGU, 89(10), S. 93-94, https://doi.org/10.1029/2008EO100001 Linke, S., Lehner, B., Ouellet Dallaire, C., Ariwi, J., Grill, G., Anand, M., Beames, P., BurchardLevine, V., Maxwell, S., Moidu, H., Tan, F., Thieme, M. (2019): Global hydro-environmental sub-basin and river reach characteristics at high spatial resolution, Scientific Data, 6, Nr. 283 https://doi.org/10.1038/s41597-019-0300-6

LUBW (2020): Landesanstalt für Umwelt Baden-Württemberg-Gewässerkundlicher Dienst, Karlsruhe, Deutschland, verfügbar unter: http:// udo.lubw.baden-wuerttemberg.de/public/p/ pegel_messwerte_leer (erhalten am: 04.09.2020) Luke, A., Vrugt, J. A., AghaKouchak, A., Matthew, R, Sanders, B. F (2017): Predicting nonstationary flood frequencies: Evidence supports an updated stationarity thesis in the United States, Water Resour. Res., 53, S. 5469-5494, https://doi. org/10.1002/2016WR019676

Malenovsky, Z, Rott, H., Cihlar, J., Schaepman, M.E., Garcia-Santos, G., Fernandes, R., Berger, M. (2012): Sentinels for science: Potentia of Sentinel-1, -2, and -3 missions for scientific observations of ocean, cryosphere, and land, Remote Sensing of Environment, 120, S. 91-101, https://doi.org/10.1016/j.rse.2011.09.026 Muñoz Sabater, J. (2019a): ERA5-Land hourly data from 1981to present, Copernicus Climate Change Service (C3S) Climate Data Store (CDS), verfügbar unter: https://doi.org/10.24381/cds. e2161bac (letzter Zugriff: 22.10.2020)

Muñoz Sabater, J. (2019b): First ERA5-Land dataset to be released this spring, ECMWF newsletter, number 159-spring 2019, verfügbar unter: https://www.ecmwf.int/en/newsletter/ 159/news/first-era5-land-dataset-be-releasedspring (letzter Zugriff: 30.11.2020) Muñoz Sabater, J., Dutra, E., Balsamo, G., Hersbach, H., Boussetta, S., Dee, D., Hirahara, S. (2017): ERA5-Land: A new state-of-the-art Global Land Surface Reanalysis Dataset, 31st Conference on Hydrology - 2017 AMS annual meeting, Seattle, US

Muñoz Sabater, J., Dutra, E., Agustí-Panareda, A., Albergel, C., Arduini, G., Balsamo, G., Boussetta, S., Choulga, M., Harrigan, S., Hersbach, H., Martens, B., Miralles, D. G., Piles, M., Rodrí guez Fernández, N. J., Zsoter, E., Buontempo, C., Thépaut, J. N. (2021): ERA5-Land: A state-ofthe-art global reanalysis dataset for land applications, Earth Syst. Sci. Data Discuss. [preprint], in review

Myneni, R., Knyazikhin, Y., Park, T. (2015): MCD15A3H MODIS/Terra+Aqua Leaf Area Index/FPAR 4-day L4 Global 500m SIN Grid V006 [Data set], NASA EOSDIS Land Processes DAAC, verfügbar unter: https://doi.org/10 5067/MODIS/MCD15A3H.006 (letzter Zugriff: 12.03.2020)

NASAa (2021): National Aeronautics and Space Administration (NASA), Landsat Science, verfügbar unter: https://landsat.gsfc.nasa.gov/ (letzter Zugriff: 22.02.2021)

NASAb (2021): National Aeronautics and Space Administration (NASA), MODIS Mission, verfügbar unter: https://modis.gsfc.nasa.gov/ (letzter Zugriff: 22.02.2021)

Nearing, G. S., Kratzert, F., Sampson, A. K., Pelissier, C. S., Klotz, D., Frame, J. M., Prieto, C. Gupta, H. V. (2020): What Role Does Hydrological Science Play in the Age of Machine Learning? Water Resour. Res., e2020WR028091, https://doi. org/10.1029/2020wr028091

Newman, A. J., Clark, M. P., Sampson, K., Wood A., Hay, L. E., Bock, A., Viger, R. J., Blodgett, D., Brekke, L., Arnold, J. R., Hopson, T., Duan, Q. (2015): Development of a large-sample watershed-scale hydrometeorological data set for the contiguous USA: data set characteristics and assessment of regional variability in hydrologic model performance, Hydrol. Earth Syst. Sci., 19, S. 209-223, https://doi.org/10.5194/hess-19209-2015

Oerlemans, J., Anderson, B., Hubbard, A. Huybrechts, P., Johannesson, T., Knap, W. H., Schmeits, M., Stroeven, A. P., van de Wal, R S. W., Wallinga, J. (1998): Modelling the response of glaciers to climate warming, Clim. Dynam., 14, S. 267-274, https://doi.org/10.1007/ s003820050222

Olden, J. D., Poff, N. L. (2003): Redundancy and the choice of hydrologic indices for characterizing streamflow regimes, River Res. Appl., 19, S. 101-121, https://doi.org/10.1002/rra.700 Panagos, P. (2006): The European soil database GEO: connexion, 5(7), S. 32-33

Panagos, P., Van Liedekerke, M., Jones, A. Montanarella L. (2012): European Soil Data Centre: Response to European policy support and public data requirements, Land Use Policy, 29(2), S. 329-338, https://doi.org/10.1016/j. landusepol.2011.07.003

Pelletier, J. D., Broxton, P. D., Hazenberg, P. Zeng, X., Troch, P. A., Niu, G., Williams, Z. C. Brunke, M.A., Gochis, D. (2016): Global 1-km Gridded Thickness of Soil, Regolith, and Sedimentary Deposit Layers. ORNL DAAC, Oak Ridge, Tennessee, USA, https://doi.org/10.3334/ ORNLDAAC/1304

Prohaska, S., Brilly, M., Kryžanowski, A. (2020): Cooperation of hydrologists from the Danube River Basin, Hydrol. Earth Syst. Sci. Discuss., S. 1-10, https://doi.org/10.5194/hess-2020-66 Python Software Foundation (2020): Python Language Reference, verfügbar unter https:// www.python.org. (letzter Zugriff: 17.05.2021)
QGIS Development Team (2020): QGIS Geographic Information System, Open Source Geospatial Foundation Project, verfügbar unter: http:// qgis.org. (letzter Zugriff: 17.05.2021)

R Core Team (2020): R: A language and environment for statistical computing, R Foundation for Statistical Computing, Wien, Österreich, verfügbar unter: https://www.r-project.org. (letzter Zugriff: 17.05.2021)

Sankarasubramanian, A., Vogel, R. M., Limbrunner, J. F. (2001): Climate elasticity of streamflow in the United States, Water Resour Res., 37, S. 1771-1781, https://doi.org/10.1029/ 2000WR900330

Sawicz, K., Wagener, T., Sivapalan, M., Troch, P A., Carrillo, G. (2011): Catchment classification: empirical analysis of hydrologic similarity based on catchment function in the eastern USA, Hydrol. Earth Syst. Sci., 15, S. 2895-2911, https:// doi.org/10.5194/hess-15-2895-2011

Schaake, J. C., Hamill, T. M., Buizza, R., Clark M. (2007): HEPEX: the hydrological ensemble prediction experiment, Bulletin of the American Meteorological Society, 88(10), S. 1541-1548, https://doi.org/10.1175/BAMS-88-10-1541

Schumm, S. A. (1956): Evolution of drainage systems and slopes in Badlands at Perth Amboy, New Jersey, GSA Bulletin, 67(5), S. 597-646, https://doi.org/10.1130/0016-7606(1956)67 [597:EODSAS]2.0.CO;2

Singh, R., Archfield, S. A., Wagener, T. (2014): Identifying dominant controls on hydrologic parameter transfer from gauged to ungauged catchments-A comparative hydrology approach, J. Hydrol., 517, S. 985-996, https://doi. org/10.1016/j.jhydrol.2014.06.030

Sit, M., Demiray, B. Z., Xiang, Z., Ewing, G. J., Sermet, Y., Demir, I. (2020): A comprehensive review of deep learning applications in hydrology and water resources, Water Sci. Technol. 82(12), S. 2635-2670, https://doi.org/10.2166/ wst.2020.369

Smith, M. B., Seo, D. J., Koren, V. I., Reed, S. M., Zhang, Z, Duan, Q, Moreda, E, Cong, S. (2004): The Distributed Model Intercomparison Project (DMIP): motivation and experiment design, J. Hydrol., 298, S. 4-26, https://doi.org/10.1016/j. jhydrol.2004.03.040

Tallaksen, L., Van Lanen, H. A. J. (Eds.) (2004); Hydrological drought. Processes and estimation methods for streamflow and groundwater, Developments in Water Science, 48, Elsevier Science B.V., Amsterdam, ISBN: 978-0444516886, 579pp Toth, B., Weynants, M., Nemes, A., Makó, A. Bilas, G., Toth, G. (2015): New generation of hydraulic pedotransfer functions for Europe, European Journal of Soil Science, 66, S. 226-238, https://doi.org/10.1111/ejss.12192

Toth, B., Weynants, M., Pasztor, L., Hengl, T. (2017): 3D soil hydraulic database of Europe at $250 \mathrm{~m}$ resolution, Hydrological Processes, 31, S. 2662-2666, https://doi.org/10.1002/hyp. 11203

Trabucco, A., Zomer, R. (2019): Global Aridity Index and Potential Evapotranspiration (ET0) Climate Database v2, CGIAR Consortium for Spatial Information (CGIAR-CSI), published online, verfügbar unter: https://doi.org/ 10.6084/m9.figshare.7504448.v3 (letzter Zugriff: 18.03.2020)

Van Lanen, H. A. J., Wanders, N., Tallaksen, L. M., Van Loon, A. F. (2013): Hydrological drought across the world: impact of climate and physical catchment structure, Hydrol. Earth Syst. Sci., 17, S. 1715-1732, https://doi.org/10.5194/hess17-1715-2013

Vermote, E. (2015): MOD09Q1 MODIS/Terra Surface Reflectance 8-Day L3 Global 250m SIN Grid V006 [Data set], NASA EOSDIS Land Processes DAAC, verfügbar unter: https://doi.org/ 10.5067/MODIS/MOD09Q1.006 (letzter Zugriff: 12.03.2020) 
Warszawski, L., Frieler, K., Huber, V., Piontek, F., Serdeczny, O., Schewe, J. (2014): The intersectoral impact model intercomparison project (ISI-MIP): project framework, Proceedings of the National Academy of Sciences, 111(9), S. 3228-3232, https://doi.org/10.1073/pnas. 1312330110

Westerberg, I. K., McMillan, H. K. (2015): Uncertainty in hydrological signatures, Hydrol. Earth Syst. Sci., 19, S. 3951-3968, https://doi. org/10.5194/hess-19-3951-2015

Westerberg, I. K., Wagener, T., Coxon, G., McMillan, H. K., Castellarin, A., Montanari, A. Freer, J. (2016): Uncertainty in hydrological signatures for gauged and ungauged catchments, Water Resour. Res., 52, S. 1847-1865, https://doi. org/10.1002/2015WR017635
WGMS (World Glacier Monitoring Service) (2005): Glacier Mass Balance Bulletin, No. 8 (2002-2003), edited by: Haeberli, W., Noetzli, J., Zemp, M., Baumann, S., Frauenfelder, R., Hoelzle, M., Department für Geographie, Universität Zürich, 100pp

Woods, R. A. (2009): Analytical model of seasonal climate impacts on snow hydrology: Continuous snowpacks, Adv. Water Resour. 32, S. 1465-1481, https://doi.org/10.1016/j. advwatres.2009.06.011

Yang, X., Giusti, M. (2020): ERA5-Land: data documentation, CDS dataset documentation, European Centre for Medium-Range Weather Forecasts (ECMWF), verfügbar un ter: https://confluence.ecmwf.int/display/CKB/
ERA5-Land\%3A+data+documentation (letzter Zugriff: 30.11.2020)

Yokoo, Y., Sivapalan, M. (2011): Towards reconstruction of the flow duration curve: development of a conceptual framework with a physical basis, Hydrol. Earth Syst. Sci., 15, S. 2805-2819, https://doi.org/10.5194/hess-15-2805-2011

Hinweis des Verlags Der Verlag bleibt in Hinblick auf geografische Zuordnungen und Gebietsbezeichnungen in veröffentlichten Karten und Institutsadressen neutral. 